\title{
Exploiting [2+2] cycloaddition chemistry: achievements with allenes
}

\author{
Benito Alcaide, ${ }^{* a}$ Pedro Almendros ${ }^{* b}$ and Cristina Aragoncillo $* a$
}

Received 9th July 2009

First published as an Advance Article on the web 22nd October 2009

DOI: $10.1039 / b 913749$ a

The allene moiety represents an excellent partner for the [2+2] cycloaddition with alkenes and alkynes, affording the cyclobutane and cyclobutene skeletons in a single step. This strategy has been widely studied under thermal, photochemical and microwave induced conditions. More recently, the use of transition metal catalysis has been introduced as an alternative relying on the activation of the allenic component. On the other hand, the intramolecular version has attracted much attention as a strategy for the synthesis of polycyclic compounds in a regio- and stereoselective fashion. This critical review focuses on the most recently developed [2+2] cycloadditions on allenes along with remarkable early works accounting for the mechanism, the regio- and diastereoselectivity of the cycloadducts formed (103 references).

\section{Introduction}

During the last few years, the chemistry of allenes has been extensively studied and many reviews on their preparation and reactivities have been published. ${ }^{1}$ They have shown an interesting reactivity and selectivity affording complex structures in a limited number of steps. ${ }^{2}$ In fact, the chemistry of allenes has been applied for the preparation of natural and non-natural products of interest. In particular, the $[2+2]$

\footnotetext{
a Departamento de Química Orgánica I, Facultad de Química, Universidad Complutense de Madrid, 28040 Madrid, Spain. E-mail: alcaideb@quim.ucm.es, caragoncillo@quim.ucm.es; Fax: (+34) 91-3944103; Tel: (+34) 91-3944314, (+34) 91-3945169

${ }^{b}$ Instituto de Química Orgánica General. Consejo Superior de Investigaciones Cientificas, CSIC. Juan de la Cierva, 3, 28006 Madrid, Spain.E-mail: Palmendros@iqog.csic.es, Fax: (+34) 91-5644853; Tel: (+34) 91-5618806
}

cycloaddition of allenes represents an important strategy for the preparation of cyclobutane derivatives with high atom economy. The importance of the cyclobutane containing compounds both as target molecules as well as useful building blocks for the construction of more complex structures has been widely demonstrated. ${ }^{3}$ In particular, due to the inherent ring strain, cyclobutanes can undergo fragmentation and ring-expansion reactions for the synthesis of both acyclic and cyclic systems. ${ }^{4} \mathrm{~A}$ high number of strategies has been published, showing the reactivity of these substrates. ${ }^{5}$

Although the thermal process is forbidden by the Woodward-Hoffmann rules, ${ }^{6}$ and the Fukui's frontier orbital theory, ${ }^{7}$ the $[2+2]$ cycloaddition of allenes has been studied photochemically, under thermal conditions involving diradical intermediates, and more recently by the use of transition metal catalysts. It is of special interest when the [2+2] cycloaddition is carried out in an intramolecular fashion for the construction

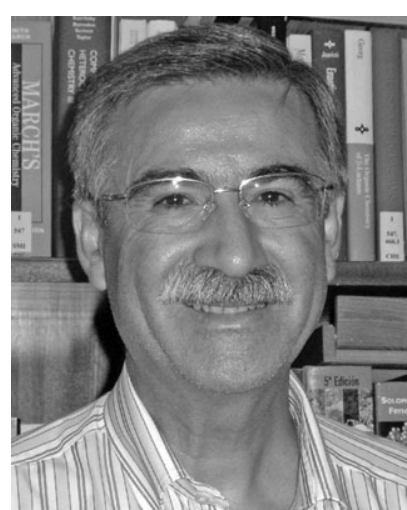

Benito Alcaide
Benito Alcaide was born in Aldea del Rey, Ciudad Real, Spain, in 1950. He received his BS degree (1972) and $P h D$ degree (1978) from the Universidad Complutense de Madrid (UCM) under the supervision of Professor Franco Fernández. After a 4 year postdoctoral period working on the chemistry of $\alpha$-iminoketones and related compounds, he began working on $\beta$-lactam chemistry. In 1984 he assumed a position of Associate Professor of Organic Chemistry and in 1990 was promoted to Full Professor at the UCM. His current recent interests include $\beta$-lactam chemistry, asymmetric synthesis of compounds of biological interest, allenes, metal-promoted cyclizations, and organocatalysis.

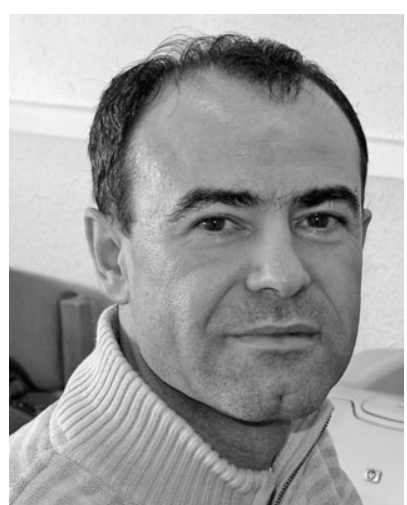

Pedro Almendros
Pedro Almendros was born in Albacete (Spain) in 1966. He received his $B S$ degree (1989) and his PhD degree (1994) with Profs. Molina and Fresneda (Universidad de Murcia). After three years postdoctoral stay with Prof. Thomas at the University of Manchester, with a Spanish MEC Postdoctoral Fellowship (1995-1997) and an European Marie Curie Postdoctoral Grant (1997-1998), he joined the research group of Prof. Benito Alcaide (UCM, Madrid) in 1998 as Associate Researcher. Subsequent appointments have included Assistant Professor at the UCM (2000-2002), and Cientifico Titular at the IQOG, CSIC, Madrid. In 2007 he was promoted to Investigador Cientifico. His research interests include $\beta$-lactams, allenes, and heterocyclizations. 
of polycyclic structures in one step, thus bringing many benefits.

In this contribution, we wish to review the most recent advances on the $[2+2]$ cycloaddition chemistry of allenes, but also take into account early works, concerning mechanistic studies, as a base for a better understanding. Most of the examples are thermal [2+2] cycloadditions, however, recently an increased interest in the study of the $[2+2]$ cycloaddition promoted by metal catalysts has been observed.

\section{Thermal $[2+2]$ cycloadditions}

Thermal $[2+2]$ cycloaddition of allenes has been studied in depth in a wide variety of allenic systems, and many examples have been reported in literature. In general, the $[2+2]$ cycloaddition of allenes requires high temperatures (over $200{ }^{\circ} \mathrm{C}$ ) and long reaction times, suffering sometimes low regio- and stereoselectivity on the observed compounds. On the other hand, because the thermal process is not allowed by the Woodward-Hoffmann rules ${ }^{6}$ and the Fukui's frontier orbital theory, ${ }^{7}$ the mechanistic details associated with this process have constituted an important topic of study. In fact, although some authors have reported the concertedness of the reaction by a contribution of the cumulated orthogonal $\pi$-bond of allene via a $\left[\left(\pi_{2 \mathrm{~s}}+\pi_{2 \mathrm{~s}}\right)_{\text {allene }}+\pi_{2 \mathrm{~s} \text { alkene }}\right]$ process, most of the examples have been explained via a stepwise diradical mechanism.

On the other hand, microwave irradiation has been employed as an unconventional energy source for a wide number of organic reactions and has been recognized as a powerful tool for the preparation of complex structures in short reaction times, with a minimization of decomposition and by-products with an improvement of stereo-, regio- or chemoselectivity instead of conventional thermal conditions. ${ }^{8}$

In the following section the mechanistic aspects of the dimerization and intermolecular [2+2] cycloaddition of allenes have been discussed. Literature from 1970 to 1992 , approximately, has provided an excellent base to understand the reactivity, regio- and stereoselectivity and the reversibility of the intermediates involved in the process. In addition, more

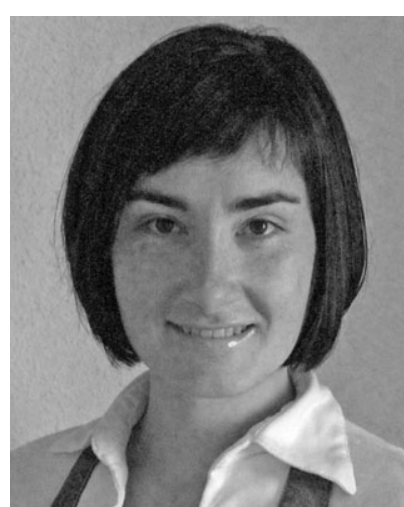

Cristina Aragoncillo
Cristina Aragoncillo was born in Madrid (Spain) in 1974. She obtained her BS degree (1997) and PhD degree (2002) from the UCM under the supervision of Profs Alcaide and Almendros. After 2 years as a Marie-Curie postdoctoral fellow at the University of Bristol working with Prof. Aggarwal, she came back to Madrid in May of 2005 at the IQOG, CSIC, with an I3P contract. Since January of 2006 she has been a Ramón y Cajal Researcher at the UCM in the research group of Prof. Alcaide. Her research is focused on $\beta$-lactam chemistry, asymmetric synthesis, allene chemistry, and metal-catalyzed coupling reactions. recent examples of the intermolecular version followed by intramolecular examples in allenenes and allenynes have been presented.

\subsection{Dimerization and intermolecular $[2+2]$ cycloadditions of allenes under thermal conditions}

Allene dimerization which results in the formation of substituted dimethylencyclobutanes has been studied intensely. ${ }^{9}$ When the allene is symmetrically substituted, two possible regioisomers are obtained (Scheme 1).

However, when the allene moiety is not symmetrically substituted, a mixture of several dienes can be formed: headto-head adduct $\mathbf{1}$, head-to-tail adduct $\mathbf{2}$ and tail-to-tail adducts $\mathbf{3}$ and $\mathbf{4}$, along with the corresponding cis-, trans-, $Z$ - and E-isomers (Scheme 2). ${ }^{10}$

The high stereoselectivity observed of some $[2+2]$ cycloadditions of allenic systems pointed toward the possibility that these reactions were concerted reactions, ${ }^{11}$ with allene acting in an antarafacial manner to the way that ketene has been demonstrated to behave. ${ }^{12}$ For example, it has been described that chloroallene and 1,1-dimethylallene dimerize predominantly in a head-to-head manner (Scheme 3). ${ }^{11}$

By contrast, 1,1-difluoroallene ${ }^{13}$ and 3,3-dimethyl-1cyanoallene ${ }^{14}$ dimerize in a head-to-tail manner. This results cannot be rationalized on the basis of a $\left[\pi_{2 s}+\pi_{2 s}\right]$ concerted process (Scheme 4). These experiments have shown the importance of the nature of the substituents in the allene moiety along with steric and electronic factors in the transition states.

Early studies on the secondary deuterium isotope effects of allene cycloadditions have indicated that $[2+2]$ dimerization
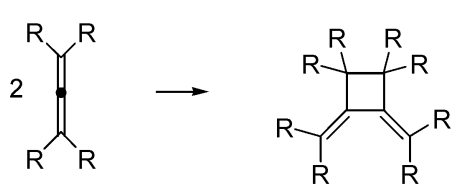

and/or

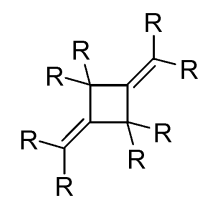

Scheme 1 Possible regioisomers observed in the dimerization of symmetrically substituted allenes through intermolecular $[2+2]$ cycloaddition reaction.<smiles>[R]C=C</smiles>

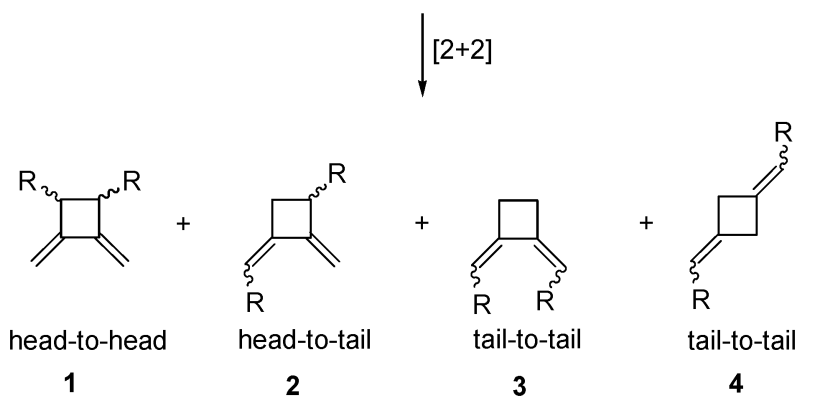

Scheme 2 Possible regioisomers observed in the dimerization of non-symmetrical-substituted allenes. 


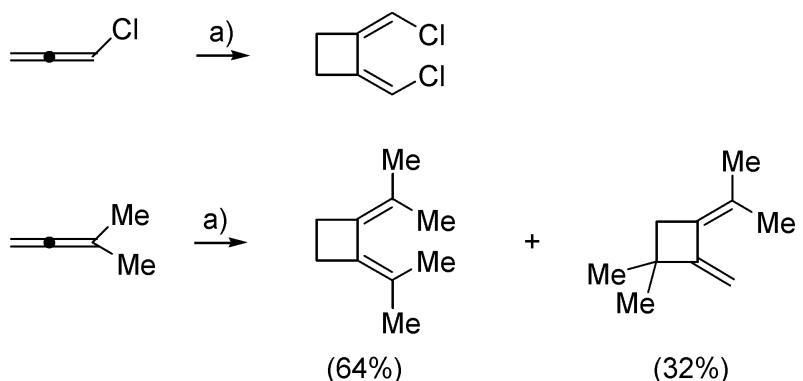

Scheme 3 [2+2] Dimerization of chloroallene and 1,1-dimethylallene. Reagents and conditions: (a) $\mathrm{Et}_{2} \mathrm{O}, 60{ }^{\circ} \mathrm{C}, 3-4$ days.

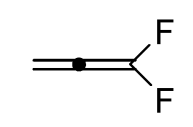

$\stackrel{\text { a) }}{\longrightarrow}$

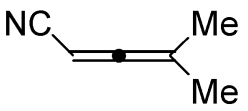

$\stackrel{\mathrm{b})}{\longrightarrow}$

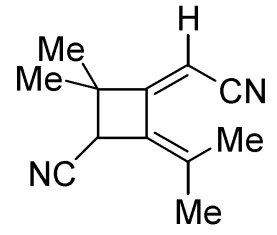

$(70 \%)$
Scheme 4 [2+2] Dimerization of difluoroallene and 3,3-dimethyl-1cyanoallene. Reagents and conditions: (a) $250-295{ }^{\circ} \mathrm{C}$. (b) $100{ }^{\circ} \mathrm{C}, 45$ $\min , \mathrm{N}_{2}$.

of allenes is not a synchronous process. ${ }^{15}$ In this context, the intermediacy of a perpendicular or freely rotating diradical 5 has been proposed in the thermal dimerization of 6 (Scheme 5). However, further proof of the stereospecificity of allene cycloaddition, ${ }^{16}$ which together with a striking preference for the more sterically crowded products (dimers having the exocyclic substituents in the hindered inward positions, tail-to-tail dimer 3 in Scheme 2) observed in the dimerization of substituted allenes, needed the development of a concerted two-step mechanism for allene dimerization. ${ }^{17}$

In this view, a bisallyl diradical intermediate either retains its stereochemical integrity throught its existence (presumably via a secondary orbital overlap), or it is formed from allene and close products in separate but individually stereospecific steps (presumably under steric and orbital symmetry control). MO calculations on $\mathbf{5}$ and its interconversion with its various stable valence isomers have demonstrated that $\mathbf{5}$ appears to

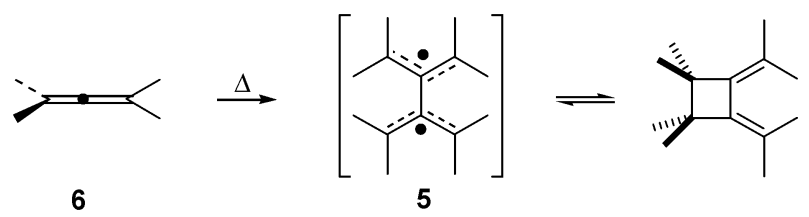

Scheme 5 Formation of a freely rotating diradical 5 in the thermal dimerization of $\mathbf{6}$. have no constraining electronic preference as to either molecular geometry or $\pi$ orbital symmetry. ${ }^{18}$

The question of whether a bisallyl diradical $\mathbf{5}$ is necessarily an intermediate in allene dimerization and, if it is an orthogonal or freely rotating specie has proven difficult to answer. Kiefer has reported the thermal and photochemical generation of 2,2'-bis(1,1-dimethylallyl) diradical from an azocyclane precursor. ${ }^{19}$ From the results obtained, the authors have suggested that intermediate $\mathbf{5}$ has a small but significant energy minimum at or near the perpendicular $\left(D_{2 \mathrm{~d}}\right)$ geometry, rather than a freely rotating $2-2^{\prime}$ bond. This is in line with expectations based on Hückel calculations ${ }^{18}$ and terminology, which describe $\mathbf{5}$ as "effectively orthogonal". ${ }^{20}$

Thus, the regio- and stereoselectivity observed in thermal dimerization of allenes has been interpreted on the basis of bis-allyl-diradical intermediates type 5, although a concerted pathway $\left[\left(\pi_{2 \mathrm{~s}}+\pi_{2 \mathrm{~s}}\right)_{\text {allene }}+\pi_{2 \mathrm{~s} \text { alkene }}\right.$ explains the results in many instances. ${ }^{21}$

Aproach of two molecules of allene takes place in a crossed configuration, followed by bond formation between the central $s p$ carbons. Simultaneous conrotatory twisting generates a perpendicular 2,2'-bisallyl diradical 5, which after rotation and disrotatory closure leads to the corresponding dimer 7 (Scheme 6).

On the other hand, considerable attention has been devoted to the study of the cycloaddition reactions of allenes with alkenes and alkynes. For example, the [2+2] cycloaddition of 1,1-dimethylallene with dimethylfumarate has afforded two cycloadducts 8 and 9 in a 11.5:1 ratio in which $>99 \%$ stereoselectivity is claimed (Scheme 7). ${ }^{22}$ The reaction of optically active 2,3-pentadiene with acrylonitrile is reported to give four stereoisomeric adducts, all of which are optically active. ${ }^{23}$ Despite these seemingly stereochemically concordant results, different mechanisms have been proposed: in the former, a concerted $\left[\pi_{2 s}+\pi_{2 s}\right]$ process was proposed; in the latter, a two-step, diradical intermediate mechanism.

In order to get a better understanding of the mechanistic details of the $[2+2]$ cycloaddition reaction of allenes with alkenes and alkynes, Pasto has carried out several studies involving the relative reactivity of different substituted allenes with alkenes and alkynes.

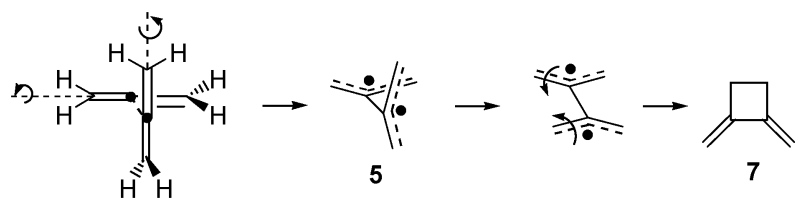

Scheme 6 Mechanistic proposal for the dimerization of allenes by $[2+2]$ cycloaddition.

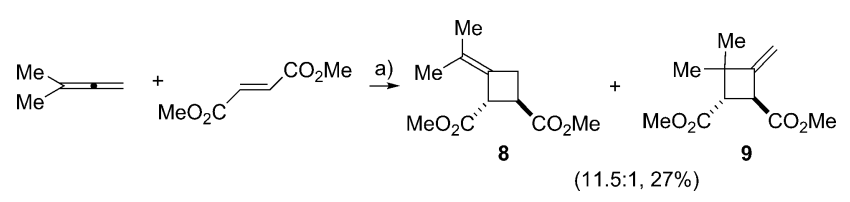

Scheme 7 [2+2] Cycloaddition of 1,1-dimethylallene with dimethylfumarate. Reagents and conditions: (a) $160-180{ }^{\circ} \mathrm{C}, 18-70 \mathrm{~h}$. 

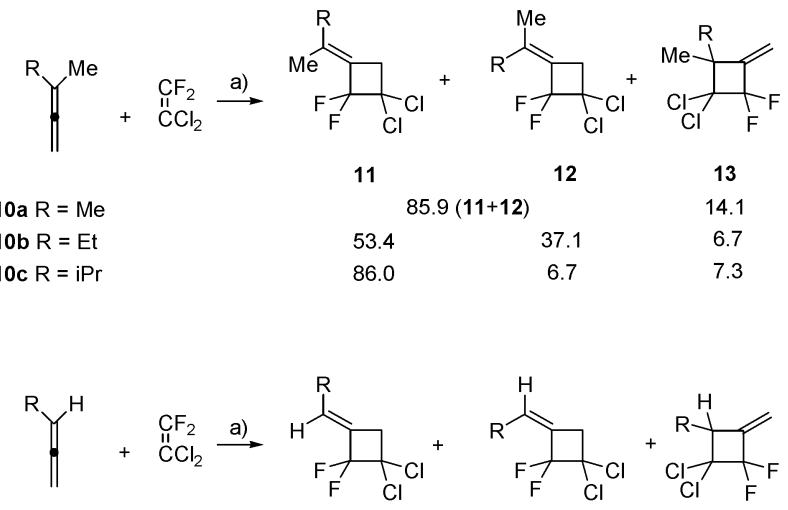

$\begin{array}{lccc} & 11 & 12 & 14 \\ 10 \mathrm{~d} R=\mathrm{Et} & 50.5 & 27.3 & 15.4 \\ 10 \mathrm{e} R=\mathrm{iBu} & 48.2 & 27.2 & 24.7 \\ 10 \mathrm{f} R=\mathrm{iPr} & 55.0 & 20.0 & 25.0 \\ 10 \mathrm{~g} R=\mathrm{tBu} & 55.0 & - & 35.3\end{array}$

Scheme 8 [2+2] Cycloaddition of 1,1-di- and monosubstituted allenes 10a-g with 1,1-dichloro-2,2-difluoroethene. Reagents and conditions: (a) $160{ }^{\circ} \mathrm{C}, 1-2$ days.

First, this author has studied the cycloaddition of substituted allenes with 1,1-dichloro-2,2-difluoroethene as a model for the two-step diradical-intermediate $[2+2]$ cycloaddition of allenes. ${ }^{24}$ Thus, the stereoselectivities, chemoselectivities, relative reactivities and kinetic isotope effects in the cycloaddition reaction of substituted allenes with 1,1-dichloro-2,2-difluoroethene, have been determined by comparison with the radicalchain addition of benzenethiol to substituted allenes. ${ }^{25}$

The cycloaddition reaction of 1,1-di- and monosubstituted allenes 10a-f with 1,1-dichloro-2,2-difluoroethene has been carried out at $160{ }^{\circ} \mathrm{C}$ in a sealed tube affording mixtures of the adducts 11-14 (Scheme 8).

The formation of the $[2+2]$ cycloaddition products has been rationalized in terms of the mechanism shown in Scheme 9. The formation of the stereoisomeric adducts $\mathbf{1 1}$ and $\mathbf{1 2}$ requires the formation of the two stereoisomeric diradical intermediates $\mathbf{1 5}$ and 16, the former undergoing ring closure to form only $\mathbf{1 1}$ and $\mathbf{1 4}$ and the latter to form only $\mathbf{1 2}$ and $\mathbf{1 4 .}$

A priori, radical formation should be more favored at the most substituted terminal carbon atom of the allene

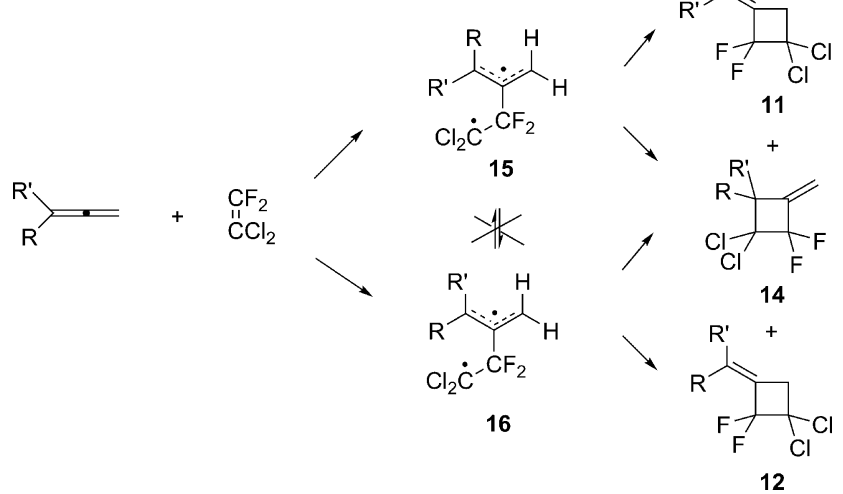

Scheme 9 Mechanistic proposal to explain the formation of compounds 11, 12 and 14 via diradical intermediates 15 and $16 .{ }^{24}$

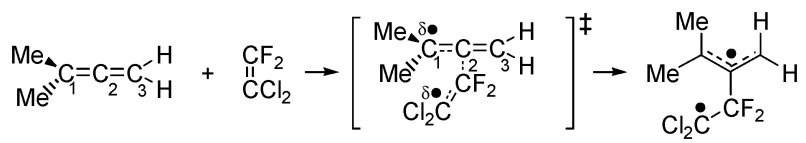

Scheme 10 Formation of diradical intermediate by attack of the most highly substituted double bond.

chromophore. This requires attack on the $2 \mathrm{p}$ AO on $\mathrm{C} 2$ of the most highly substituted double bond with rotation of the developing radical center to form the planar allyl radical portion of the intermediate, as it is illustrated in Scheme 10. In fact, the KIE's measured for the addition to 1,1-dimethylallene has confirmed this prediction. ${ }^{24}$

The stereoselectivity observed about the exocyclic double bond in the cycloadducts has been interpreted in terms of steric interactions generated in the transition states for formation of $\mathbf{1 5}$ and $\mathbf{1 6}$, the rate of formation of the latter decreasing with increasing size of $\mathrm{R}$. Thus, during the formation of $\mathbf{1 5}$ and 16, the rotational and translational motions illustrated in $\mathbf{1 5}$ and $\mathbf{1 6}$ in Scheme 11 must occur. In $\mathbf{1 7}$ the largest group $\mathrm{R}^{\prime}$ attached to $\mathrm{C} 1$ rotates away from the approaching reagent $\mathrm{X}=\mathrm{Y}$. This mode of rotation generates no adverse steric effects between $\mathrm{R}^{\prime}$ and the approaching reagent $\mathrm{X}=\mathrm{Y}$ but does result ultimately in the formation of the less thermodynamically endo orientation of $\mathrm{R}^{\prime}$ in the allyl radical. Rotation of the $\mathrm{R}^{\prime}$ group toward the approaching reagent $\mathrm{X}=\mathrm{Y}$ as in 18 generates an adverse steric interaction between $R^{\prime}$ and $\mathrm{X}=\mathrm{Y}$, which will increase as the size of $\mathrm{R}^{\prime}$ increases. This mode of rotation, however results in the formation of the most thermodynamically stable allyl radical. The experimental results have definitely indicated that the steric effects between $\mathrm{R}\left(\mathrm{R}^{\prime}\right)$ and the approaching reagent, generated on rotation of the $\mathrm{RCR}^{\prime}$ portion of the allene, dominate and that the extent of rotation is greater than that of translation in the transition state, suggestive of a rather early transition state.

The decrease in reactivity in the monoalkylallene and 1,1-dialkylallene series as the size of the $\mathrm{R}$ group increases has been explained due to a decrease in the rate of formation of diradical intermediate type 16. The increase in reactivity in the sequence monoalkyl- $<1,1$-dialkyl- $<1,1,3$-trialkylallenes is due to an incremental raising in the energies of the $\pi$
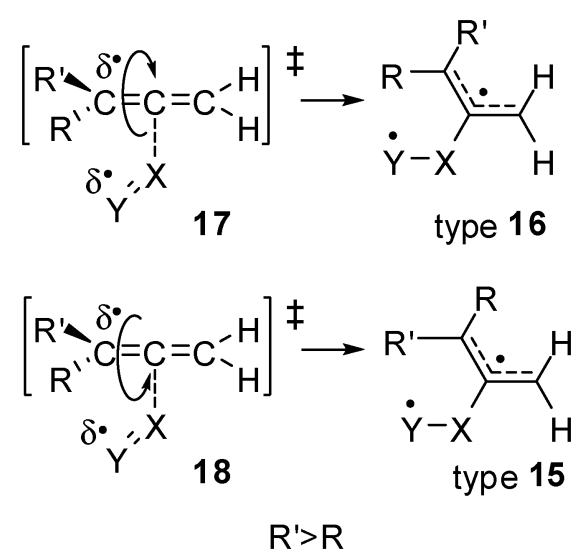

Scheme 11 Transition states proposed for formation of radicals type 15 and 16 
MO's in the sequence, which result in an increase in the rate of reaction.

The chemoselectivity has also been determined by steric interactions generated in the transition states for ring closure, favoring closure to the least substituted end of the allyl radical as the degree of substitution and size of the alkyl groups increase. Thus, the direction of ring closure of the diradical intermediate is dependent on the degree of substitution at the termini of the allyl radical portion of the diradical intermediate and on the size of the alkyl group(s) attached to the allyl radical. The diradical intermediates derived from the 1,1dialkylallenes show significantly greater tendency to ring close to the least substituted end of the allyl radical. This is undoubtedly due to the greater steric congestion encountered in the transition state for ring closure to the most highly substituted end. However, the data derived with monoalkylallenes have shown that as the size of the $\mathrm{R}$ group increases, ring closure to the most highly substituted end of the allyl radical increases. This steric effect seems to be in opposition to that causing the difference between the relative importance of the two modes of ring closure of the mono- and disubstituted systems.

The above criteria have been applied to the results derived from the cycloaddition reactions of substituted allenes with $N$-phenylmaleimide. ${ }^{26}$ In these experiments, four expected cycloadducts were obtained along with ene products. The chemoselectivities, stereospecificities, and relative reactivities have been explained via two-step diradical-intermediate processes.

Due to the nature of 1,1-dichloro-2,2-difluoroethene and $N$-phenylmaleimide, information on possible cleavage and internal rotation processes of the diradical intermediate was not accessible. In order to gain more detailed information on the competing reactions of diradical intermediates, reactions of substituted allenes with diethyl fumarate and maleate have been investigated. ${ }^{27}$

The reaction of diethyl fumarate (DEF) with 1,1-dimethylallene has afforded adducts 19-21, all containing the transdiester stereochemistry (Scheme 12). However, the reaction of dimethylallene with diethyl maleate (DEM) has afforded both cis- and trans-diester products 19-24. Interestingly, isomerization of diethyl maleate to diethylfumarate in the presence of 1,1dimethylallene has been observed.

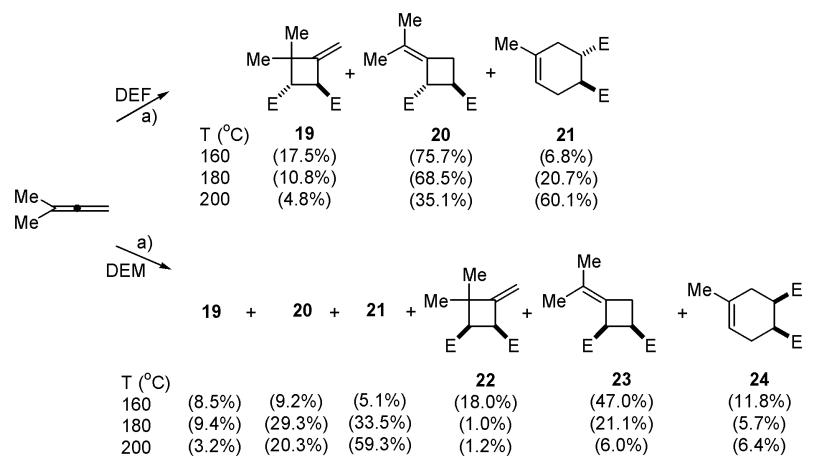

Scheme 12 [2+2] Cycloaddition of 1,1-dimethylallene with diethyl maleate and diethyl fumarate. Reagents and conditions: (a) 160, 180 or $200^{\circ} \mathrm{C}$, toluene, sealed tube.

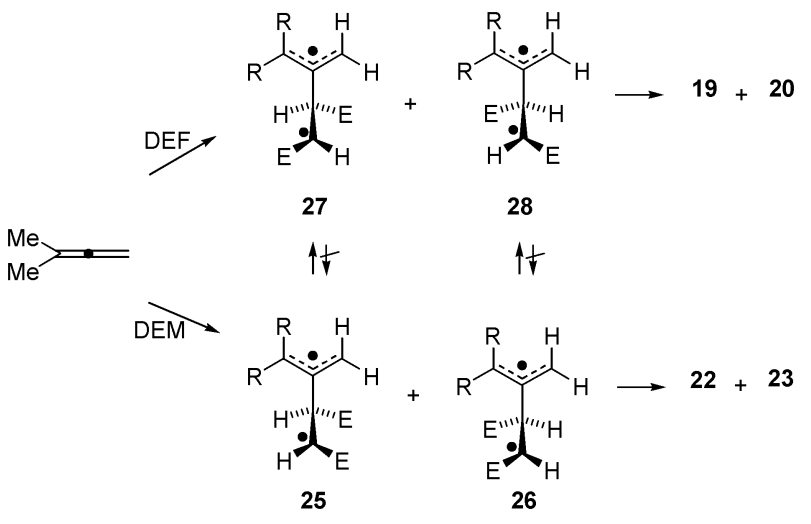

Scheme $\mathbf{1 3}$ Formation of diradicals $\mathbf{2 5 - 2 8}$ in the [2+2] cycloaddition of 1,1-dimethylallene with diethyl maleate and diethyl fumarate. ${ }^{27}$

The results have been rationalized in terms of a diradical mechanism in which internal rotation and cleavage reactions of the diradical intermediate compete with ring closure reactions. The results have been accounted as shown in Scheme 13 in which the diradical intermediates derived from dimethylallene and diethylmaleate (25 and 26) undergo internal rotation to generate the diradical intermediates derived from the reaction of 1,1-dimethylallene and DEF (27 and 28). However, the diradical intermediates derived from 1,1-dimethylallene and DEF do not undergo rotation to those derived from 1,1dimethylallene and DEM (Scheme 13).

In 27 and 28 the large ester functions are antiperiplanar, representing the lowest energy conformations. In $\mathbf{2 5}$ and $\mathbf{2 6}$ derived from DEM the ester groups must be initially eclipsed. Such conformations represent energy maxima, and must undergo rotational relaxation to form synclinal-type conformations or undergo further rotation to form $\mathbf{2 7}$ and $\mathbf{2 8}$. The trend towards identical 19:20 ratios from DEF and DEM at higher temperatures has suggested that a minimum-energy conformation close to the eclipsed conformations must exit, but the barriers to rotation and cleavage must be rather small, and more rotation and cleavage occur at higher temperatures with 25 and 26. In order to account for the observed product distribution in the reactions, slight modifications in the conformations 25-28 have been required. Slight rotations about the newly formed $\mathrm{C}-\mathrm{C}$ bond result in lower energy conformations in which either the $\mathrm{C}-\mathrm{H}(29$ and 30) or the $\mathrm{C}$-ester bond (31 and $\mathbf{3 2}$ ) is in the plane of the allyl radical. Of these conformations $\mathbf{2 9}$ and $\mathbf{3 0}$ should be of lower energy than 31 and 32, due to smaller 1,3-diaxial-type interactions and should be favored (Scheme 14). An analysis of the steric interactions in the transition states presented for diradicalintermediate formation has suggested that 27 should be favored over 28. Thus, cycloadduct $\mathbf{2 0}$ would be expected to be the dominant cycloadduct formed.

A similar analysis of the rotational processes in the diradical intermediates $\mathbf{2 5}$ and $\mathbf{2 6}$ formed in the reaction of 1,1-dimethylallene with DEM is shown in Scheme 15. In this Scheme only the more favored rotational relaxations of $\mathbf{2 5}$ and $\mathbf{2 6}$ to 33 and 34 have been shown, which undergo subsequent ring closure to form the cis-diester products 22 and $\mathbf{2 3}$. In $\mathbf{2 5}$ and $\mathbf{2 6}$ the ester functions are eclipsed or synclinal, which have much higher 


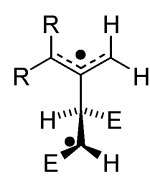

27

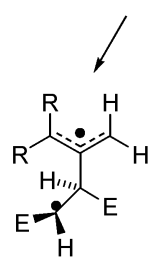<smiles>C1CCCC1</smiles><smiles>[R]C([R])=CC([CH])[C@H]([2H])[CH]</smiles>

31<smiles>[R]C1C(=C)[C@@H](F)[C@@H](F)C1[R]</smiles>

19

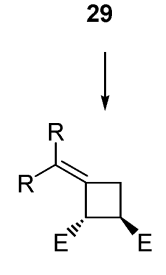

20<smiles>[R]C([R])=C([CH])[C@@H](C(C)C)C(F)(F)F</smiles>

28

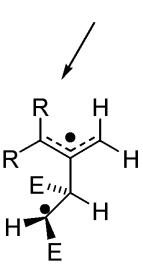

30<smiles>[R]C1C(=C)[C@@H](F)[C@@H]1CC</smiles>

19

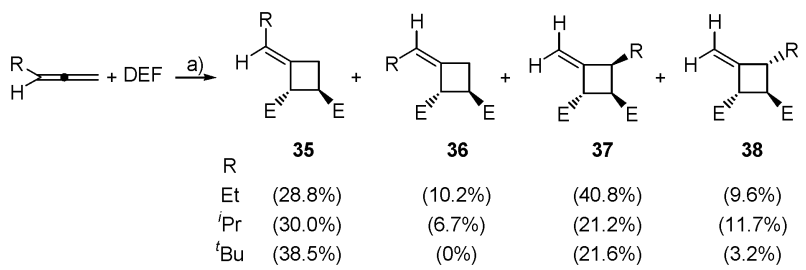

Scheme 16 [2+2] Cycloaddition reaction of monoalkylallenes with DEF. Reagents and conditions: (a) DEF as solvent, $160{ }^{\circ} \mathrm{C}$, 10-12 days.

Therefore, for the reactions of 1,1-dimethylallene with DEM, higher-energy diradical intermediates are formed compared to those from the reaction of 1,1-dimethylallene with DEF. The product distribution suggests that the energy barriers to ring closure are approximately the same in the two reactions but that the energy barrier to rotation is significantly reduced in the eclipsed or synclinal diradicals allowing internal rotation to compete effectively with ring closure. It also appears that the energy barrier for cleavage is similarly affected.

In contrast, the reaction of monoalkylallenes with DEF has provided a mixture of trans-diester adducts 35-38 (Scheme 16). ${ }^{27}$ It has been indicated that the yield of $\mathbf{3 6}$ relative to $\mathbf{3 5}$ decreases as the size of the alkyl group increases, suggesting a two-step, diradical mechanism. Trans, cis-cycloadduct $\mathbf{3 7}$ is formed predominantly over trans, trans-adduct $\mathbf{3 8}$ when $\mathrm{R}$ is tert-butyl.

Models have indicated that the transition states for formation of $\mathbf{3 5}$ would involve diradical intermediates $\mathbf{3 9}$ and $\mathbf{4 0}$ (Scheme 17). Intermediate 39 will undergo predominant rotational relaxation to form $\mathbf{4 1}$ which undergoes ring closure to form 35. The minor rotational pathway from 39 will form 42 which closes to afford the minor trans,trans-adduct 38. Intermediate $\mathbf{4 0}$ will undergo predominant rotational relaxation

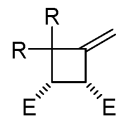

22

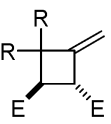

19
Scheme 15 Diradical conformations proposed for the formation of compounds 19, 20, 22 and 23 during the [2+2] cycloaddition of dimethylallene with diethyl maleate. ${ }^{27}$

energy conformations than those in which the ester functions are antiperiplanar or anticlinal. Thus, the rotation barriers from 33 to 29 , and 34 to 30 must be relatively small, providing for facile conformation isomerization. The substantially lower 19:20 product ratio observed in the reaction of 1,1-dimethylallene with DEM, compared to that observed in the reaction of 1,1-dimethylallene with DEF, have indicated that 34 undergoes rotation to form $\mathbf{3 0}$ in a lower extension than $\mathbf{3 3}$ does to form 29.
39

38
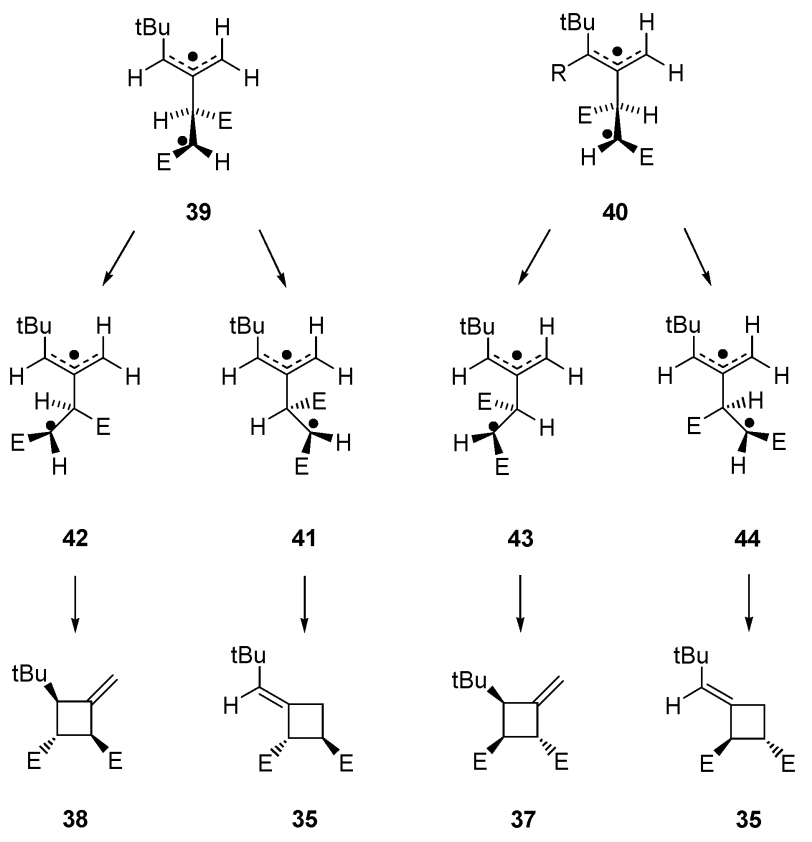

Scheme 17 Diradical conformations proposed for the formation of compounds 35, 37 and $\mathbf{3 8}$ during the [2+2] cycloaddition of monoalkylallene with diethyl fumarate. ${ }^{27}$ 
to form 43. If ring closure occurs faster than any other rotational process in $\mathbf{4 3}$, ring closure from $\mathbf{4 3}$ will form only the trans,cis-adduct 37 . The minor rotational pathway in $\mathbf{4 0}$ will produce $\mathbf{4 4}$ and ultimately $\mathbf{3 5}$. Thus, the conformational preferences in the diradical intermediates with antiperiplanar or anticlinal ester functions determine the product distributions.

Another studied feature of the [2+2] cycloaddition process has been the reversibility of formation of the diradical intermediates. Initially, the reversibility of the cycloaddition has been indicated by the cis to trans isomerization of the substituted allene or from the stereochemistry about the exocyclic double bonds in the cycloadducts. Due to the limited number of substituted alkenes in which cis to trans isomerization of the alkene can be monitored, the use of chiral allenes has solved this problem, thus the reversibility of formation of the diradical intermediates being indicated by the loss of enantiomeric excess (ee) of the starting allene and a decrease in the ee of the cycloadducts with extent of reaction. ${ }^{28}$ The results derived from the cycloaddition reaction of chiral allenes have provided stereochemical information on the formation of the diradical intermediates and on their ring closure. First, the stereochemical features of the [2+2] cycloaddition reaction of enantioenriched 1,3-dimethylallene and acrylonitrile affording cycloadducts 45-48 have been studied (Scheme 18). Two separate experiments have been carried out starting from 1,3-dimethylallene in $30.3 \%$ ee and recovering it in $28.3 \%$ $e e$; and secondly by using 1,3-dimethylallene in $28.3 \%$ ee and recovering it in $25 \% e e$. The ee's of the starting and recovered 1,3-dimethylallene have suggested that formation of diradical intermediates is irreversible, or if the intermediates are formed reversibly, there is essentially complete chirality transfer of 1,3-dimethylallene to the intermediates.

Molecular mechanics calculations on the possible orientations of approach of the acrylonitrile to $(S)-(+)-1,3$-dimethylallene have indicated that the lower energy orientation is the one in which acrylonitrile is oriented in the upward direction (diastereomers 49 and 50), being $\mathbf{4 9}$ of lower energy than $\mathbf{5 0}$ (Scheme 19). Scheme 19 shows the corresponding diradicals 51-54 derived from the approaching of 1,3-dimethylallene to acrylonitrile. Ring closure of diradicals 51-54 at the right or the left end of the allyl radical will produce cyclobutanes 45-48.

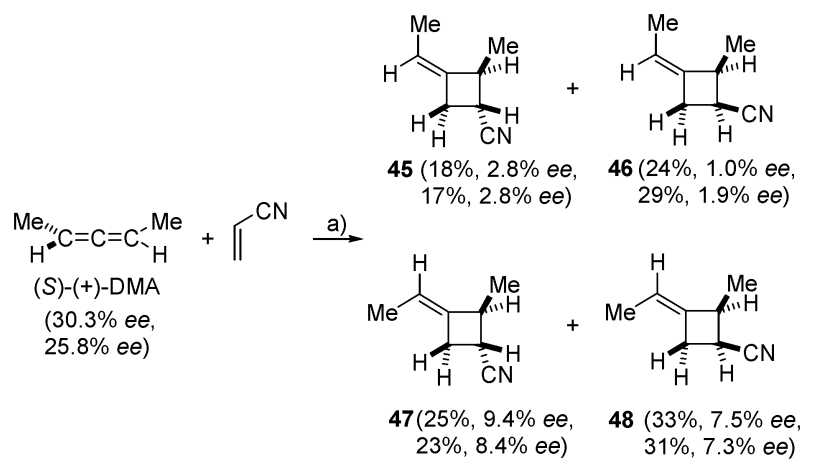

Scheme 18 [2+2] Cycloaddition of $(S)-(+)-1,3$-dimethylallene with acrylonitrile. (a) Reagents and conditions: (a) toluene- $\mathrm{d}_{8}$, hydroquinone, $148^{\circ} \mathrm{C}, 5$ days.
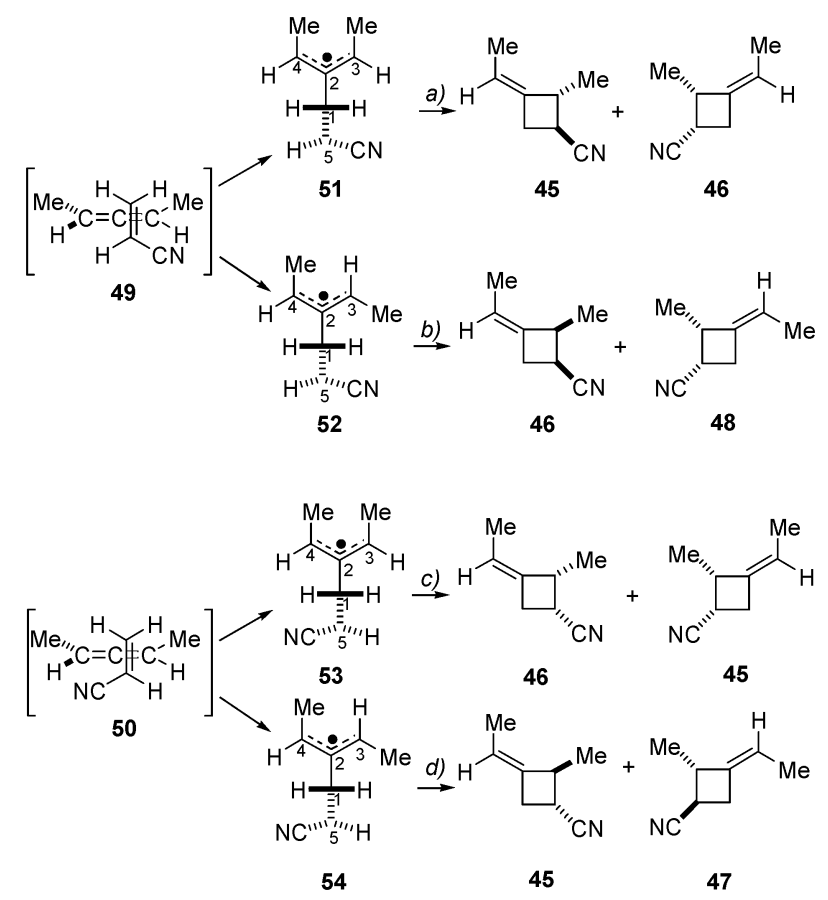

Scheme 19 Lower energy possible orientations of approach of $(S)-(+)$-1,3-dimethylallene to acrylonitrile. ${ }^{28}$

Cycloadducts $\mathbf{4 5}$ and $\mathbf{4 6}$ can be formed via three different channels; for $\mathbf{4 5}$, channels $a, c$ and $d$, and for $\mathbf{4 6}$, channels $a, b$ and $c$. In both of these two sets of reaction channels, two ringclosed products possessing the $S$ configuration at $\mathrm{C} 2$ and one having the $R$ configuration at $\mathrm{C} 2$ may be formed. The latter channels result in reduction of the ee transferred to the cycloadducts $\mathbf{4 5}$ and $\mathbf{4 6}$. In addition, the energy barriers for racemization of $\mathbf{5 1}$ and $\mathbf{5 3}$ appear to be low enough to allow for some competitive racemization, further reducing the $e e^{\prime} s$ retained in $\mathbf{4 5}$ and $\mathbf{4 6}$. The observed ee's of $\mathbf{4 5}$ and $\mathbf{4 6}$ are in all probability derived from ring closure of the diradical intermediates $\mathbf{5 2}$ and $\mathbf{5 4}$, which are of higher intrinsic asymmetry and are not capable of undergoing racemization competitive with ring closure.

In contrast, cycloadducts $\mathbf{4 7}$ and $\mathbf{4 8}$ are formed only via single reaction channels ( $d$ and $b$, respectively), both leading to the $S$ configuration at $\mathrm{C} 2$ in the cycloadducts. This, along with the expectation that racemization of $\mathbf{5 2}$ and $\mathbf{5 4}$ will not be competitive with ring closure will result in higher transfers of the $e e$ of the 1,3-dimethylallene to these products. The fact that only 37 and $29 \%$ of the ee of the starting 1,3-dimethylallene has been transferred to the cycloadducts, along with the fact that the recovered unreacted 1,3-dimethylallene has not lose any ee during the course of the reaction, has implied that the diradical intermediates 51-54 are formed irreversibly.

On the other hand, [2+2] cycloaddition of $(S)-(+)-1,3-$ dimethylallene with methyl acrylate has produced the four cycloadducts $\mathbf{5 5}-\mathbf{5 8}$ (Scheme 20). ${ }^{28}$

It has been observed that there is no loss in the ee of the 1,3dimethylallene during the course of the cycloaddition reaction, which, in view of the fact that derived cycloadducts possess lesser ee's than the starting 1,3-dimethylallene, indicates that the formation of the diradical intermediates is irreversible. 


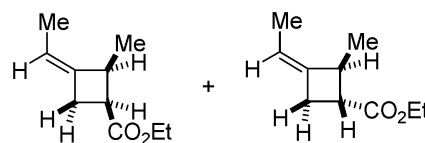

$\mathbf{5 5}(23 \%, 5.8 \%$ ee $) \quad \mathbf{5 6}(16 \%, 8.2 \%$ ee $)$
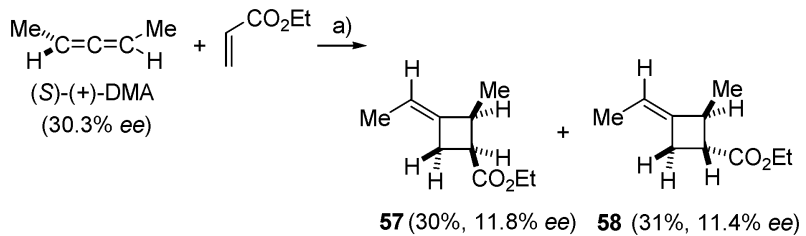

Scheme $20[2+2]$ Cycloaddition of $(S)-(+)$-1,3-dimethylallene with methylacrylate. (a) Reagents and conditions: (a) toluene- $\mathrm{d}_{8}$, hydroquinone, $160{ }^{\circ} \mathrm{C}, 3$ days.
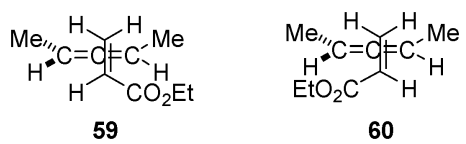

Fig. 1 Approaches of methyl acrylate to 1,3-dimethylallene.

It has been observed that the ee's of the cycloadducts derived from methyl acrylate are significantly higher than those possessed by the cycloadducts with acrylonitrile. Molecular mechanics calculations for the reaction of methyl acrylate with 1,3-dimethylallene have indicated greater preferences for the approaches shown in $\mathbf{5 9}$ and $\mathbf{6 0}$ compared to the approaches in which the ester groups are oriented in an upward manner (Fig. 1).

This may be expected to result in greater diastereoselectivity in the formation of the activated complexes. In addition, the larger size of the methyl ester function compared to the cyano group will result in an increase in the energy barriers for the rotation about the $\mathrm{C} 1-\mathrm{C} 5$ bond and potentially about the $\mathrm{C} 1-\mathrm{C} 2$ bond, thus resulting in less racemization of the intermediates. The formation of the cycloadducts $\mathbf{5 5 - 5 8}$ follows a parallel pathway to that illustrated in Scheme 19 for the formation of the diradical intermediates and cycloadducts with acrylonitrile.

The next step has been the study of the cycloaddition reaction of $N$-phenylmaleimide (NPM) and dimethyl fumarate with an excess of enantioenriched 1,3-dimethylallene ${ }^{29}$ with varying initial enantiomeric excesses of the 1,3-dimethylallene and different concentrations of reactants. These reactions involve the irreversible formation of the diradical intermediates in which a new tetrahedral stereogenic atom is generated. The chirality of these diradical intermediates is "locked in", as a consequence there is no mechanism for the racemization of these intermediates. Thus, it has been observed that the ee of the 1,3-dimethylallene is transferred to the diradical intermediate which appears in the cycloadducts. In addition, the results of molecular modeling calculations have indicated that three separate, minimum energy reaction channels are operative, proceeding through different conformations of the reactants in approaching the activated complexes which result directly in the formation of different minimum-energy conformations of the diradical intermediates. These different conformations of the diradical intermediates undergo ring closure to specific cycloadducts.

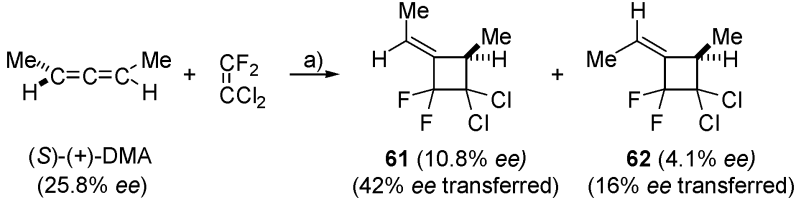

Scheme 21 [2+2] Cycloaddition of $(S)-(+)$-1,3-dimethylallene with 1,1-dichloro-2,2-difluoroethene. (a) Reagents and conditions: diethyl ether, $160{ }^{\circ} \mathrm{C}, 48 \mathrm{~h}$.

Later on, the reaction of enantioenriched $(S)-(+)-1,3-$ dimethylallene with 1,1-dichloro-2,2-difluoroethene was studied. This reaction affords two cycloadducts (Scheme 21). ${ }^{30}$ The major cycloadduct $\mathbf{6 1}$ retained a higher percentage of the ee of the starting 1,3-dimethylallene $(>30 \%)$ than the minor cycloadduct $\mathbf{6 2}$ does $(>10 \%)$. The results of molecular modeling calculations on the conformations of the two reactants in approaching the activated complexes for diradical intermediate formation, and on models for the anti,anti and anti,syn diradical intermediates, have provided a most reasonable interpretation of the results involving one major continuous, low energy reaction pathway via the anti,syn diradical intermediate leading to cycloadduct formation.

Molecular modeling calculations on the conformational preferences for the approach of 1,3-dimethylallene and 1,1dichloro-2,2-difluoroethene have suggested a perpendicular approach of the two reactants and conformation of the diradical intermediates $\mathbf{6 3}$ and $\mathbf{6 4}$ as shown in Fig. 2.

Due to the relatively high ee observed on cycloadduct $\mathbf{6 1}$, it has been concluded that the formation of the anti,syn diradical intermediates must substantially predominate over the formation of the anti,anti diradical intermediate.

A surprising result was obtained in the reaction of enantioenriched $(S)-(+)$-1,3-dimethylallene with 1,1-diphenylethene, which does not lead to detectable formation of [2+2] cycloadducts, ${ }^{30}$ but does result in the racemization of 1,3dimethylallene. These results have been interpreted as it is shown in Scheme 22, which involves the reversible formation of the two diradical intermediates $\mathbf{6 5}$ and $\mathbf{6 6}$. Intermediate $\mathbf{6 5}$ possesses a plane of symmetry, which is formed reversibly resulting in the loss of the optical activity of 1,3-dimethylallene. Intermediate $\mathbf{6 6}$ is chiral, which on cleavage will return to the 1,3-dimethylallene in the same extent of chirality as it inherited from 1,3-dimethylallene. The lack of apparent formation of cycloadducts in the reaction does not mean that they are not formed at all, it only shows that if formed they will be formed in quantities too small to be detected. Cycloadducts 67 and 68

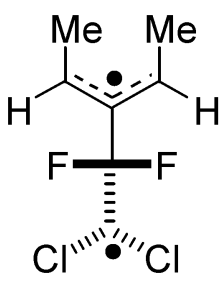

63

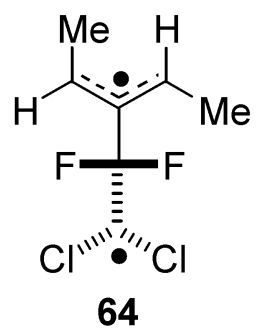

Fig. 2 Diradical intermediates preferred in the [2+2] cycloaddition reaction of 1,1-dimethylallene with 1,1-dichloro-2,2-difluoroethene. 
<smiles>[M]C(C)=C=C=CC</smiles>

(S)-(+)-DMA

$(25.8 \%$ ee)

$\stackrel{+}{\mathrm{H}_{2} \mathrm{C}=\mathrm{CPh}_{2}}$<smiles>C/C=C\C(/C(C)=C\C)C(c1ccccc1)c1ccccc1</smiles><smiles>C#CCCCC</smiles><smiles>C/C=C1/CC(c2ccccc2)(c2ccccc2)C1(C)C</smiles>

67

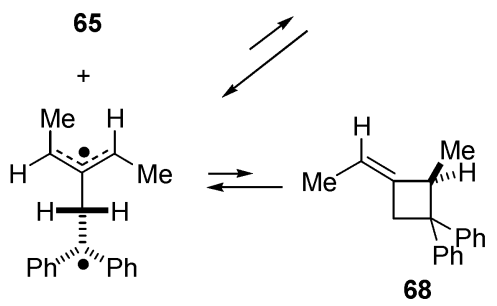

66<smiles>[R]C=CC=C(C)C</smiles>

74

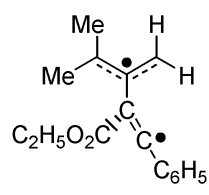

75<smiles>C/C=C(/C(C)=O)[C@H](C(=O)OCC)[C@H](C)OCC</smiles>

76

Fig. 3 Proposed diradicals $\mathbf{7 4 - 7 6}$ for the [2+2] cycloaddition reaction of 1,1-dimethylallene with alkynes.

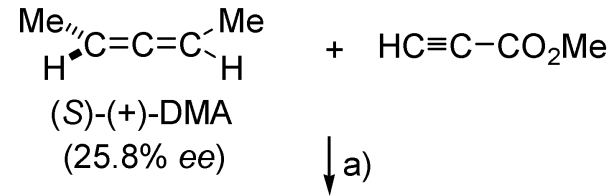

A

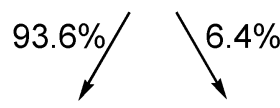

unfavorable equilibria.

On the contrary, the cycloaddition reactions of 1,1-dimethylallene with a number of alkynes have afforded substituted methylenecyclobutenes 69-73, although in some cases isolation by chromatography of cycloadducts was not possible (Scheme 23). ${ }^{31}$

Ring closure of the diradical intermediate $\mathbf{7 4}$ formed occurs exclusively at the less substituted end of the allyl radical, except with ethyl propiolate in which a minor amount of ring closure occurs at the dimethyl-substituted end of the allyl radical. In the cycloaddition reaction with ethyl phenylpropiolate, apparently only the diradical intermediate $\mathbf{7 5}$ is formed which is stabilized by the phenyl group, whereas in $\mathbf{7 6}$ the radical center is stabilized by the ester group (Fig. 3).

Following the series of Pasto's work, the stereochemical features of the $[2+2]$ cycloaddition reaction of 1,3-dimethylallene with methyl propiolate have been reported. ${ }^{32}$ The cycloaddition reaction of enantioenriched 1,3-dimethylallene with methyl propiolate was carried out at $130{ }^{\circ} \mathrm{C}$ affording two cycloadducts 77 and 78 in a ratio of $41.8: 58.2$, along with some remaining unreacted 1,3-dimethylallene (Scheme 24).

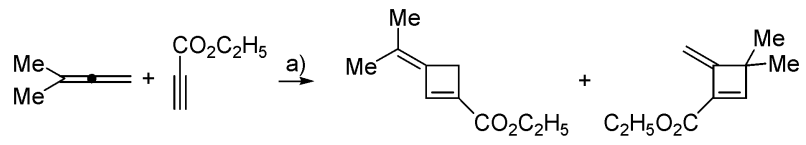
$69(24 \%)$

70<smiles>C=C(C)C(=C)C#CCCCCCC(=O)OCC</smiles><smiles>C#C[C+]=C=C(C)C</smiles>

Scheme 23 [2+2] Cycloaddition of 1,1-dimethylallene with different alkynes. Reagents and conditions: (a) Toluene- $\mathrm{d}_{8}, 160^{\circ} \mathrm{C}, 16 \mathrm{~h}$.<smiles>C/C=C\C(C)CC(C)=O</smiles>

80<smiles>CC=C(C)C=CCC(C)=O</smiles>

81<smiles>[CH]C1C(C(C)=O)=CC1=C(C)C</smiles>

77
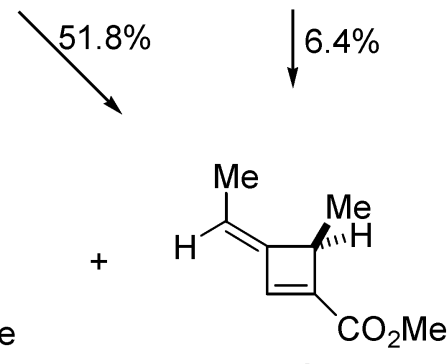

78
(Run 1: $41.7 \% ; 11.7 \%$ ee (Run $1: 58.3 \% ; 10.5 \%$ ee Run 2: $41.9 \% ; 19.8 \%$ ee) $\quad$ Run $2: 58.1 \%, 17.8 \%$ ee)

Scheme $24[2+2]$ Cycloaddition of $(S)-(+)$-1,3-dimethylallene with methylpropiolate. Reagents and conditions: (a) Toluene- $\mathrm{d}_{8}$, hydroquinone, $130{ }^{\circ} \mathrm{C}, 24 \mathrm{~h}^{32}$

A mechanistic scheme for the formation of the cycloadducts 77 and 78 has been proposed. The approach A of methylpropiolate to $(S)$-1,3-dimethylallene is favored over the alternative approach B (Fig. 4).

Collapse of $\mathbf{A}$ to the diradical intermediates $\mathbf{8 0}$ and $\mathbf{8 1}$ will produce the intermediates possessing the absolute configurations shown, with $\mathbf{8 1}$ existing in a perpendicular conformation calculated to be the lowest in energy for the parent system, and $\mathbf{8 0}$ existing in a slightly nonperpendicular conformation (Scheme 24). Racemization of $\mathbf{8 0}$ requires a $180^{\circ}$ rotation about the $\mathrm{C} 1-\mathrm{C} 2$ bond, however, this is not expected to occur. Intermediate 81, however, is achiral and can undergo ring closure to form only racemic $\mathbf{7 8}$. The facts that intermediate $\mathbf{8 1}$ 


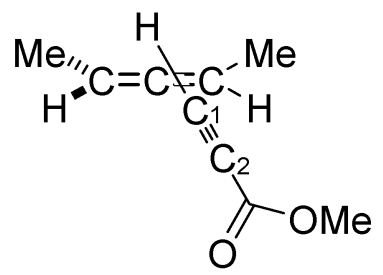

A

Fig. 4 Approaches proposed for the reaction of enantioenriched (S)-1,3-dimethylallene with methylpropiolate.

can give rise to only racemic $\mathbf{7 8}$ and that $\mathbf{7 8}$ possesses an $e e$ comparable to that of $\mathbf{7 7}$ indicate that the majority of $\mathbf{7 8}$ is produced from chiral $\mathbf{8 0}$. If one assumes that both $\mathbf{7 7}$ and $\mathbf{7 8}$ are formed from $\mathbf{8 0}$ with the same extent of transfer of the ee of 80, it is then possible to apportion the relative extent of ring closure of $\mathbf{8 0}$ to the anti and syn ends of the allyl radical intermediates $\mathbf{8 0}$ and $\mathbf{8 1}$. The results have indicated that there is a high preference for the formation of the anti,syn diradical intermediate 80, which prefers to undergo ring closure to the syn-methyl-substituted end of the allyl radical.

In 2000 , Hammond studied the stability of $\alpha$-fluoroallenylphosphonate $\mathbf{8 2}$ under thermal conditions. ${ }^{33}$ Heating compound 82 in a sealed tube furnished dimer 83 exclusively in $73 \%$ yield (Scheme 25). This result contrasts with the mechanistic scenario accounting for a concerted $[2+2]$ process in the allene dimerization. Probably, the bulky phosphorous substituent on the allene moiety allows rotation in the bisallyl diradical intermediate yielding the corresponding tail-to-tail dimer.

Another interesting example showing the dependence of the nature of the substituents attached to the allene moiety in the regiochemistry of the [2+2] cycloadducts has been reported by McGlinchey in 2004. ${ }^{34}$ Thus, during the attempted studies of the Diels-Alder reaction between 9-phenylethynylfluorene 84 and tetracyclone $\mathbf{8 5}$, under thermal conditions, two products resulting from the dimerization of the isomeric allene $\mathbf{8 6}$ were obtained. The major product, 8,16-diphenyl-diindeno[1,2,3$\left.d e: 1^{\prime}, 2^{\prime}, 3^{\prime}-m n\right]$ naphthacene $\mathbf{8 7}$, and a minor component, the dihydronaphthacene $\mathbf{8 8}$ bearing fluorenyl moieties spiro-bonded at the $\mathrm{C} 5$ and $\mathrm{C} 11$ positions were identified (Scheme 26). ${ }^{34}$ Both compounds were not the expected Diels-Alder adducts, but rather are derived from the dimerization of 9-phenylethynylfluorene $\mathbf{8 4}$, presumably via its isomeric allene $\mathbf{8 6}$, with which is in equilibrium. Thus, when the allene $\mathbf{8 6}$ was heated in refluxing heptane two products were formed, a yellow material ( $84 \%$ yield) that was identified as the head-to-tail $[2+2]$ dimer 89, and an unidentified red dimer $(8 \%)$. Therefore, compounds

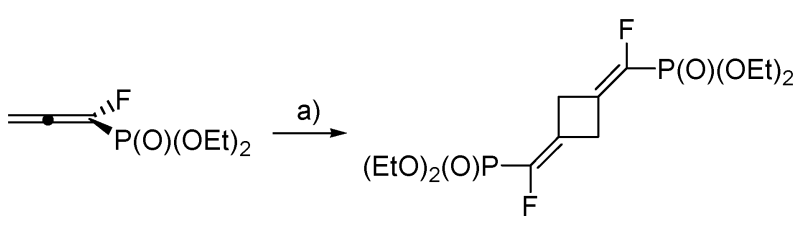
82

$83(73 \%)$

Scheme 25 Dimerization reaction of $\alpha$-fluoroallenylphosphonate 82 . Reagents and conditions: (a) THF, sealed tube, $140{ }^{\circ} \mathrm{C}, 1.5 \mathrm{~h}$.

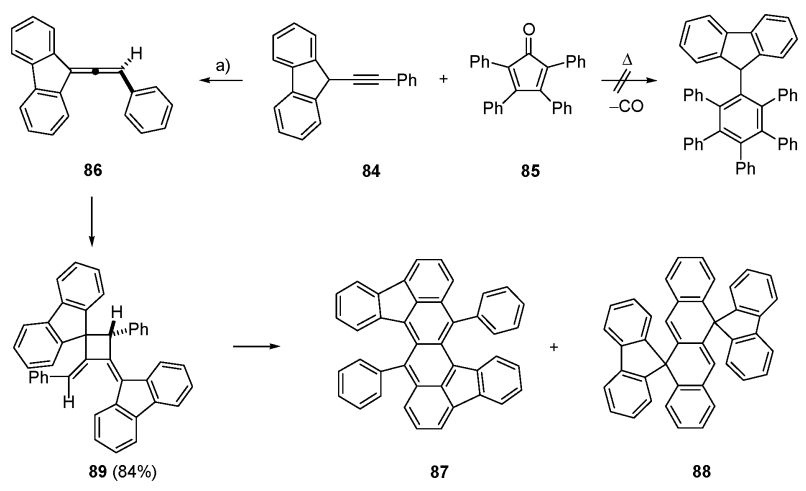

Scheme 26 Thermally induced dimerization reaction of 9-phenylethylnylfluorene 84 via its isomeric allene counterpart 86. Reagents and conditions: (a) Heptane, $\Delta$.

$\mathbf{8 7}$ and $\mathbf{8 8}$ should be formed from dimer $\mathbf{8 9}$ under heating conditions.

More recently, the same authors have described the syntheses and dimerizations of fluorenylidene-derived allenes 90a and 90b to form bis(alkylidene)cyclobutanes 91a and 91b (Scheme 27). ${ }^{35}$ Reaction of 9-ethynyl-9H-fluoren-9-ol 92a with dilute aqueous $\mathrm{HBr}$ at $0{ }^{\circ} \mathrm{C}$ to room temperature for $5 \mathrm{~h}$ gave the corresponding cyclobutane 91a in $85 \%$. On the other hand, reaction of 9-phenylethynylfluorene 92b with triethylamine at $0{ }^{\circ} \mathrm{C}$ to room temperature overnight provided the $[2+2]$ head-to-tail dimer 91b. In both cases, dimers 91a and 91b were obtained without isolation of the precursor allenes 90a and $90 b^{36}$

A stepwise process involving a diallylic diradical intermediate 93 has been proposed. The orthogonal arrangement of the substituents in allenes 90, together with the obvious preference of large groups to minimise steric hindrance, suggests that the initial approaching geometry of two molecules of $\mathbf{9 0}$ is favored by a C2-type trajectory (Scheme 28).

More recently, Swamy has described the $[2+2]$ dimerization of allene 94 during the attempted studies of the Diels-Alder reaction between allene $\mathbf{9 4}$ and antracene under thermal
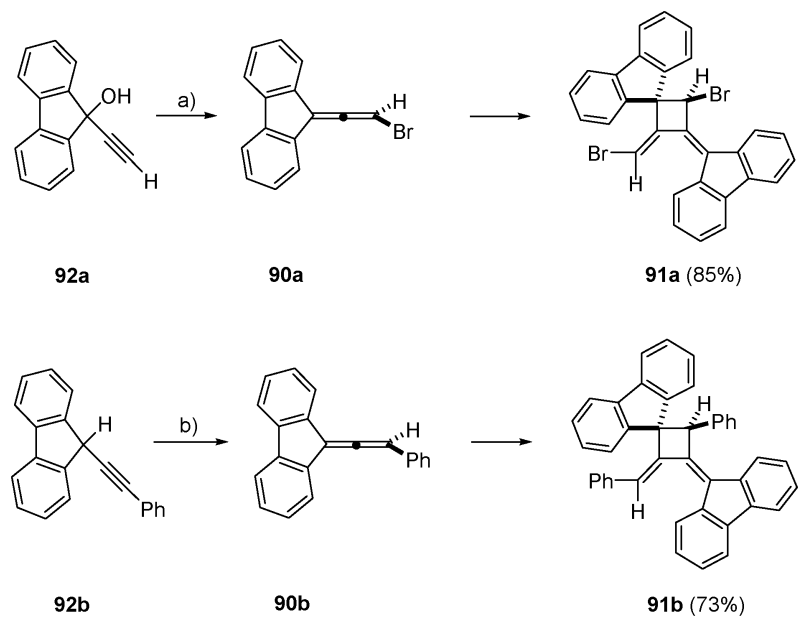

Scheme 27 Dimerization reaction of fluorenylidene-derived allenes 90a and 90b. Reagents and conditions: (a) $\mathrm{AcOH}, \mathrm{HBr}, \mathrm{RT}, 5 \mathrm{~h}$. (b) Pentane, $\mathrm{Et}_{3} \mathrm{~N}, 0{ }^{\circ} \mathrm{C}$ to RT, overnight. 


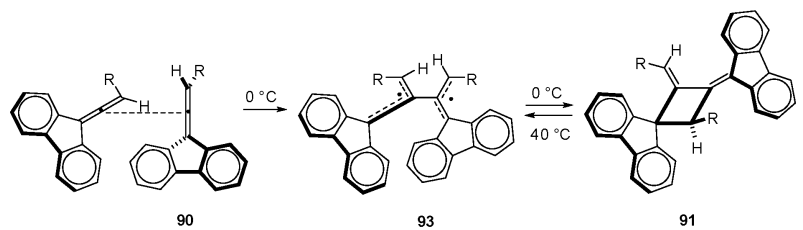

Scheme 28 Proposed mechanism for the formation of dimers 91a and 91b. ${ }^{36}$

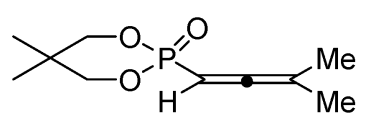

94

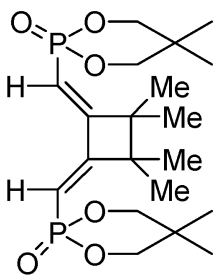

$95(68 \%)$
Scheme 29 Dimerization reaction of phosphorous-derived allene 94 Reagents and conditions: (a) $185^{\circ} \mathrm{C}, 6 \mathrm{~h}$.

conditions. ${ }^{37}$ Head-to-head dimer 95 was obtained in $68 \%$ yield (Scheme 29). A concerted diradical mechanism similar to that shown in Scheme 6 should be involved in the formation of compound 95.

In 2005, Jung and co-workers reported that the cycloaddition of silyloxydienes $96 \mathbf{a}$ and $96 \mathbf{b}$ with allenecarboxylate 97 gave cyclobutanes 98a and 98b in moderate yields (Scheme 30). Hydrolysis of the silyl ethers 98a and $\mathbf{9 8 b}$ in acidic ethanol furnished the corresponding substituted 1-alkenyl-3alkylidenecyclobutanols 99a and 99b in good yields. Finally, treatment of cycloadducts 99a and 99b with a lithium base at low temperature gave the corresponding cyclohexenones 100a and 100b. Apparently, the [2+2] cycloadducts thermally rearrange via a formal [3,3]-sigmatropic shift, providing the corresponding endo adducts 100a and 100b diastereoselectively. ${ }^{38}$ The versatility of the above methodology

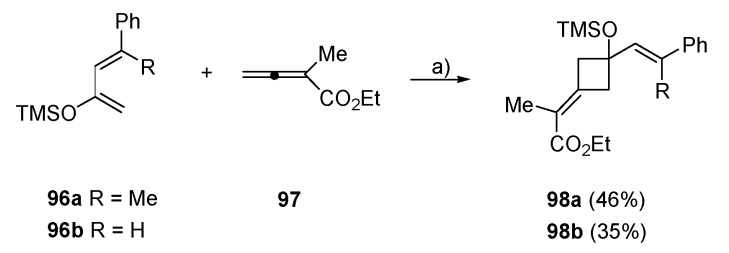

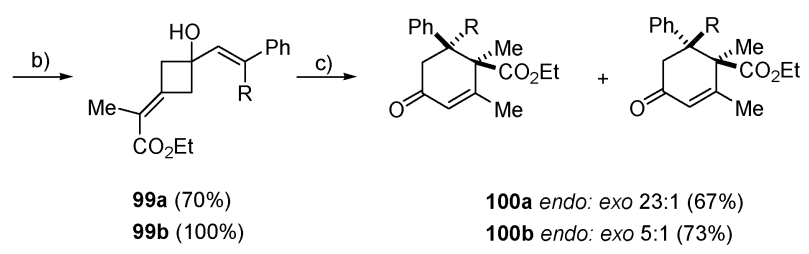

Scheme 30 [2+2] Cycloaddition reaction of 2-silyloxydienes 96a and 96b with allenecarboxylate 97. Reagents and conditions: (a) Toluene, 80 or $130{ }^{\circ} \mathrm{C}$, pressure tube, $24-48$ h. (b) $p$-TsOH, EtOH, THF, RT, 2h. (c) LiHMDS, -78 to $0{ }^{\circ} \mathrm{C}, 1.5 \mathrm{~h}$.

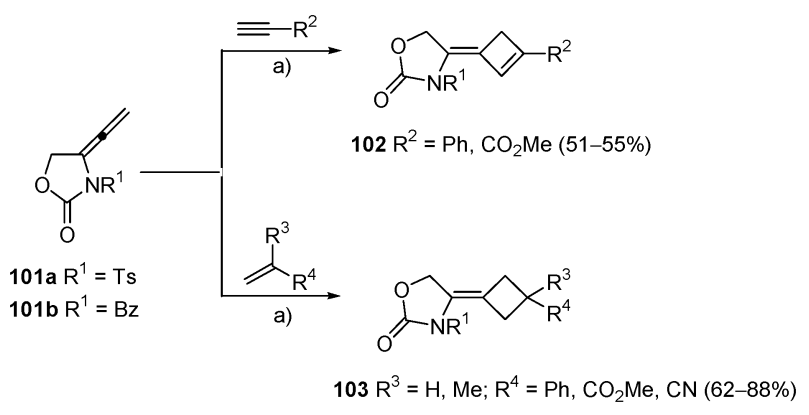

Scheme 31 Intermolecular [2+2] cycloaddition of 4-vinylideneoxazolidin-2-ones 101 with several terminal alkynes and alkenes. Reagents and conditions: (a) Alkyne or alkene as solvent, $80-100{ }^{\circ} \mathrm{C}, \mathrm{N}_{2}, 4-48 \mathrm{~h}$.

has been applied to the enantioselective synthesis of (-)-dysidiolide $^{39}( \pm)$-hedychenone ${ }^{40}$ and $( \pm)$-hedychilactone $\mathrm{B}^{41}$ via stepwise allenoate diene cycloaddition.

In 2003, Tamaru developed the thermal intermolecular $[2+2]$ cycloaddition of vinylideneoxazolidin-2-ones 101a-c with terminal alkynes, alkenes, and 1,3-dienes, regardless of their electronic nature, showing a exclusive preference over the $[4+2]$ cycloaddition. Thus, the corresponding 3-substituted (Z)-methylene-cyclobutenes 102, 3-substituted methylenecyclobutanes 103, and 3-vinylmethylene-cyclobutanes 104, have been obtained in good to excellent yields. ${ }^{42}$ Firstly, the $[2+2]$ cycloaddition of vinylideneoxazolidin-2-ones 101 with alkynes was investigated. Treatment of compounds 101 with an excess of phenylacetylene or ethyl propiolate at $100{ }^{\circ} \mathrm{C}$ afforded the corresponding methylene-cyclobutanes (Z)-102, as single isomers in good yields. Surprisingly, of the two conceivable stereoisomers, only $\mathrm{Z}$ isomers were isolated (Scheme 31). Next, the authors examined the reaction of vinylideneoxazolidin-2-ones $\mathbf{1 0 1}$ with alkenes of different structural and electronic substituents. In general, alkenes bearing conjugated $\pi$-systems and electron-withdrawing groups showed parallel results, obtaining the corresponding $[2+2]$ cycloadducts $\mathbf{1 0 3}$ in good yields. However, alkenes bearing electron-donating substituents displayed a completely different reactivity yielding tetrahydropyridines via a formal $[4+2]$ cycloaddition reaction.

Analogously, vinylideneoxazolidin-2-ones 101a and 101c displayed no dienophilic reactivity toward dienes, but instead, selectively underwent [2+2] cycloaddition to provide 3-vinyl1-methylene-cyclobutanes $\mathbf{1 0 4 a - j}$ in good to excellent yields (Scheme 32).

The regioselectivity observed for the [2+2] cycloaddition of allenes 101a and 101c with dienes, depends on the nature of the substituents attached to the diene moiety. Thus, 1-substituted dienes react at the distal non-substituted 3,4-double bonds (104c, 104h and 104i), while 2-substituted dienes react selectively at the 1,2-double bonds, irrespectively of the electronic nature of the substituents $(\mathbf{1 0 4 b}, \mathbf{1 0 4 f}$ and $\mathbf{1 0 4 g}$ ), and unsymmetrically 2,3-substituted dienes react selectively at the double bonds with higher electron density (104j).

The mechanistic details associated with the thermal $[2+2]$ cycloaddition of 4-vinylidenoxazolidin-2-ones 101a-c have pointed to a concerted mechanism, involving a six-electron Hückel $\left[\left(\pi_{2 \mathrm{~s}}+\pi_{2 \mathrm{~s}}\right)_{\text {allene }}+\pi_{2 \mathrm{~s}}\right]$ transition state, based on the 
<smiles>C=C=C1COC(=O)[NH2+]1</smiles>

$101 a$
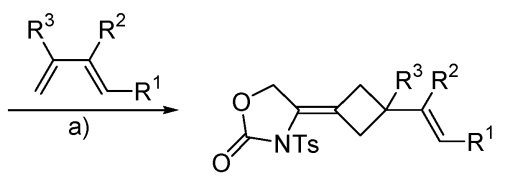

104a $R^{1}=H, R^{2}=R^{3}=\operatorname{Me}(75 \%)$ 104b $R^{1}=R^{2}=H, R^{3}=\mathrm{CH}_{2}$ TMS $(80 \%)$ 104c $R^{1}=P h, R^{2}=R^{3}=H(30 \%)$ 104d $R^{1}=P h, R^{2}=H, R^{3}=$ OTIPS $(73 \%)$
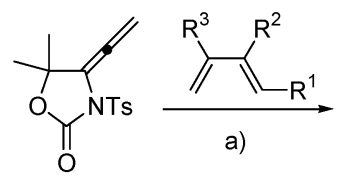

$101 c$

$$
\begin{aligned}
& \text { 104e } R^{1}=H, R^{2}=R^{3}=M e(68 \%) \\
& \text { 104f } R^{1}=R^{2}=H, R^{3}=P h(70 \%) \\
& 104 g R^{1}=R^{2}=H, R^{3}=C_{2} \text { TMS }(90 \%) \\
& \text { 104h } R^{1}=P h, R^{2}=R^{3}=H(65 \%) \\
& \text { 104i } R^{1}=\text { OTIPS, } R^{2}=R^{3}=H(86 \%) \\
& \text { 104j } R^{1}=H, R^{2}=M e, R^{3}=\text { OTIPS }(80 \%)
\end{aligned}
$$

Scheme 32 Intermolecular [2+2] cycloaddition between 4-vinylideneoxazolidin-2-ones 101a and 101c with 1,3-dienes. Reagents and conditions: (a) Dioxane, $80-100{ }^{\circ} \mathrm{C}, \mathrm{N}_{2}, 9-24 \mathrm{~h}$.

frontier orbital theory (Fig. 5). The unique reactivity associated with 101 might be attributed to a strong $\sigma^{*}\left(\mathrm{~N}-\mathrm{SO}_{2}\right)$ $\pi^{*}\left(\mathrm{C}_{\alpha}=\mathrm{C}_{\beta}\right)$ interaction of 101a and 101c [or $\sigma^{*}(\mathrm{~N}-\mathrm{CO})-$ $\pi^{*}\left(\mathrm{C}_{\alpha}=\mathrm{C}_{\beta}\right)$ interaction for 101b], which produces (1) the lowering of the $\pi^{*}\left(C_{\alpha}=C_{\beta}\right)$ energy level (and hence, lowering of the activation energy for the [2+2] addition) and (2) the rehybridization of the $\pi^{*}\left(\mathrm{C}_{\alpha}=\mathrm{C}_{\beta}\right)$ orbitals ( $\mathrm{sp}^{3}$-like, and hence, better overlap between the p orbitals of alkene $\mathrm{Cl}$ and allene $\mathrm{C}_{\beta}$ in the opposite face to N-Ts). As it is shown in
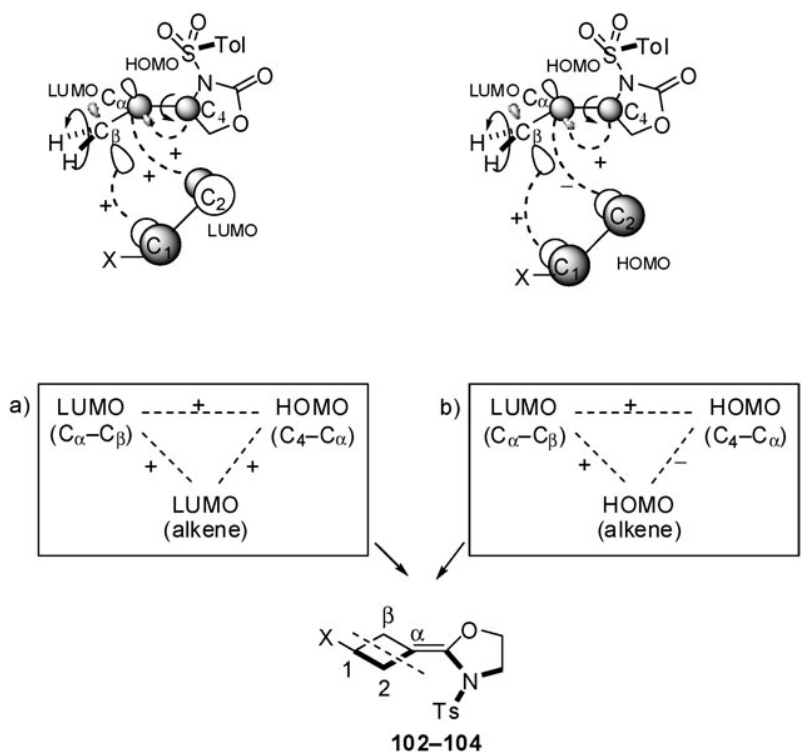

Fig. 5 Explanation for the thermal, concerted [2+2] cycloaddition on "the orbital interaction in three-systems": (a) reactions for electron-deficient alkenes and alkynes, (b) reactions for electron-rich and conjugated alkenes and alkynes. ${ }^{42}$
Fig. 5a for electron-deficient alkynes and alkenes and in Fig. 5b for electron-rich and conjugated alkynes and alkenes, the three-systems interaction, with a coaxial overlap of the $\mathrm{p}$ orbitals of $\mathrm{C}_{\alpha}$ and $\mathrm{C} 2$ and a perpendicular overlap of the $\mathrm{p}$ orbitals of $\mathrm{C}_{\beta}$ and $\mathrm{C} 1$, necessitates a $90^{\circ}$ counter-clockwise rotation of the oxazolidinone ring (i.e., a rotation of the $N$-Ts group toward C2) to furnish compounds (Z)-102-104.

In 2002 Nair and coworkers studied the [2+2] cycloaddition of allenes with [60]fullerene for the preparation of novel cyclobutane annulated fullerene derivatives. Thus, treatment of allenamides $\mathbf{1 0 5}$ with [60]fullerene in refluxing toluene has afforded the corresponding cyclobutane annulated fullerene derivatives 106 in moderate yields (Scheme 33). ${ }^{43}$ The mechanism of the reaction has been rationalized as outlined in Scheme 33. The electron deficient fullerene cage can stabilize the negative charge, thereby facilitating the intramolecular attack to the iminium moiety. A possible charge transfer complex formation between the allenamide $\mathbf{1 0 5}$ and [60]fullerene, which will bring the $\mathrm{C}^{\prime}-\mathrm{C} 2^{\prime}$ double bond of the allene in close proximity to the cage, would facilitate the $[2+2]$ cycloaddition.

The $[2+2]$ cycloaddition reaction has also been promoted under high pressure conditions. In particular, in 2003 Scheeren group studied the reaction of enol ethers $\mathbf{1 0 7}$ with the 1,2propadienyl sulfone $\mathbf{1 0 8}$ at $15 \mathrm{kbar}$, providing regioselectively the corresponding (3-alkoxycyclobutylidene)methyl 4-methylphenyl sulfones 109 in good yields (62-75\%) (Scheme 34). ${ }^{44}$ Interestingly, substitution on the enol ether moiety does not affect the regioselectivity of the reaction, which is determined by the electron-richness of the reacting alkene.

Very recently, in 2009 Ogasawara ${ }^{45}$ described the stereoselective intermolecular [2+2] cycloaddition of allenes $\mathbf{1 1 0}$ with ketenes 111, affording a mixture of several isomers
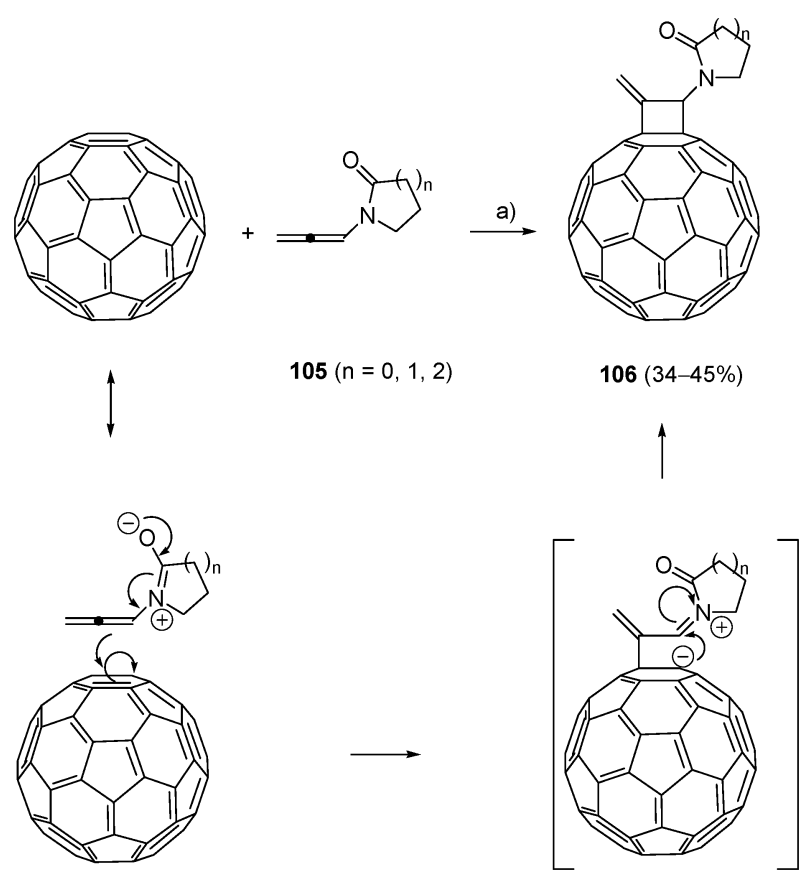

Scheme 33 Reaction and proposed mechanism for the $[2+2]$ cycloaddition reaction between allenamides 105 and [60]fullerene. Reagents and conditions: (a) Toluene, $110{ }^{\circ} \mathrm{C}, 12 \mathrm{~h} .{ }^{43}$ 


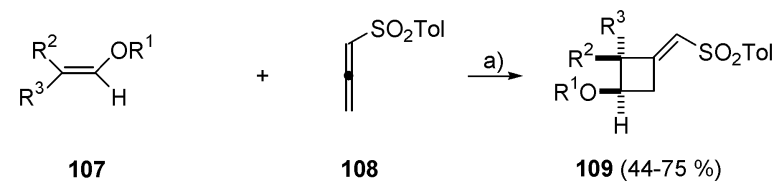

$\mathrm{R}^{1}=\mathrm{Me}, \mathrm{Et}, \mathrm{iPr}, \mathrm{tBu}, \mathrm{PhCHMe}$

$R^{2}=H, M e ; R^{3}=H, M e$

Scheme 34 High pressure-promoted [2+2] cycloaddition of 4-methylphenyl-1,2-propadienyl sulfone $\mathbf{1 0 8}$ with enol ethers $\mathbf{1 0 7}$. Reagents and conditions: (a) HP (15 kbar), RT or $50{ }^{\circ} \mathrm{C}, 16 \mathrm{~h}$.

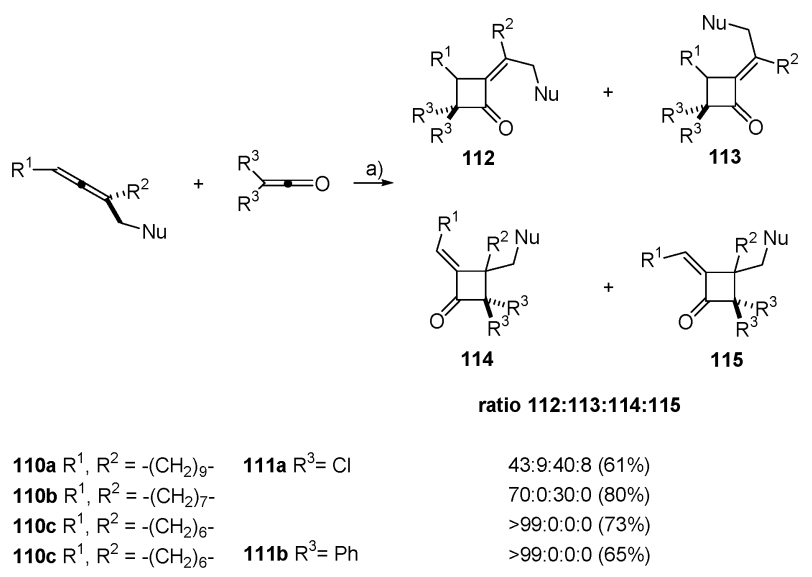

$\mathrm{Nu}=\mathrm{CMe}\left(\mathrm{CO}_{2} \mathrm{Me}\right)_{2}$

Scheme 35 Intermolecular [2+2] cycloaddition of allenes with ketenes. Reagents and conditions: (a) Ether/DME, $23{ }^{\circ} \mathrm{C}, 8 \mathrm{~h}$.

depending on the nature of the endocyclic structure of the starting allene (Scheme 35). Thus, the reaction of the cyclic allene 110a with dichloroketene 111a produced a mixture of four isomers. However, the smaller-membered cyclic allenes exhibited different stereoselectivity in the [2+2] cycloaddition. As an example, reaction of $\mathbf{1 1 0 b}$ with ketene 111a afforded only two isomers. More remarkably, the reaction between nine-membered 110c with 111a and 111b gave adducts 112 as single isomers in 73 and $65 \%$ yield, respectively. The results obtained have indicated that the cyclic structures in the smaller endocyclic allenes direct a reacting ketene from the unencumbered face as expected (see Fig. 6).

In addition, the authors have studied the axial-to-central chirality transfer by the asymmetric synthesis of the scalemic endocyclic allene 116. Reaction of allene $\mathbf{1 1 6}$ with ketene 111a afforded the [2+2] cycloaddition product enantiomerically enriched 117 in $64 \%$ ee and $60 \%$ yield. ${ }^{45}$ The chirality transfer of the reaction was estimated to be $>98 \%$ (Scheme 36).

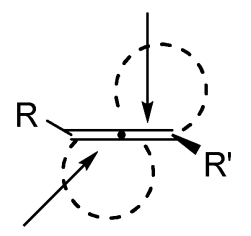

Fig. 6 Steric properties of endocyclic allenes.

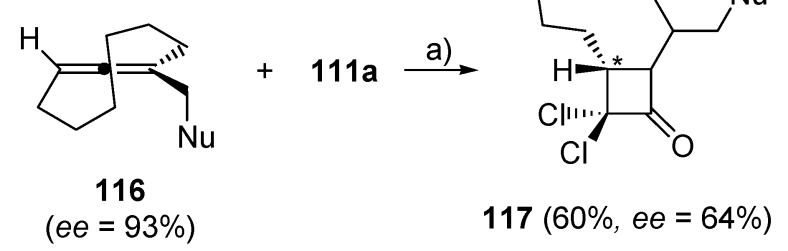

$\mathrm{Nu}=\mathrm{CMe}\left(\mathrm{CO}_{2} \mathrm{Me}\right)_{2}$

Scheme $36[2+2]$ Intermolecular cycloaddition of enriched allene 116 with ketene 111a. Reagents and conditions: (a) Ether/DME, $23{ }^{\circ} \mathrm{C}, 8 \mathrm{~h}$.

\subsection{Intramolecular $[2+2]$ cycloadditions under thermal conditions}

Considering the intramolecular [2+2] cycloaddition of allenenes and allenynes 118, two regioisomers can be formed, the proximal cycloadduct 119 by reaction with the internal $2 \pi$ component, and the distal cycloadduct $\mathbf{1 2 0}$ by reaction with the external $2 \pi$ component of the allene moiety (Scheme 37 ).

The intramolecular thermal $[2+2]$ cycloaddition of allenenes and allenynes has offered an interesting opportunity to prepare selectively polycyclic compounds. In general, the regioselectivity of the cycloaddition depends on the steric nature of the substituents attached to the allene or alkene/alkyne moieties, providing the corresponding proximal or distal adducts.

Pioneering works by Padwa's group have shown the dependence of the substituents of the allene moiety $(\alpha$ - or $\gamma$-tethered allenyl sulfones) into the thermal intramolecular $[2+2]$ cycloaddition. ${ }^{46}$ Thus, the thermal $[2+2]$ cycloaddition of several $\alpha$-tethered allenyl sulfones $\mathbf{1 2 1}$ took place across the non-activated $\pi$-system of the allene, affording the corresponding distal cycloadducts $\mathbf{1 2 2}$ in excellent yields with complete stereospecificity (Scheme 38).

Formation of cycloadducts $\mathbf{1 2 2}$ can be explained as depicted in Scheme 39. The initial rate-determining carbon-carbon bond formation occurs between the central allene carbon and the proximal alkene carbon atom. Although the regioselectivity on the alkene system is due to less clear-cut

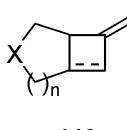

119

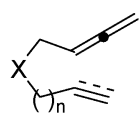

118<smiles></smiles>

120
Proximal adduct

Scheme 37 Possible regioisomers observed in the intramolecular $[2+2]$ cycloaddition of allenenes and allenynes.<smiles>[R]C([R])=CCC(CC(=CC)C(=O)Oc1ccccc1)(S(=O)(=O)O)S(=O)(=O)Oc1ccccc1</smiles>

$121 \mathrm{R}^{1}=\mathrm{H}, \mathrm{Me}, \mathrm{R}^{2}=\mathrm{H}, \mathrm{Me}$

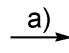

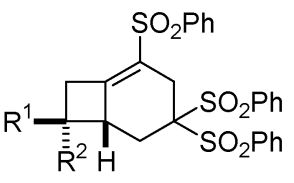

$122(80-100 \%)$

Scheme 38 [2+2] Cycloaddition of $\alpha$-tethered allenyl sulfones 121 . Reagents and conditions: (a) Benzene, $80{ }^{\circ} \mathrm{C}, 45 \mathrm{~min}-22 \mathrm{~h}$. 


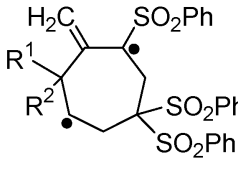

124

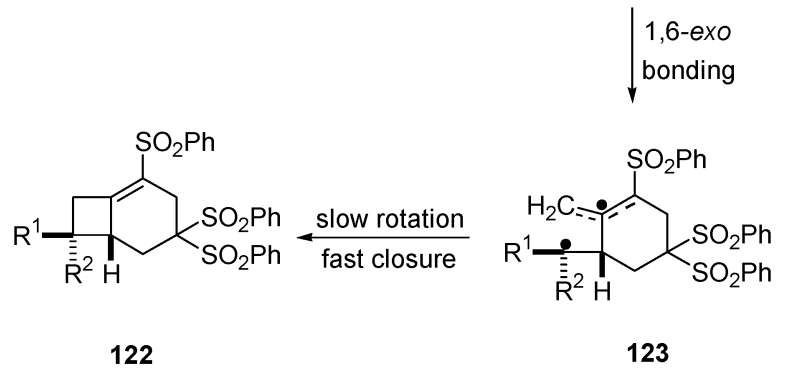

Scheme 39 Stereospecificity of the [2+2] cycloaddition of allenyl sulfones $121 .^{46}$

parameters. Evidently, it is easier for stepwise bonding to occur in a 1,6-exo manner (leading to diradical 123) than in a 1,7-endo fashion (through diradical 124).

An interesting change in the regioselectivity of the $[2+2]$ cycloaddition has been observed with allenes 125. In fact, heating allenes $\mathbf{1 2 5}$ in xylene produced the proximal cycloadducts 126, which correspond to a formal [2+2] cycloaddition across the more activated double bond (Scheme 40). Thus, substitution at the 7-position effectively caused a crossover in the allene regioselectivity. These reactions proceeded through a similar mechanism, in which the central allene carbon is involved in the initial carbon-carbon bond formation with the distal alkene carbon, to give an intermediate diradical 127. The resulting methylenecycloheptyl diradical 127 undergoes subsequent closure to form the bicyclo[3.2.0]heptane ring 126.

Alkynes can isomerize to the corresponding allenes under selected conditions. This process is favored if the migrating $\pi$-bond in conjugation with an alkene, alkyne or aromatic ring and usually this reaction is performed under basic conditions employing $\mathrm{KOH}, \mathrm{Na}_{2} \mathrm{CO}_{3}$ or $\mathrm{KOtBu}$. This isomerization has also proven to be very useful in the $[2+2]$ cycloaddition of allenes. Thus, the $[2+2]$ cycloaddition has been studied in $\gamma$-tethered-allenes 128. Treatment of propargyl sulfones 129 with triethylamine under thermal conditions allowed for equilibrium concentrations of allenes $\mathbf{1 2 8}$ to be formed, which then underwent rapid intramolecular cycloaddition to give the methylenecyclobutanes 130 (Scheme 41). ${ }^{47}$ A diradical intermediate $\mathbf{1 3 1}$ arising from initial attack of the terminal olefinic carbon onto the central allene carbon atom should be involved in the formation of tricycles 130. It is assumed that the

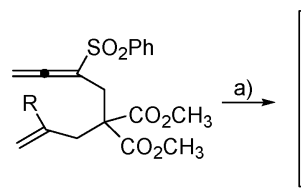

$125 \mathrm{R}=\mathrm{Me}, \mathrm{Br}$

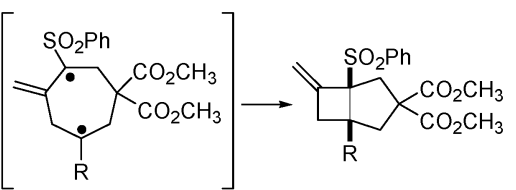

127

$126(64-77 \%)$
Scheme 40 Change in the regioselectivity of the [2+2] cycloaddition in $\alpha$-tethered-allene systems 125. Reagents and conditions: (a) Xylenes, reflux, 6-12 h. ${ }^{46}$<smiles>[R]C([R])=C([R])Cc1ccccc1C#CC[SH](C)(=O)O</smiles>

$\mathrm{R}^{1}=\mathrm{H} ; \mathrm{R}^{2}=\mathrm{H}, \mathrm{Me} ; \mathrm{R}^{3}=\mathrm{H}, \mathrm{Me}$

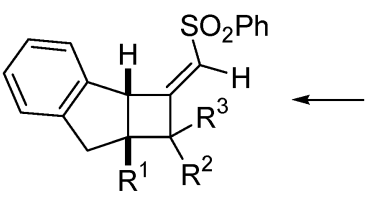

$130(82-89 \%)$<smiles>[R]C([R])=C([R])Cc1ccccc1C=CC(=O)OS(=O)(=O)c1ccccc1</smiles>

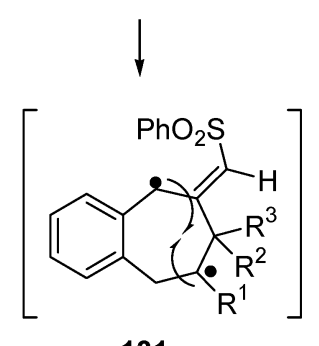

131
Scheme 41 Intramolecular [2+2] cycloaddition in $\gamma$-tethered-allene systems 128. Reagents and conditions: (a) Benzene, $\mathrm{Et}_{3} \mathrm{~N}$ (cat.), reflux, 5-24 h. ${ }^{47}$

resulting diradical intermediate $\mathbf{1 3 1}$ would rapidly close (before bond rotation can occur) to the more stable cis-fused 4,5-ring systems $\mathbf{1 3 0 .}$

The regiochemical outcome observed in allenes $\mathbf{1 2 5}$ is in agreement with the results reported by Strenstrøm in 2003. Thus, the intramolecular [2+2] cycloaddition on phenylsulfonyl allene $\mathbf{1 3 2}$ has been studied in order to prepare $( \pm)-\Delta^{6}$-protoilludene. However, thermal treatment of allene 132 provided the tricyclic structure 133, with the observed regiochemistry of the [2+2] cycloaddition being opposite to the desired one (Scheme 42). ${ }^{48}$ A seven-membered diradical intermediate analogue to $\mathbf{1 2 7}$ should explain the formation of compound $\mathbf{1 3 3}$.

In our research group, we have used the thermal $[2+2]$ cycloaddition of 2-azetidinone-tethered enallenols for the synthesis<smiles>C=C1C(S(=O)(=O)O)=C(C)[C@@H]2C[C@@H]1CC(C)(C)C2</smiles>

132<smiles>[3H]C([3H])=C</smiles><smiles>CC1(C)C[C@H]2CC(S(=O)(=O)Oc3ccccc3)=C3CC[C@@H]3[C@H]2C1</smiles>

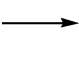

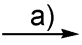<smiles>C=C1C[C@]2(C)[C@H]3CC(C)(C)C[C@H]3C[C@]12S(=O)(=O)c1ccccc1</smiles>

$133(28 \%)$<smiles>CC1=C2CC[C@H]2[C@@H]2CC(C)(C)C[C@H]2C1</smiles>

\section{$( \pm)-\Delta^{6}$-protoilludene}

Scheme 42 Intramolecular [2+2] cycloaddition of phenylsulfonyl allene 132. Reagents and conditions: (a) Xylenes, $\Delta, 80 \mathrm{~h}$. 
<smiles>[R]C(=C)CN1C(=O)[C@H](OC)[C@H](O)[C@H]1[C@H](O)C([R1])=C</smiles>

134 $\mathrm{R}^{1}=\mathrm{H}, \mathrm{Me}, \mathrm{Ph} ; \mathrm{R}^{2}=\mathrm{H}, \mathrm{Me}$

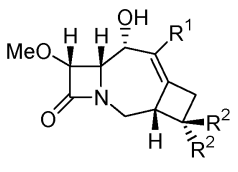

$136(40-58 \%)$
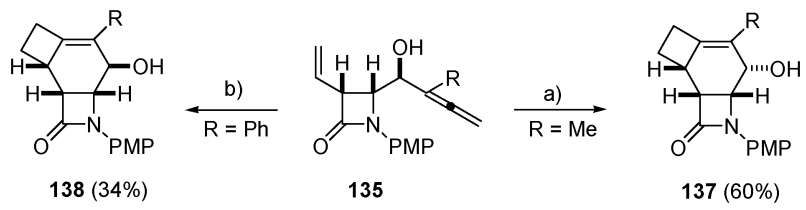

Scheme 43 Synthesis of tricyclic $\beta$-lactams 136-138 by formal [2+2] cycloaddition reaction of 2-azetidinone-tethered enallenols 134 and 135. Reagents and conditions: (a) Toluene, $220{ }^{\circ} \mathrm{C}$, sealed tube, for $\mathrm{R}^{1}=\mathrm{H}: 10 \mathrm{~h}$; for $\mathrm{R}^{1}=\mathrm{Me}, \mathrm{Ph}: 1.5-8 \mathrm{~h}$.

of racemic and enantiopure strained tricyclic $\beta$-lactams containing a cyclobutane ring. ${ }^{49}$ It was observed that the regioselectivity of this cycloaddition reaction is determined by the presence or absence of an alkyl substituent at the internal alkene carbon atom. Thus, treatment of allenenes 134 and 135 in toluene at $220{ }^{\circ} \mathrm{C}$ in a sealed tube afforded tricyclic $\beta$-lactams 136-138, in reasonable yields and complete regio- and diastereoselectivity, (Scheme 43). The tricyclic ring structures 136-138 arise from the formal [2+2] cycloaddition of the alkene with the distal bond of the allene moiety. Interestingly, the regioselectivity of the process is not affected by the substitution at the allene group.

On the other hand, it was observed that the presence of an internal substituent at the alkene moiety switched the regioselectivity. Thus, exposure of allenols 139-141 to the above thermal conditions afforded methylenecyclobutane 2-azetidinones 142-146 as the only products in reasonable yields (Scheme 44).
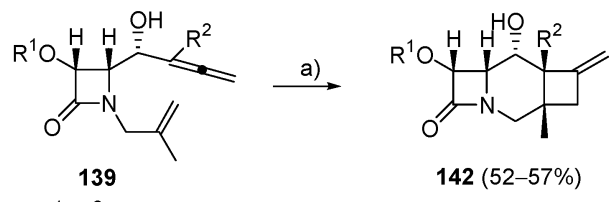

$\mathrm{R}^{1}, \mathrm{R}^{2}=\mathrm{Me}, \mathrm{Ph}$
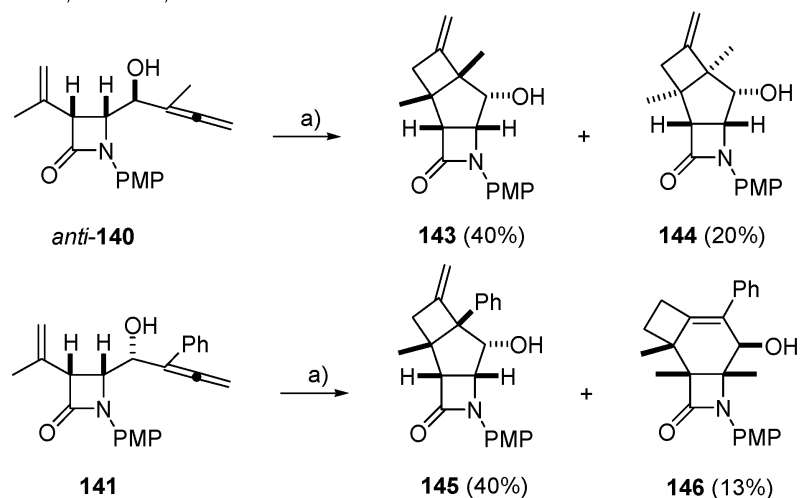

$144(20 \%)$

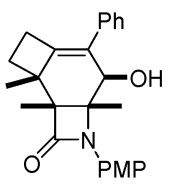

$146(13 \%)$

Scheme 44 Change in the regioselectivity of the intramolecular [2+2] cycloaddition reaction of 2-azetidinone-tethered enallenols 139-141. Reagents and conditions: (a) Toluene, $220^{\circ} \mathrm{C}$, sealed tube, 5-7 h.
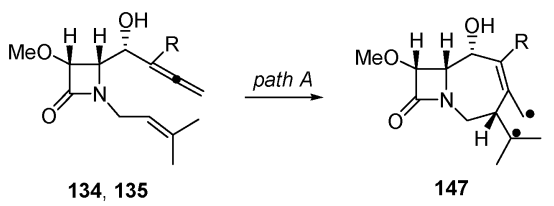

147

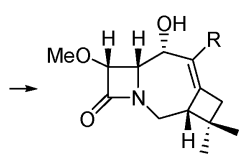

136-138
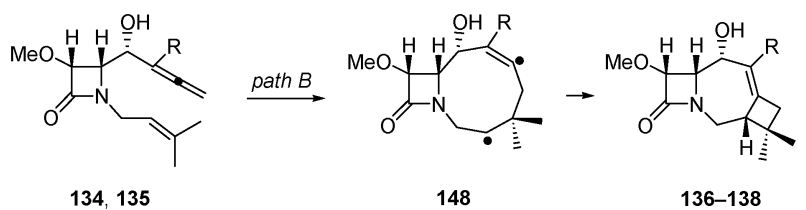

Scheme 45 Radical mechanistic proposals for the formation of strained tricycles 136-138. ${ }^{49}$

Formation of fused strained tricycles 136-138 has been rationalized by a radical mechanism involving an exocyclic diradical intermediate 147 through initial carbon-carbon bond formation between the central allene and proximal alkene carbon atoms (path $A$, Scheme 45). An alternative mechanism may also be proposed (path $B$, Scheme 45 ). This pathway would involve and endocyclic diradical intermediate 148, arising from the initial attack of the terminal olefinic carbon onto the distal allene carbon. Both pathways would include a rapid ring closure of the diradical intermediates before bond rotation.

Analogously, formation of tricycles 142-145 has been rationalized by a mechanism which includes an exocyclic diradical intermediate 149 through initial carbon-carbon bond formation involving the proximal allene and internal alkene carbon atoms (path $C$, Scheme 46). An alternative pathway leading to compounds 142-145 has also been proposed in path $D$. This proposal would involve an endocyclic diradical intermediate 150, arising from the initial attack of the terminal olefinic carbon onto the central allene carbon. The final ring-closing step of the diradical intermediates would account for the cyclobutane formation.

It seems that the regioselectivity in this type of $[2+2]$ cycloaddition reaction is determined by the presence or absence of an alkyl substituent at the internal alkene carbon atom, as the allenenes $\mathbf{1 3 4}$ and $\mathbf{1 3 5}$ that are lacking a methyl group exclusively produced addition at the $\beta, \gamma$-double bond, while the allenenes 139 and $\mathbf{1 4 1}$, which bear a methyl group at the internal olefinic carbon underwent a formal $[2+2]$ cycloaddition reaction at the $\alpha, \beta$-double bond. Path $A$ (Scheme 45) looks valid for the formation of products 136-138. For this case, the Me group stabilizes the exocyclic
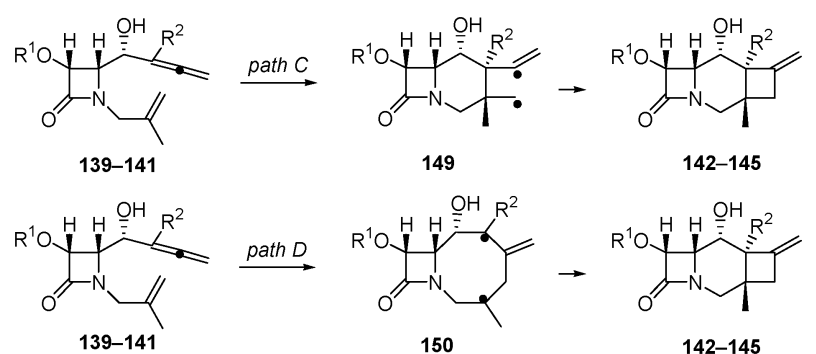

Scheme 46 Radical mechanistic proposals for the formation of strained tricycles $\mathbf{1 4 2 - 1 4 5 .}{ }^{49}$ 
<smiles>[R7]C=CCN(C)C(C=C)C(C)C</smiles>

$$
\begin{aligned}
& 151 \mathrm{a} \mathrm{R}^{1}=\mathrm{Ph} \\
& 151 \mathrm{~b} \mathrm{R}^{1}=4-\mathrm{MeO}^{-} \mathrm{C}_{6} \mathrm{H}_{4} \\
& 151 \mathrm{c} \mathrm{R}^{1}=4-\mathrm{NO}_{2}-\mathrm{C}_{6} \mathrm{H}_{4} \\
& 151 \mathrm{~d} \mathrm{R}^{1}=\mathrm{CO}_{2} \mathrm{Me}
\end{aligned}
$$<smiles>[X]CC/C=C\C(=C)C/C=C\c1ccccc1</smiles>

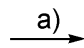

$151 \mathrm{e} X=0$ $151 \mathrm{X}=\mathrm{C}\left(\mathrm{CO}_{2} \mathrm{Me}\right)_{2}$

Scheme 47 Thermal intramolecular [2+2] cycloaddition reaction of allenenes 151a-f. Reagents and conditions: (a) DMF, $150{ }^{\circ} \mathrm{C}, 1-30 \mathrm{~h}$.

diradical, and the presence of the double bond promotes the allylic radical 147 over the alternative endocyclic vinylic radical 148 in path $B$. However, it could be presumed that for the formation of compounds 142-145, path $D$ (Scheme 45) is more reasonable. The simultaneous stabilization of the endocyclic diradical $\mathbf{1 5 0}$ by the presence of a methyl substituent and allylic stabilization makes this radical favoured over the exocyclic diradical 149.

In a more recent work, Ohno and Tanaka have presented a stereoselective route to [4.2.0] octane derivatives using thermal $[2+2]$ cycloaddition of unactivated allenenes $\mathbf{1 5 1} \mathbf{a}-\mathbf{j} .{ }^{50}$ The reactions were carried out using dimethylformamide as the solvent at $150{ }^{\circ} \mathrm{C}$, leading to 3-azabiclo[4.2.0]oct-5-ene derivatives 152a-j in good to excellent yields, with complete

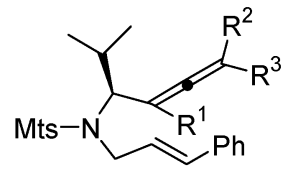

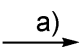

(3:1) mixture of diastereomers

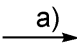

$$
\begin{aligned}
& \text { 151i } R^{1}=H, R^{2}=M e \\
& \text { 151j } R^{1}=R^{2}=M e
\end{aligned}
$$<smiles>[R]C1=C2C(C(C)C)N(S(C)(=O)=O)C2[C@H](c2ccccc2)C1([R])[R]</smiles>

$152 g(90 \%)$

$152 \mathrm{~h}(46 \%)$

$$
\begin{aligned}
& 151 g R^{1}=M e, R^{2}=R^{3}=H \\
& 151 h R^{1}=H, R^{2}=R^{3}=M e
\end{aligned}
$$<smiles>[R]C([R])=CC(C)(C)N(C)S(C)(C)C=Cc1ccccc1</smiles><smiles>[R]C/C=C\C([R])N(P)CCC</smiles>

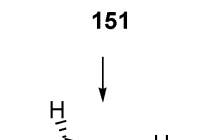<smiles>[R]/C=C\C=C(C)C([R])N(P)/C=C\C</smiles><smiles>[R]C([2H])C1CC(P)CC(P)C1</smiles><smiles>[V]=[Tl]</smiles>

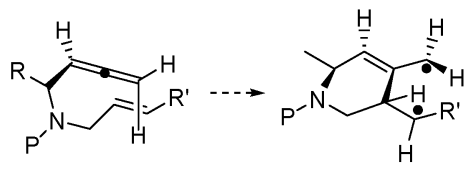

154<smiles>[R]C1CC2=CC([R7])N(P)C[C@@H]21</smiles>

156 not isolated single isomer

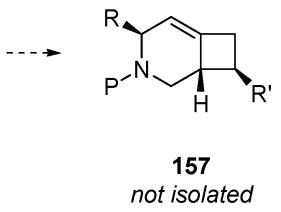

Scheme 49 Mechanistic proposal accounting for the stereoselectivity of the thermal intramolecular [2+2] cycloaddition of allenenes $150 .{ }^{50}$

regioselectivity (Scheme 47 and 48). The [2+2] cycloaddition of allenenes bearing substituents on the allene moiety were also investigated. Monosubstitution at the proximal and distal carbons in allenes $151 \mathrm{~g}$ and $151 \mathrm{i}$ was tolerated and the

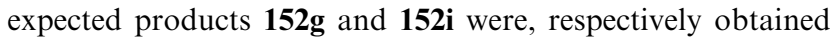
in high yields $(90-94 \%)$. On the other hand, decreased reactivity toward the cycloaddition was observed with allenenes $151 \mathrm{~h}$ and $151 \mathrm{j}$ bearing a geminal dimethyl group at the terminal position, leading to cycloadducts $152 \mathrm{~h}(46 \% ; 3: 1$ mixture of diastereomers) and $\mathbf{1 5 2} \mathbf{j}(63 \%)$ in longer reaction time (Scheme 48).

The stereoselective formation of cycloadducts $\mathbf{1 5 2}$ from terminal allenes $\mathbf{1 5 1}$ is illustrated in Scheme 49. Among the two conformations, $\mathbf{1 5 3}$ and $\mathbf{1 5 4}$ of the first cyclization, $\mathbf{1 5 4}$ would be destabilized by unfavourable steric interaction between the methylene protons of the terminal allenic carbon and the $\mathrm{R}^{\prime}$ group of the terminal olefin. Accordingly, the first cyclization preferentially would occur through 153 to give a diradical intermediate 155. Since the geometry of the olefin is completely preserved to yield the cycloadduct $\mathbf{1 5 2}$ without formation of 156, the second cyclization would involve a rapid ring closure before the bond rotation can occur. The fact that diastereomer $\mathbf{1 5 7}$ derived from the relatively unstable conformer $\mathbf{1 5 4}$ was not detected from terminal allenes would suggest that the energy difference between the two conformers 153 and 154 is significant in the case of terminal allenes.

On the other hand, the substitution on the terminal alkyne moiety is essential for the [2+2] cycloaddition of allenynes, providing the corresponding distal adducts with complete regioselectivity. Ohno and Tanaka, have investigated the thermal cycloaddition of allenynes $\mathbf{1 5 8}$ and the desired bicyclic compounds 159 were produced in good yields (Scheme 50). ${ }^{50}$ It has been observed that the effect of electron-donating and electron-withdrawing substituents on the aryl group is not dramatic. Likewise as in the reaction of allenenes, the $\alpha$-substituent of the allene moiety has proven to be significantly 


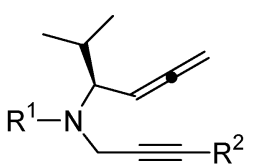

158 $\stackrel{\text { a) }}{\longrightarrow}$<smiles>[R]C1=C2CN([R7])[C@H](C(C)C)C=C2C1</smiles>

$159(61-94 \%)$

$\mathrm{R}^{1}=\mathrm{Mts}, \mathrm{Ts}$

$\mathrm{R}^{2}=\mathrm{Ph}, 4-\mathrm{Me}^{-} \mathrm{C}_{6} \mathrm{H}_{4}, 4-\mathrm{MeOC}_{6} \mathrm{H}_{4}$,

4- $\mathrm{CO}_{2} \mathrm{Me}-\mathrm{C}_{6} \mathrm{H}_{4}$

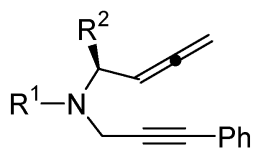

160
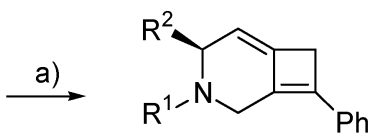

$161(75-94 \%)$

$\mathrm{R}^{1}=\mathrm{Mts}, \mathrm{Ts} ; \mathrm{R}^{2}=\mathrm{H}, \mathrm{Me}, \mathrm{Bn}$

Scheme 50 Intramolecular [2+2] cycloaddition reaction of allenynes 158 and 160. Reagents and conditions: (a) Dioxane, reflux, for 158: 2-3 h; for 160: 8-117 h.

important. For instance, the reaction of allenynes 160 bearing smaller substituents at the $\alpha$-position required longer reaction times $(8-117 \mathrm{~h})$.

Mukai and coworkers have examined the thermal [2+2] cycloaddition of allenynes 162 and 163 affording bicyclo[4.2.0] and [5.2.0] distal adducts as the only isomeric products (Scheme 51). ${ }^{51}$ Allenynes 162 and 163 were cleanly converted to bicyclo[4.2.0]octa-1,6-dienes 164 and

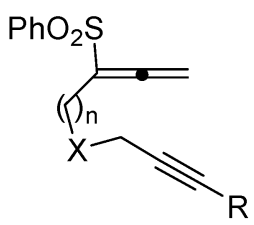

$162(n=1)$

$\mathrm{R}=\mathrm{Me}, \mathrm{nPr}, \mathrm{nBu}, \mathrm{Ph}, \mathrm{TMS}$

$X=O, N T s$

$163(\mathrm{n}=2)$

$\mathrm{R}=\mathrm{Me}, \mathrm{Ph}, \mathrm{TMS}$

$\mathrm{X}=\mathrm{O}, \mathrm{NTS}$
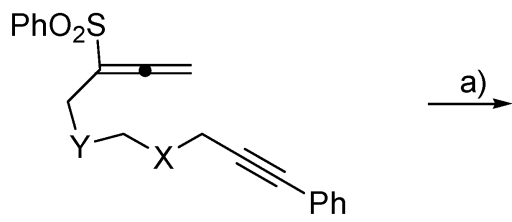

167

$\mathrm{X}=\mathrm{O}, \mathrm{CH}_{2}, \mathrm{NTs}$,

$\mathrm{Y}=\mathrm{CH}_{2}, \mathrm{C}\left(\mathrm{CO}_{2} \mathrm{Me}\right)_{2}$

Scheme 51 Intramolecular [2+2] cycloaddition of allenynes 162, 163 and $\mathbf{1 6 7}$ having a phenylsulfonyl functionality on the allenyl group. Reagents and conditions: (a) Xylenes or mesitylene, reflux, $\mathrm{N}_{2}, 7-30 \mathrm{~h}$.

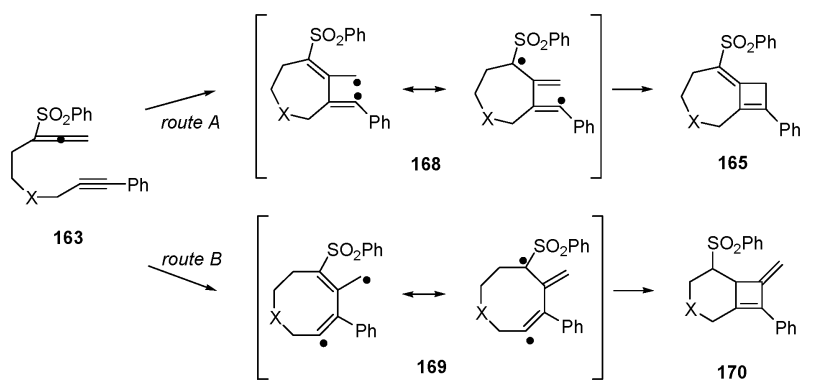

Scheme 52 Mechanism for the regioselective formation of the [m.2.0] frameworks through intramolecular [2+2] cycloaddition of allenynes. ${ }^{51}$

bicyclo[5.2.0]nona-1,7-dienes 165, respectively, in high yields. Furthermore, this method has been shown to be applicable to the construction of bicyclo[6.2.0]deca-1,8-dienes 166 from homologous allenynes $\mathbf{1 6 7}$.

The mechanism for the regioselective formation of the bicyclo[m.2.0] frameworks has been rationalized by invoking diradical intermediates (Scheme 52). For the thermal reaction of $\mathbf{1 6 3}$, for instance, there are two possible pathways. Route $A$ should involve allyl radical intermediate $\mathbf{1 6 8}$ formed by initial combination between the sp-hybridized carbon center of the allenyl moiety and the internal alkyne carbon. The resulting seven-membered diradical intermediate, having an allyl as well as phenylvinyl radical species, would immediately collapse into compound 165. An alternative initial connection of the sp-hybridized carbon of the allenyl moiety with the external alkyne carbon might lead to the eight membered intermediate 169 containing allyl and vinyl radicals. The ring-closing reaction of these intermediates would give rise to the production of compound 170 (route $B$, Scheme 52). The fact that compound 163 exclusively gave 165 in a high yield would strongly reflect the preferential formation of radicals 168 over 169 due to easier formation. The latter diradical species are eight-membered ring systems, while the former are seven-membered cycles. Similarly, the exclusive formation of compounds $\mathbf{1 6 4}$ and $\mathbf{1 6 6}$ may be rationalized by considering the ring size of the diradical species (6-membered versus 7-membered rings and 8-membered versus 9-membered rings).

Very recently Ma has reported an efficient synthesis of 3-oxabicyclo[4.2.0]octa-1(8),5-dien-4-ones $\mathbf{1 7 1}$ via the intramolecular [2+2] cycloaddition of propargylic 2,3-allenoates 172 under conventional heating conditions (Scheme 53). ${ }^{52}$ The authors have found that when $\mathrm{R}^{1}$ is an aryl group and $\mathrm{R}^{3}$ is a phenyl group, Diels-Alder aromatization adducts 173 were
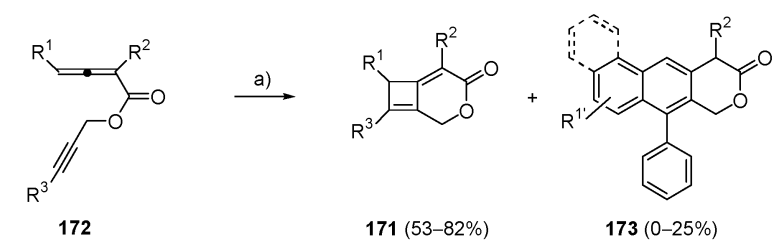

$\mathrm{R}^{1}=\mathrm{Ph}$, naphthyl

$\mathrm{R}^{2}=\mathrm{Me}, \mathrm{Et}, \mathrm{nPr}, \mathrm{nBu}, \mathrm{TMS}, \mathrm{Bn}$

$\mathrm{R}^{3}=\mathrm{Ph}, \mathrm{TMS}$

Scheme $53[2+2]$ Cycloaddition of propargylic 2,3-allenoates 172. Reagents and conditions: (a) Toluene, reflux, 8-72 h. 
formed as by-products in yields ranging from 5 to $25 \%$. However, when $\mathrm{R}^{3}$ is TMS, no Diels-Alder aromatization adducts were formed. Furthermore, when $\mathrm{R}^{1}$ is an alkyl group, the reactions take longer time with lower yields. However, $\mathrm{R}^{2}$ can be $\mathrm{H}$, alkyl, benzyl, and allyl groups. A dramatic alkynic substituent effect on the selectivity of the reaction has been observed: when $\mathrm{R}^{3}$ is TMS, the [2+2] cycloaddition reaction of propargylic 2,3-allenoates proceeded smoothly, probably due to their stabilization effect for the possible diradical intermediates involved in the reaction. The regioselectivity observed in the [2+2] cycloaddition of propargylic 2,3-allenoates 172 could be explained via formation of diradical species intermediates, analogously as it has been illustrated in Scheme 52 for allenynes $\mathbf{1 6 3}$.

In 2005, Brummond and co-workers reported that the microwave irradiation of alkynyl allenes 174 and 175 provides an efficient route to bicyclo[4.2.0]octadienes and bicyclo[5.2.0]nonadiene $\mathbf{1 7 6}$ and $\mathbf{1 7 7}$. The reaction takes place with complete regioselectivity for the distal double bond of the allene; thus selectively forming a variety of hetero- and carbocyclic substrates (Scheme 54). ${ }^{53}$ The authors have found that the optimum conditions for performing this allenic [2+2] cycloaddition reaction were a $3 \mathrm{M}$ solution of an ionic liquid,1-ethyl-3-methylimidazolium hexafluorophosphate, in toluene at $250{ }^{\circ} \mathrm{C}(\mathrm{MWI})$ for $15 \mathrm{~min}$. The scope of the reaction has been investigated by subjecting alkynyl allenes possessing a wide range of substituents and functionalities. The substitution on the terminal alkynes appeared to be a controlling factor in the efficiency of these reactions, with the phenyl group giving the highest conversions. Once again, the regioselectivity observed in compounds $\mathbf{1 7 4}$ and $\mathbf{1 7 5}$ is in agreement with previous results in related systems.

Independently, Oh's group has developed an efficient, simple and eco-friendly synthetic method for the preparation of fused bicyclic $[m, 2,0]$ alkadienes from 1,5-, 1,6- and 1,7allenyne carboxylates 178 and 179. ${ }^{54}$ Thus, compounds 178
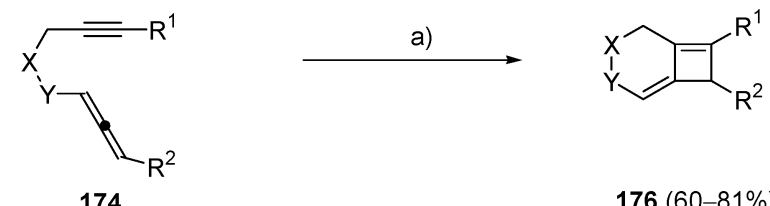

$\mathrm{X}=\mathrm{CH}_{2}, \mathrm{NBz} ; \mathrm{Y}=\mathrm{CH}_{2}, \mathrm{MeC}\left(\mathrm{CO}_{2} \mathrm{Me}\right)$

$176(60-81 \%)$

$\mathrm{R}^{1}=\mathrm{Me}, \mathrm{Ph} ; \mathrm{R}^{2}=\mathrm{H}, \mathrm{Me}$
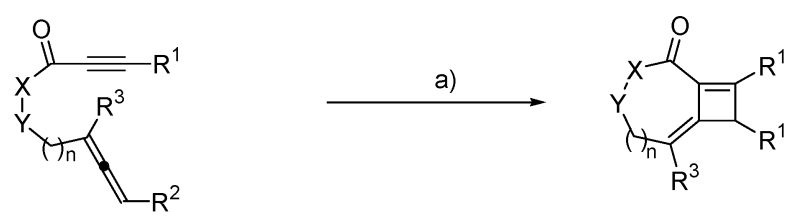

175

$\mathrm{n}=0,1 ; \mathrm{X}=\mathrm{NH}, \mathrm{CH}_{2} ; \mathrm{Y}=\mathrm{MeC}\left(\mathrm{CO}_{2} \mathrm{Me}\right), \mathrm{CH}_{2}$

$\mathrm{R}^{1}=\mathrm{Me}, \mathrm{Ph}, 4-\mathrm{MeO}-\mathrm{Ar} ; \mathrm{R}^{2}=\mathrm{Me} ; \mathrm{R}^{3}=\mathrm{H}, \mathrm{Me}$

$177(21-83 \%)$

Scheme 54 Microwave-assisted intramolecular [2+2] cycloaddition of allenynes 174 and 175. Reagents and conditions: (a) Toluene, ionic liquid (1-ethyl-3-methylimidazolium hexafluorophosphate), MWI at $250{ }^{\circ} \mathrm{C}, 15 \mathrm{~min}$.
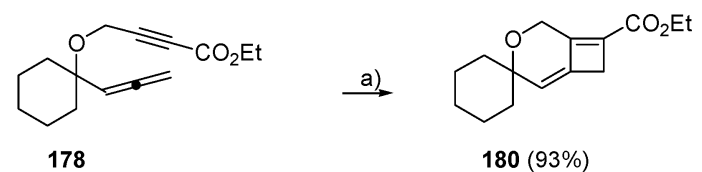

$180(93 \%)$
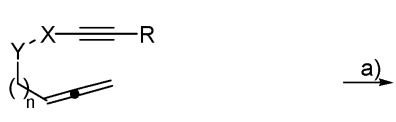

179b $n=0, X=\mathrm{CH}_{2} \mathrm{OH}, \mathrm{Y}=\mathrm{C}(\mathrm{Me})_{2}, \mathrm{R}=\mathrm{CO}_{2} \mathrm{Et}$

179c $\mathrm{n}=0, \mathrm{X}=\mathrm{CH}_{2} \mathrm{OH}, \mathrm{Y}=\mathrm{C}(\mathrm{Me})_{2}, \mathrm{R}=\mathrm{Ph}$

179d $n=2, X=\mathrm{CH}_{2}, Y=\mathrm{C}\left(\mathrm{CO}_{2} \mathrm{Et}\right)_{2}, \mathrm{R}=\mathrm{CO}_{2} \mathrm{Et}$

179e $\mathrm{n}=2, \mathrm{X}=\mathrm{CH}_{2}, \mathrm{Y}=p \mathrm{TsN}, \mathrm{R}=\mathrm{CO}_{2} \mathrm{Et}$

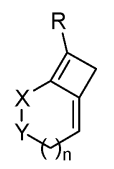

181b (97\%)

181c $(75 \%)$

181d $(79 \%)$

181e (78\%)
Scheme 55 Microwave irradiation of allenynes 178 and 179. Reagents and conditions: (a) Toluene, MWI, $70{ }^{\circ} \mathrm{C}, 10-45 \mathrm{~min}$.

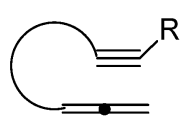

178, 179

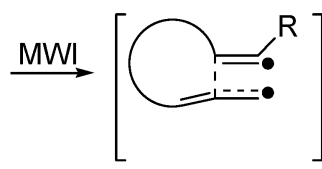

182

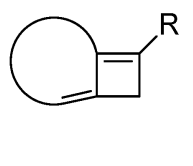

180,181
Scheme 56 Explanation for the synthesis of compounds 180 and 181 under microwave irradiation conditions. ${ }^{54}$

and $\mathbf{1 7 9}$ were cleanly converted into their corresponding distal [2+2] cycloadducts $\mathbf{1 8 0}$ and $\mathbf{1 8 1}$ under microwave irradiation (Scheme 55).

Scheme 56 shows a possible explanation for the formation of compounds 180 and 181. Microwave heating on substrates 178 and 179 might break one unsaturated bond to form a transient diradical such as $\mathbf{1 8 2}$, which would undergo rapid cyclization to give the adducts $\mathbf{1 8 0}$ and $\mathbf{1 8 1}$.

The microwave induced intramolecular allenyne $[2+2]$ cycloaddition of a variety of 1-allenyl-2-propargyl-substituted derivatives $\mathbf{1 8 3}$, as a synthetic route to the [5.6.4] ring core of sterpurene, has been reported in 2008 by Ovaska's group. Thus, irradiation of allenynes 183 smoothly afforded polycyclic compounds $\mathbf{1 8 4}$, in which the $\pi$-component was the external double bond of the allene moiety, as single isomers in excellent yields (70-92\%) (Scheme 57). ${ }^{55}$ The reaction has been shown to be rather general and independent of the type of substituent on the triple bond with the exception of terminal alkynes, which decomposed under the reaction conditions employed. Furthermore, only allenic systems having the $\mathrm{OH}$ and the propargylic moieties in cis configuration were found to be reactive under the above conditions.

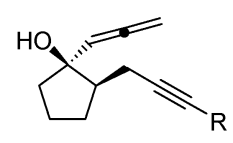

183

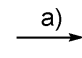

$\mathrm{R}=\mathrm{H}, \mathrm{Et}, \mathrm{Ph}, \mathrm{TMS}, \mathrm{TBS}, \mathrm{p}-\mathrm{TBSO}-\mathrm{C}_{6} \mathrm{H}_{4}$

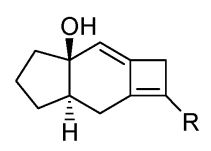

$184(0-92 \%)$
Scheme 57 Intramolecular [2+2] cycloaddition of 1-allenyl-2propargyl-substituted derivatives 183. Reagents and conditions: (a) Phenetole, MWI, $150-200{ }^{\circ} \mathrm{C}, 30-45 \mathrm{~min}$. 


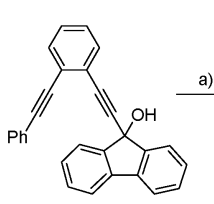

185

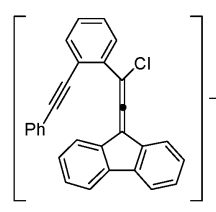

186

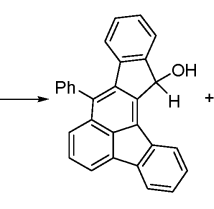

$187(74 \%)$

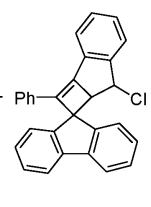

$188(12 \%)$
Scheme 58 Intramolecular [2+2] versus $[4+2]$ cycloadditions of benzoannulated allenyne 186. Reagents and conditions: (a) $\mathrm{Et}_{2} \mathrm{O}$, $\mathrm{SOCl}_{2}$, py, $0{ }^{\circ} \mathrm{C}$ to $\mathrm{RT}$.

On the other hand, bispropargylic alcohol $\mathbf{1 8 5}$ has shown to be an excellent precursor of allene 186. In this context, Wang and co-workers have investigated the competence of the [2+2] cycloaddition versus $[4+2]$ cycloaddition of allenes derived from bispropargylic alcohols under basic conditions. Thus, treatment of bisalkyne $\mathbf{1 8 5}$ with thionyl chloride in pyridine smoothly afforded adduct $\mathbf{1 8 7}$ in 74\% yield accompanied by a small amount of the $1 H$-cyclobut $[a]$ indene $188(12 \%)$, the $[2+2]$ cycloaddition product derived from the reaction of the allenic and the acetylenic moieties in intermediate 186 (Scheme 58). ${ }^{56}$

Surprisingly, treatment of bisalkynol 189 with thionyl chloride produced the $[2+2]$ cycloaddition adduct $\mathbf{1 9 0}$ exclusively in $81 \%$ yield (Scheme 59). This dramatic change of the reaction pathway has been attributed to the emergence of a steric strain due to the non bonded interactions with the chloro substituent along the pathway toward the formal Diels-Alder adduct. In fact, the importance of steric interactions in determining the reaction pathway has been supported by the monoprotected phenanthrenequinone examples. Thus, with a sterically more demanding, 5,5-dimethyl-1,3-dioxane protective group in 191, only the [2+2] cycloaddition adduct

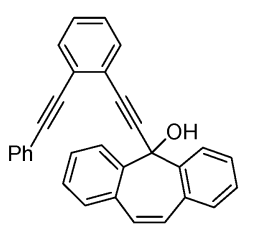

189

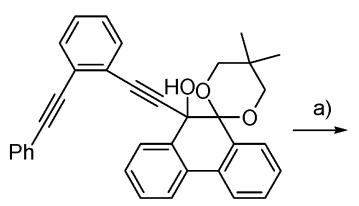

191

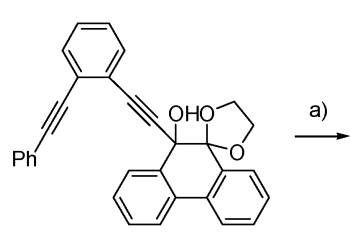

193

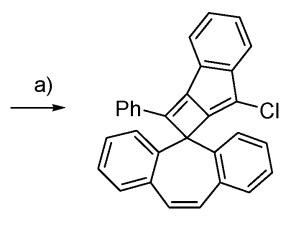

$190(81 \%)$

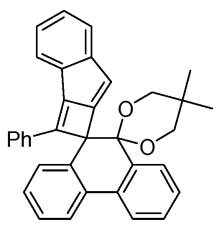

$192(80 \%)$

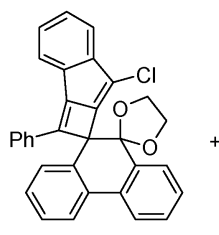

$194(34 \%)$

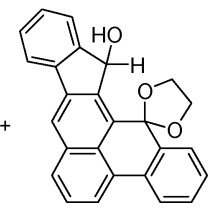

$195(35 \%)$
Scheme 59 Intramolecular $[2+2]$ versus $[4+2]$ cycloadditions of benzoannulated allenynes. Reagents and conditions: (a) $\mathrm{Et}_{2} \mathrm{O}, \mathrm{SOCl}_{2}$, py, $0{ }^{\circ} \mathrm{C}$ to $\mathrm{RT}$.

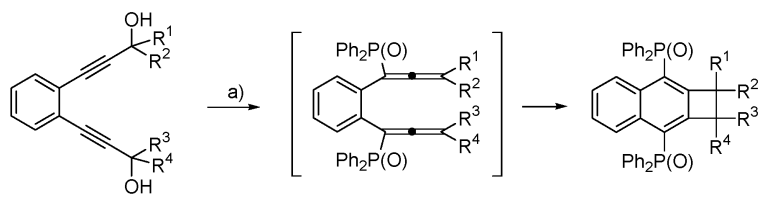

196

198

$197(84-90 \%)$

$\mathrm{R}^{1}=\mathrm{Me}, \mathrm{Ph}, \mathrm{CH}_{2} \mathrm{OBn} ; \mathrm{R}^{2}=\mathrm{H}, \mathrm{Me}$ $\mathrm{R}^{3}=\mathrm{H}, \mathrm{Ph} ; \mathrm{R}^{4}=\mathrm{H}, \mathrm{Ph}$

Scheme 60 [2+2] Cycloaddition reaction of bis(propargyl alcohols) 196 via transient bis(phosphinylallenes) 198. Reagents and conditions: (a) $\mathrm{Ph}_{2} \mathrm{PCl}, \mathrm{Et}_{3} \mathrm{~N}, \mathrm{THF}$, from $-78{ }^{\circ} \mathrm{C}$ to $\mathrm{RT}, 4 \mathrm{~h}$.

192 was also obtained. However, with a sterically less demanding ethylene ketal protective group in 193, a 1:1 mixture of the $[2+2]$ and the $[4+2]$ cycloaddition adducts 194 and 195 was isolated.

Later on, Kitagaki and Mukai reported that the reaction of benzene-bridged bis(propargyl alcohols) 196 with chlorodialkylphosphines exclusively afford 3,8-bis(dialkylphosphinyl)naphtho[ $b]$ cyclobutenes 197. Firstly, a dual [2,3]-sigmatropic rearrangement of the bisalkynols 196 gave intermediate 1,2-bis( $\alpha$-phosphinylallenyl)benzenes 198, which spontaneously undergo intramolecular [2+2] cycloaddition (Scheme 60). ${ }^{57}$ Compounds 197 were obtained in high yields in all cases.

Formation of compounds $\mathbf{1 9 7}$ has been rationalized via the intermediacy of diradical intermediates 199 as shown in Scheme 61. The bis(phosphinylallenes) 198, derived from the corresponding bis(propargylic phosphinites) by [2,3]-sigmatropic rearrangement, would be converted into the diradical species 199, which subsequently undergo intramolecular [2+2] cycloaddition to produce tricycles $\mathbf{1 9 7}$. The aromaticity of the final products is also a remarkable feature in the regiochemistry observed of the [2+2] cycloaddition, and will be the driving force to obtain compounds $\mathbf{1 9 7}$ as sole isomers.

Another example of competitive reaction involving the $[2+2]$ cycloaddition of allenynes was presented by Cook's group in 2003. During the synthesis of a dicyclopenta[a,e]pentalene via Pauson-Khand reaction, the monocyclized [5.4] system 200 was obtained upon heating the diyne-diallene 201 in toluene at $100{ }^{\circ} \mathrm{C}$ (Scheme 62). ${ }^{58}$

Ketene is also a suitable partner for the $[2+2]$ cycloaddition with allenes, though, only the intermolecular version has been published. ${ }^{59}$ However, Halcomb has reported the intramolecular $[2+2]$ cycloaddition of ketenes with allenes. Thermal intramolecular [2+2] cycloaddition between an in situ generated ketene

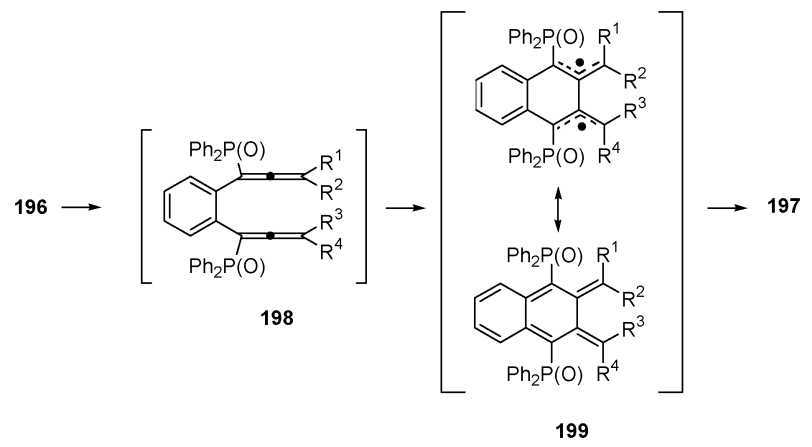

Scheme 61 Mechanistic explanation for the formation of adducts $197 .{ }^{57}$ 


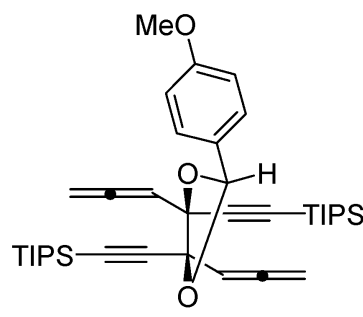

201

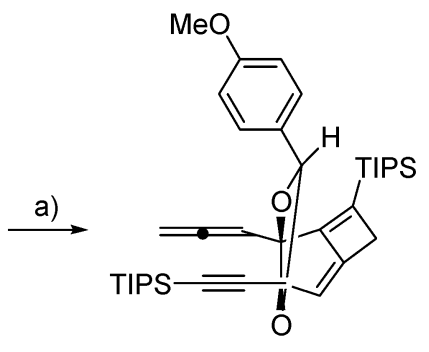

200
Scheme 62 Formation of the [2+2] adduct $\mathbf{2 0 0}$ when attempting the Pauson-Khand reaction of bis-allenyne 201. Reagents and conditions: (a) Toluene, $100{ }^{\circ} \mathrm{C}$.

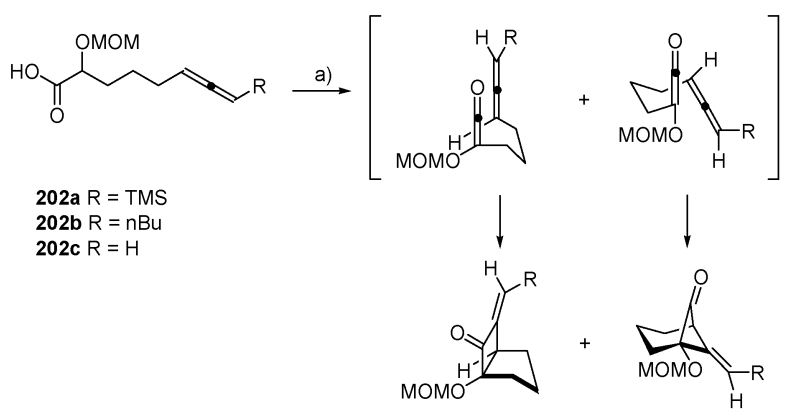

203a $E: Z=2.2: 1+204 a \quad E: Z=4: 1(1: 3.5,70 \%)$ 203b $E: Z=2: 1+204 b E: Z=1: 1.2(3: 1,78 \%)$ 203c + 204c $(5: 1,45 \%)$

Scheme 63 Intramolecular ketene-allene [2+2] cycloaddition. Reagents and conditions: (a) Oxalyl chloride (3 equiv.), $\mathrm{Et}_{3} \mathrm{~N}$ ( 2.5 equiv.), benzene, RT, $4-10 \mathrm{~h}$; then reflux, $12 \mathrm{~h}$.

and a 1,2-diene, in compounds 202, afforded 7-methylidinebicyclo[3.2.0] heptanones 203 and 7-methylidinebicyclo[3.1.1] heptanones 204 (Scheme 63). ${ }^{60}$ Compounds 203 arose from formation of a bond between the central ketene carbon and the central allene carbon to afford the [3.2.0] fused bicyclic system. However, the [3.1.1] bridged bicyclic systems 204 have resulted from bond formation involving the alkoxy-substituted carbon of the ketene and the internal allene carbon. The [3.2.0] bicyclic adduct has been obtained as the major isomer for $\mathbf{2 0 2} \mathbf{b}$ $(\mathrm{R}=\mathrm{nBu})$ and 202c $(\mathrm{R}=\mathrm{H})$. By contrast, the selectivity of the cycloaddition was different for 202a ( $R=T M S)$; the [3.1.1] bicycloheptanone 203a was obtained as the major product. Besides, the $E$-olefin isomer was generally the major geometric isomer observed. Interestingly, no products derived from the cycloaddition of the ketene with the distal allenic carbon were observed.

The preference for $E$-olefin isomer formation has been explained in terms of steric demand; the ketene would prefer to approach the internal $\pi$-bond of the allene from the face which is not blocked by the substituent on the distal carbon of the external $\pi$-bond.

\section{Photochemical $[2+2]$ cycloadditions}

The $[2+2]$ photocycloaddition reaction is one of the most frequently employed photochemical transformation allowing

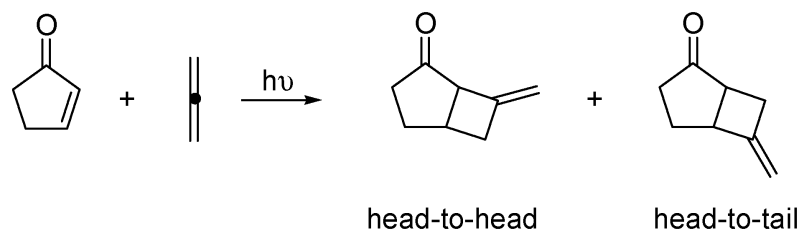

Scheme 64 Formation of the two possible regioisomers in the [2+2] photocycloaddition of 2-cyclopentenone and an allene.

the construction of the cyclobutane ring. ${ }^{61}$ In particular, this strategy is very important in the synthesis of natural products. ${ }^{62}$ In fact, it has been demonstrated that the intramolecular photocycloaddition of monosubstituted allenes with chiral enones is a useful strategy for the construction of carbon-carbon bonds in polycyclic structures. ${ }^{63}$ Wiesner has been the first to demonstrate the utility of the intramolecular diastereoselective allene-enone [2+2] cycloaddition in natural products synthesis. ${ }^{64}$ Later, Pirrung employed the intramolecular allene-enone cycloaddition in an elegant synthesis of pentalenolactone G. ${ }^{65}$ In addition, the photochemical additions to enones are believed to proceed through the intermediacy of a 1,4-diradical species which may revert to the starting allene and enone with loss of allene optical activity. In fact, Becker's studies as well as seminal contributions by Corey in related alkene-enone cycloadditions have provided a starting point from which to investigate asymmetric induction with optically active 1,3-disubstituted allenes.

The photochemical cycloaddition of allene to 2-cyclopentenone affording fused cyclobutane adducts with preferential head-to-head regiochemistry has been reported (Scheme 64). ${ }^{66}$ This result is in contrast to the preferential head-to-tail orientation of addition to cyclic enones observed with the majority of mono-substituted alkenes. ${ }^{67}$

The origin of the regioselectivity and the mechanism of the allene photocycloaddition reaction with cyclic enones have been the subject of debate. ${ }^{68}$ In particular, seminal papers of Kice and co-workers have contributed dealing with radical additions to allenes and have been applied to discuss the regiochemical outcome of $[2+2]$ cycloadditions of conjugated enones to allenes. ${ }^{69}$ By analogy to the corresponding reaction with simple alkenes it is assumed that the addition occurs from the enone triplet excited state and proceeds via triplet 1,4-diradical intermediates formed by bonding of an allene to either the 2-position or the 3-position of the enone. Scheme 65 shows the diradical intermediates formed. Diradicals 205 and 206 are the possible precursors of the head-to-tail cycloadduct, while diradicals $\mathbf{2 0 7}$ and $\mathbf{2 0 8}$ are the possible precursors of the head-to-head cycloadduct (Scheme 65).

Weedon, postulated that in the addition of allene to cyclopentenone, the relative amounts of the four triplet 1,4-diradicals could be determined by trapping experiments using $\mathrm{H}_{2} \mathrm{~S}{ }^{70}$ In these trapping experiments, diradical 205 formed $68 \%$ of the intermediates, while diradicals 207, 208 and 206 arose $29 \%, 3 \%$ and $0 \%$, respectively. ${ }^{71}$ This data indicates that addition at the $\alpha$-position of the enone is overwhelmingly favored. Also in contrast to the exciplex model which depends on the relative amounts of the various diradicals, the product distributions have been interpreted as depending on the competition of the particular diradicals between reversion 


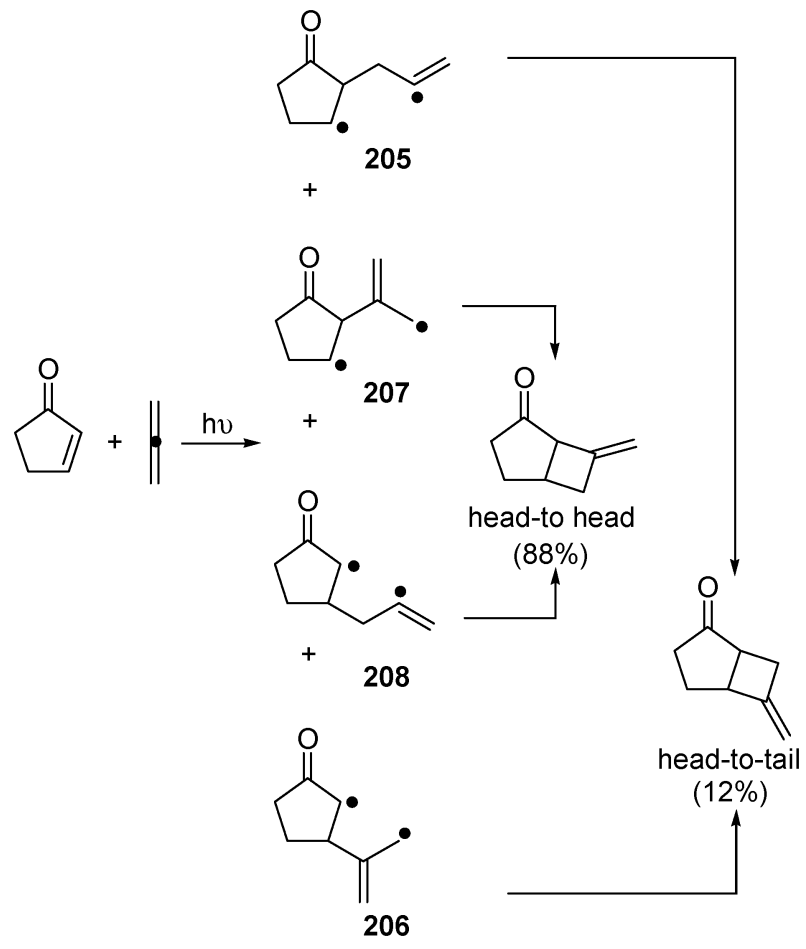

Scheme 65 Diradicals 205-208 formed in the intermolecular photocycloaddition of 2-cyclopentenone and an allene. ${ }^{69}$

to reactants and closure products. In particular, since the diradicals have nearly free rotation about the new single bond formed, the conformation of the triplet diradical upon spin inversion should be an important factor in determining whether the molecule will close to products or revert to starting materials.

In this context, theoretical studies of regioselectivity in the photochemical cycloaddition of allene to cyclopentenone, including energetics, conformations, spin-orbit coupling constants and the singlet-triplet energy splittings of the intermediate diradicals, have been reported by Goddard. ${ }^{72}$ This author postulated that the diradical containing the allyl fragment was predicted to be approximately $83 \mathrm{~kJ} \mathrm{~mol}^{-1}$ lower in energy than the other two. Using a model for the reaction mechanism in which the relaxed triplet excited state of allene reacts with ground state cyclopentenone, the reaction barriers to forming these allylic substituted systems are lower by $c a$. $33-42 \mathrm{~kJ} \mathrm{~mol}^{-1}$ in comparison to the other two. The conformation of the diradicals upon spin inversion is in all probability one important factor in determining whether the diradicals close to products or revert to starting materials. The $\mathrm{sp}^{3}$ hybridization at the bond-forming carbon in the vinyl (nonallylic) systems leads to nearly free rotation about that bond, and three shallow minima and the corresponding transition states for internal rotation were located. One minimum has the two carbon centers which carry the excess spin density about $3.8 \AA$ apart while in the other minima the distances are $\sim 3.1 \AA$. Taking these distances into account, it has been estimated that very few of the intermediates formed from the addition to the carbonyl close to products. In contrast, a high percentage of the systems formed from attack $\beta$ to the carbonyl are predicted to yield products. In the allylic substituted systems, carbon centers with some radical character are always relatively close

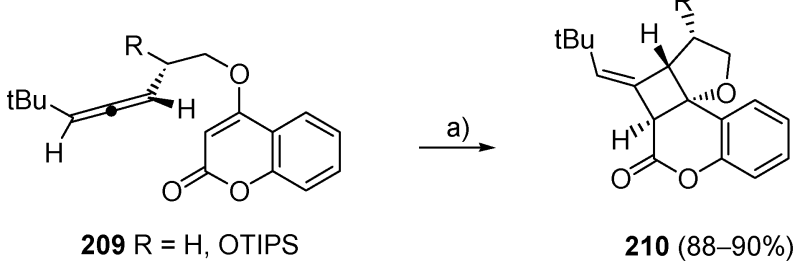

Scheme 66 Diastereoselective [2+2] photocycloaddition of allenic coumarins 209. Reagents and conditions: (a) hv, cyclohexane or dichloromethane, $23{ }^{\circ} \mathrm{C}, 4 \mathrm{~h}$.

to the site of radical character in the cyclopentenone ring. Thus, the relative ratio of cycloadducts can be estimated from the distribution of the diradicals. The transition state energies do not agree with the interpretations of experimental data by Weedon. The theoretical predictions suggest that the two allyl substituted systems should form most, if not all, of the 1,4diradical intermediates. In fact, it was predicted that the four 1,4-diradicals 205:207:208:206 would form in a ratio of $0: 83: 0: 17$. Taking to account that the arbitrarily relative efficiency of ring closure for 205, 206, 207 and 208 is, $10 \%$, $80 \%, 80 \%$ and $100 \%$, respectively, formation of head-to-head and head-to-tail adducts have been estimated to be $80: 20$.

A remarkable example was reported by Carreira in 1994, involving the photocycloaddition of optically active tert-butylsubstituted allenes with enones to give photoadducts in excellent yields and up to $99 \%$ asymmetric induction (Scheme 66). ${ }^{73}$ Thus, [2+2] photocycloaddition of allenic coumarins 209 cleanly afforded tetracycles 210 in excellent yields.

The high levels of asymmetric induction were rationalized according to the accepted model proposed by Becker for intramolecular allene photocycloadditions. ${ }^{74}$ The triplet enone $\mathrm{C}_{\beta}$ adds to the less hindered end of the allene $\mathrm{C}^{\prime}$, opposite to the tert-butyl substituent, to give a 1,4-diradical intermediate 211 which undergoes spin inversion and collapses to the exomethylenecyclobutanes 212 (Scheme 67). The observation of high enone face selectivity is accommodated by exo-approach of the allene onto the enone such that $\mathrm{H}^{\prime}{ }^{\prime}$ and not $\mathrm{C}^{\prime}$ resides over the ring. The high levels of asymmetric induction in the photoadducts are consistent with the observation that the configuration of the newly formed vicinal stereocenters is established kinetically upon formation of the $\mathrm{C}_{\beta}-\mathrm{C}^{\prime}$ bond and that for 209 reversion does not occur competitively with ring closure $\left(k_{\mathrm{c}} \gg k_{\mathrm{r}}\right)$. However, subtle conformational effects can effect the relative rates of closure, $k_{\mathrm{c}}$, and reversion, $k_{\mathrm{r}}$ (Scheme 67). In addition to proceeding with high levels of

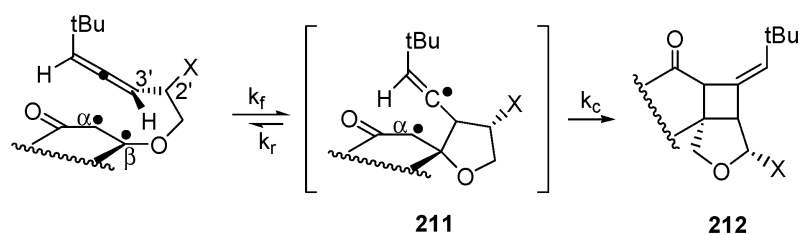

Scheme 67 Rationalization of the high levels of asymmetric induction on the intramolecular [2+2] photocycloaddition of allenic coumarins $209 .^{74}$ 


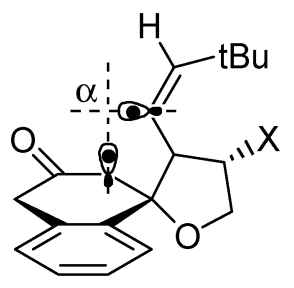

211

Fig. 7 Proposed transition-state for the formation of compounds $\mathbf{2 1 0}$ illustrating the optimal geometry of the 1,4-diradical $\mathbf{2 1 1}$ formed.

enantioselectivity, the [2+2] cycloaddition of allenic coumarins 209 affords in each case a single olefin diastereomer.

The formation of a single alkene may result from conformational memory effects in the 1,4-diradical intermediate 211 as it undergoes intersystem crossing and ring closure (Fig. 7). It has been proposed that in the coumarin-derived 1,4-diradicals, the orbitals are disposed in an arrangement with an interorbital angle close to $90^{\circ}$. Thus, the formation of a single olefin diastereomer from 209 is a consequence of two rapid, successive processes, intersystem crossing and ring closure, which allow the initial orientation of the tert-butyl substituent on the allene to be reflected in the stereochemistry of the products.

Later on, the same authors demonstrated the synthetic utility of the allene-enone [2+2] photocycloaddition by incorporation of a removable silane group onto the allenyl moiety. ${ }^{75}$ In analogy to the reported investigation involving tert-butyl-substituted allenes, the coumarin-derived adducts 212 were obtained as single alkene diastereomers starting from allenic coumarins 213 (Scheme 68).

More recently, Winkler and co-workers have reported the intramolecular photoaddition of vinylogous allenamide-type compounds 214, as an approach to the synthesis of pyrroles and furans 215 in moderate to good yields (31-87\%) (Scheme 69). ${ }^{76}$ Formation of pyrroles and furans 215 takes place from the corresponding cyclobutane photoadducts $\mathbf{2 1 6}$ which have been obtained via the [2+2] cycloaddition of the starting allenamides 214 .

The regiochemical outcome of the cycloaddition ring closure depends mainly on the presence or absence of an electron-withdrawing group at the nitrogen. In fact, when the substituent is $\mathrm{H}$ or $\mathrm{Me}$, products derived from parallel cycloaddition of the terminal allenic double bond to the olefin via $\mathbf{2 1 7}$ were observed. However, irradiation of vinylogous amide or imide $(\mathrm{R}=\mathrm{Ac})$, via 218 and 219, has lead to the selective formation of crossed photoadduct $\mathbf{2 2 0}$ (Scheme 70).
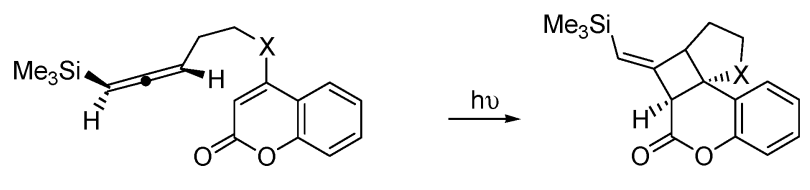

$213 X=0, S$

$212(80-90 \%)$

Scheme $68[2+2]$ Photocycloadditions of allenic coumarins 213. Reagents and conditions: (a) $h v$, cyclohexane or dichloromethane, $23{ }^{\circ} \mathrm{C}, 1.5 \mathrm{~h}$.

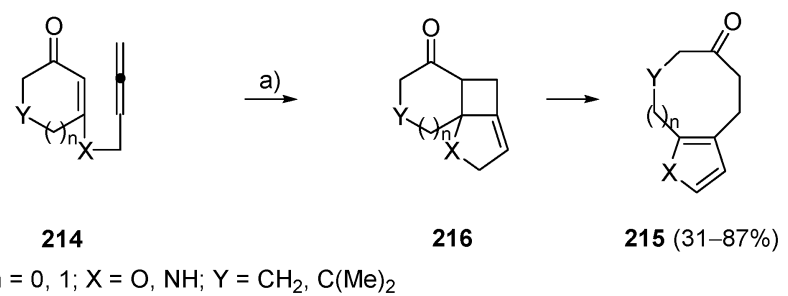

Scheme 69 Synthesis of pyrroles and furans via intramolecular [2+2] photocycloaddition of allenamide-type compounds 214. Reagents and conditions: (a) hv, $1.0 \mathrm{mM}, \mathrm{MeCN}$, pyrex photoreactor, $30 \mathrm{~min}$.

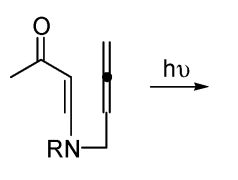

$214(\mathrm{R}=\mathrm{H}, \mathrm{Me})$

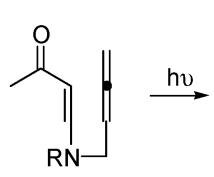

$214(R=A c)$

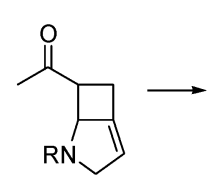

216

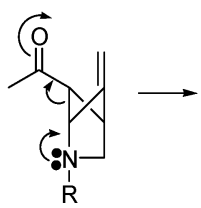

218

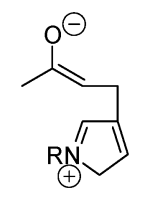

217<smiles>CC(=O)C=CCC(C)O</smiles>

219

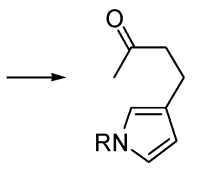

215

Scheme 70 Regiochemical outcome of the photochemical ring closure of allenamides 214 . $^{76}$

The photochemistry of divinylmethanes ${ }^{77}$ in which one vinyl moiety is an $\alpha, \beta$-unsaturated ester ${ }^{78}$ or an ylidenemalononitrile ${ }^{79}$ has been found to undergo regioselective di- $\pi$-methane rearrangement by multiplicity of the excited state. Interest on the photochemoselectivity by multiplicity control has been demonstrated in the photochemistry of $\beta$-allenyl-substituted alkylidenemalonates and $\alpha, \beta$-unsaturated esters affording the intramolecular [2+2] cycloaddition predominantly under direct and sensitized photolyses. ${ }^{80,81}$

In this context, Tsuno reported that allenyl(vinyl)methanes 221 and 222 have a triplet excited state of the allene or vinyl moiety and their states produces a cross-conjugated triene from the excited state of the allenyl chromophore and an intramolecular [2+2] cycloadduct from the vinyl chromophore. ${ }^{82}$ The direct photolysis of the diester 221a in hexane gave a cross-conjugated triene 223a, bicyclo[2.1.0]pentane 224a, pyran 225a and cyclopropene 226a (Scheme 71). ${ }^{83}$ On the other hand, the direct photolysis of the monoester 223b afforded adducts 223b, 224b, and 226b (Scheme 71). Irradiation of the isolated [2+2] cycloadducts 224 afforded cyclopropenes 226. Though the acetone-sensitized photolyses of $\mathbf{2 2 1}$ afforded triene 223, and the [2+2] cycloaddition products $\mathbf{2 2 4}$, polymeric materials were significantly produced. From the results obtained, it has been reported that acetone and acetophenone are good sensitizers for the isomerization to the trienes $\mathbf{2 2 3}$ and to the [2+2] cycloadducts $\mathbf{2 2 4}$.

The direct photolysis of 222a in acetonitrile gave mainly cross-conjugated triene 227a and bicyclo[2.1.0]pentane 228a. In the case of acetone-sensitization of 222a, the bicyclo[2.1.0]pentane 228a and indene 229a were obtained. In addition, 
<smiles>[R]C(=CC(=C(C)C)C(C(C)=O)=C(C)C)c1ccccc1</smiles>

223a (4\%)

223b (1\%)<smiles>[R]C(=CC(C)(C)C(=CC(C)C)c1ccccc1)C(C)C</smiles>
$\stackrel{\text { a) }}{\longrightarrow}$

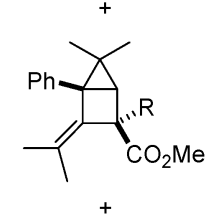

224a (15\%) 224b $(25 \%)$ 221a $\mathrm{R}=\mathrm{CO}_{2} \mathrm{Me}$
221b $\mathrm{R}=\mathrm{H}$ 225a (18\%) 225b $(0 \%)$

226a (4\%) 226b (15\%)

Scheme 71 Direct photolysis reaction of allenenic esters 221. Reagents and conditions: (a) hv, hexane, RT, 25-33 h.

acetone, acetophenone, benzophenone and thioxanthen-9-one have shown sensitizing effects for the formation of $\mathbf{2 2 7}$ and $\mathbf{2 2 8}$ (Scheme 72).

The direct photolysis of 222b in acetonitrile afforded and allenyl cyclopropane 230b, bicyclo[2.1.0]pentane 228b, and

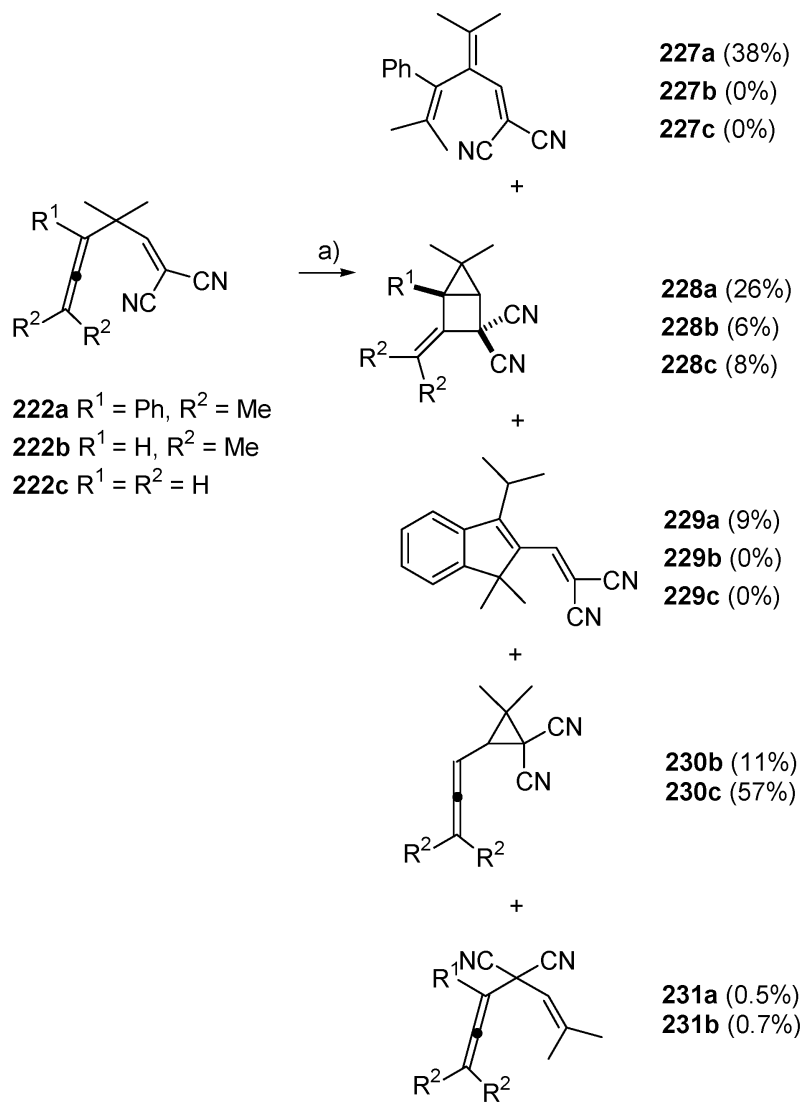

Scheme 72 Direct photolysis reaction of allenenic dinitriles 222 . Reagents and conditions: (a) hv, MeCN, RT, 5-55 h.

1,3-phenyl rearrangement compound $\mathbf{2 3 1 \mathbf { b }}$ as intramolecular photoproducts and further led to considerably polymeric compounds and small amounts of unidentified intramolecular compounds. On the other hand, the dinitrile 222c under direct photolysis underwent mainly a di- $\pi$-methane rearrangement product 230c and the intramolecular [2+2] cycloadduct 228 was obtained as a by-product. However, the acetone-sensitization of 222b or 222c gave the intramolecular [2+2] cycloadduct $228 \mathbf{b}$ or $228 \mathrm{c}$ in better yields ( 53 and $59 \%$, respectively).

The sensitizing effects of esters 221a and 221b and nitrile 222a have suggested that the trienes and [2+2] cycloadducts are derived from the triplet excited state of a different chromophore in the substrates. The triplet energy required for the formation of the [2+2] cycloadduct from ester 221a or 221b is estimated to be $c a .300 \mathrm{~kJ} \mathrm{~mol}^{-1}$, while that from the nitrile 222a is $c a .250 \mathrm{~kJ} \mathrm{~mol}^{-1}$. These results are in agreement with previous reports of the photochemistry on the $\gamma$-allenylsubstituted $\alpha, \beta$-unsaturated esters, where the triplet energy required for the formation of the [2+2] cycloadduct is estimated to be $c a .300 \mathrm{~kJ} \mathrm{~mol}^{-1}$ and the activated chromophore is the vinyl moiety. ${ }^{84}$ Furthermore, the triplet energy of the ylidenemalonitrile has been reported to be $c a .250 \mathrm{~kJ} \mathrm{~mol}^{-1}$. The reported triplet energy corresponds with the estimated values for 222a. Hence, the intramolecular [2+2] cycloaddition of $\mathbf{2 2 1}$ and 222a under the sensitized photolyses occurs from the triplet of the vinyl chromophore. On the other hand, the triplet energy required for the formation of trienes 223 or 230a from 221 and 222a is estimated to be $c a .260 \mathrm{~kJ} \mathrm{~mol}^{-1}$. It has been suggested that the triplet energy of monophenyl-substituted allene lies in the range of $250-270 \mathrm{~kJ} \mathrm{~mol}^{-1}$. Because this range includes the estimated triplet energy of the phenyl-substituted allenyl chromophore for esters 221a and 221b, acetone and acetophenone having higher triplet energy competitively affect both chromophores in 221a and 221b, although the sensitizers such as benzophenone, 2-benzoylpyridine and thioxanthen-9one in the range of $260-290 \mathrm{~kJ} \mathrm{~mol}^{-1}$ can reach the excited state for the allenyl chromophore. The reaction mechanism of the intramolecular [2+2] cycloaddition of the allenyl(vinyl)methanes by trapping experiments of a diradical intermediate using hydrogen selenide has been proved ${ }^{81}$ Thus, the excited state of the vinyl chromophore in $\mathbf{2 2 1}$ and 222a initially bonds between $\mathrm{C} 2$ and $\mathrm{C} 6$ to afford a cyclopenta-1,3-diyl radical $\mathbf{2 3 2}$ or $\mathbf{2 3 3}$ from which ring closure yields the intramolecular [2+2] cycloadducts (Scheme 73 and 74). On the other hand, for the formation of the trienes, it has been considered that the excited state of the allenyl chromophore bonds between $\mathrm{C} 3$ and $\mathrm{C} 5$ to yield 234 and 235, which undergoes a 1,2-phenyl migration to yield the trienes. This reaction pathway have been supported by PM3 MO calculation, where the optimal structure of diradical 236 corresponds to the structure of 223b. 2-Methyl1,3-butadiene under direct photolyses has been recognized to have a quenching effect on the formation of the $[2+2]$ cycloadducts. The intramolecular [2+2] cycloaddition of the allenyl(vinyl) methanes occurs via both the singlet and triplet pathways, in the cases of the photochemistry of 221. Hence, due to the quenching effects, the trienes are derived via the triplet excited state.

The direct photolysis of 221a gave pyran 225a, which was not obtained from sensitised photolyses and its formation did 


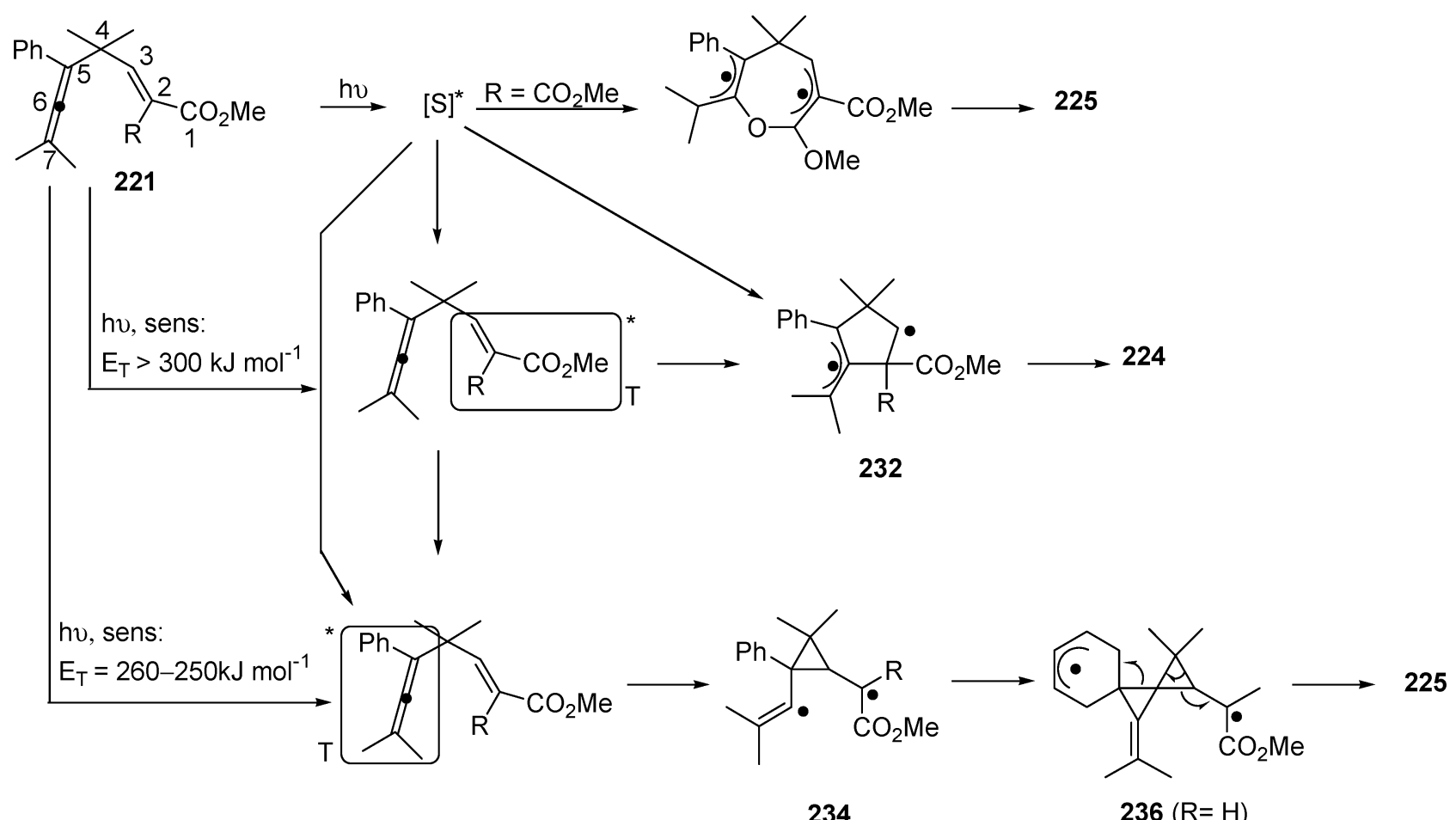

Scheme 73 Reaction mechanism for the photochemistry of esters $221 .{ }^{82,83}$

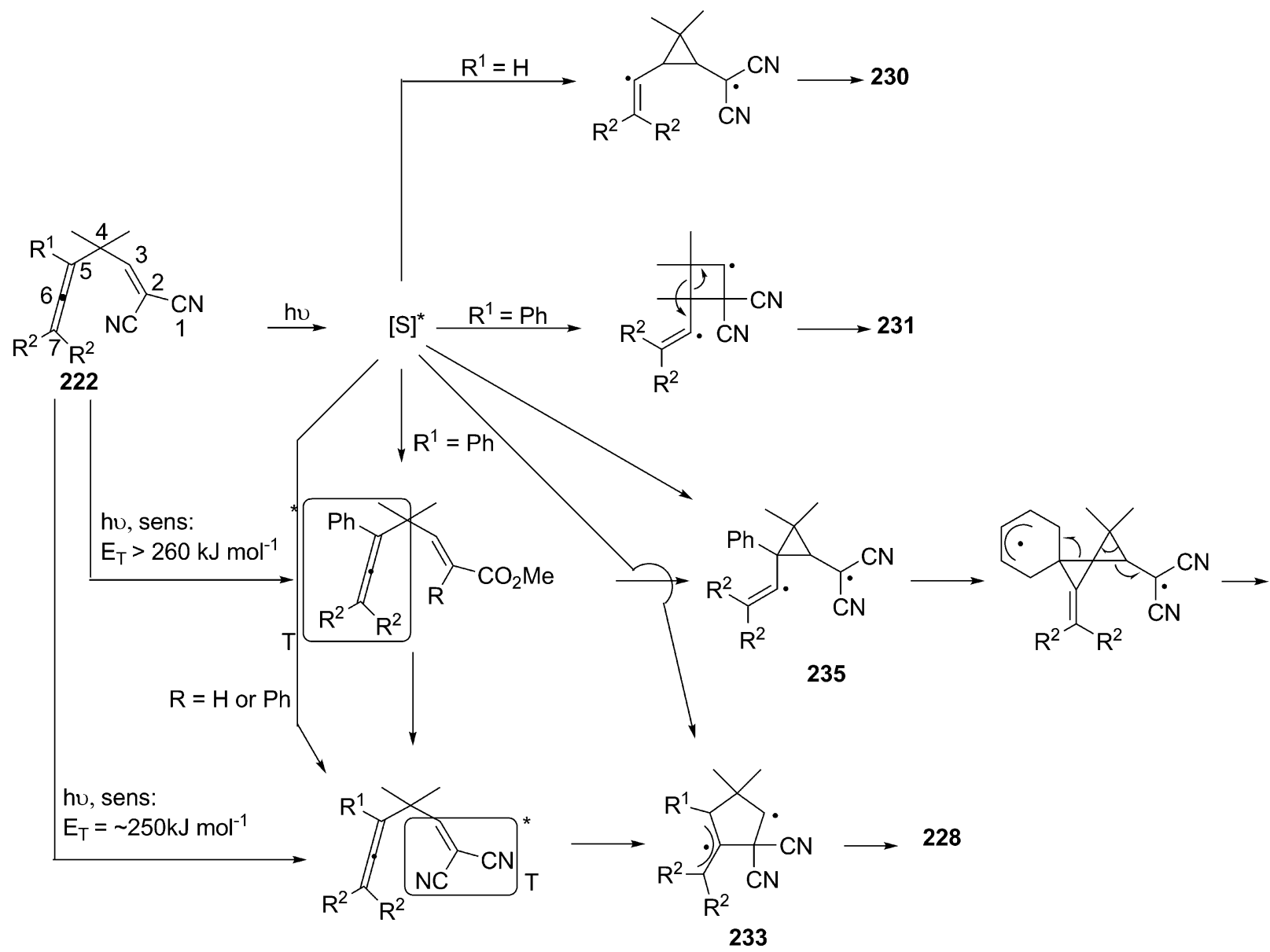

230

Scheme 74 Reaction mechanism for the photochemistry of nitriles $\mathbf{2 2 2} .^{82,83}$ 
not show the quenching effect by 2-methyl-1,3-butadiene. Therefore, 225a is derived from the photochemical intramolecular oxa-Diels-Alder reaction via singlet-excited state of a $Z$-carbonyl in 221a (Scheme 73).

The direct photolysis of 222b and 222c gave allenylcyclopropanes $230 \mathbf{b}$ and 230 c, i.e. di- $\pi$-methane rearrangement compounds, while the diesters which have the same allenyl chromophore in 222b and 222c led mainly to the [2+2] cycloadduts. Such a difference has been explained by the MO coefficients of the vinyl chromophore. The MO coefficient for the $\alpha$-carbon of the 2,2-dimethylpropylidenemalonate is larger than that of the $\beta$-one. Hence, $\gamma$-allenylsubstituted $\alpha, \beta$-unsaturated esters undergo preferential interaction between the allene center and the $\alpha$-carbon. However, the PM3 MO calculation for 2,2-dimethylpropylidenemalononitrile as a model of the vinyl moiety in $\mathbf{2 2 2}$ indicates that the MO coefficient $(0.68)$ of the $\beta$-carbon is larger than that $(0.50)$ of the $\alpha$-carbon. In the photochemistry of $222 \mathbf{b}$ and $222 \mathbf{c}$, therefore, the di- $\pi$-methane rearrangement occurred by preferential MO interaction between the C5 and C3 (Scheme 74). On the other hand, it seems that the minor compounds 231 were produced by the MO interaction between $\mathrm{C} 5$ and $\mathrm{C} 2$ in the nitriles $\mathbf{2 2 2} \mathbf{b}$ and 222c (Scheme 74).

Apparently, cyclopropenes $\mathbf{2 2 6}$ are derived via the photochemical pathway of the [2+2] cycloadducts 224 (Scheme 75).

On the basis of the photochemistry of $\gamma$-allenyl-substituted $\alpha, \beta$-unsaturated esters and nitriles, it has been shown: (a) Due to the HOMO of a 1,2-propadienyl group lies at the $\mathrm{C} 2-\mathrm{C} 3$ moiety and the MO coefficients of $\mathrm{C} 3$ is higher value than that of the $\mathrm{C} 2$, the 1,2-propadienyl substituted esters undergo more ineffective intramolecular $[2+2]$ cycloaddition than the 3-methyl-1,2-propadienyl-substituted esters; (b) The allenyl(vinyl)methanes initially bond between the allene center carbon and the $\alpha$-carbon of the $\alpha, \beta$-unsaturated ester or the nitrile moiety to give cyclopentane-1,3-dienyl radicals; (c) The intramolecular [2+2] cycloaddition occurs via both singlet and triplet excited states; (d) The triplet intramolecular [2+2] cycloaddition occurs from the excited state of the $\alpha, \beta$-unsaturated ester or the nitrile moiety.

Later on, the photolyses of $\gamma$-allenyl-substituted alkylidenecycloalkanones 237 gave [2+2] cycloadducts 238 and 239 in very low yields along with $(Z)$-form $\mathbf{2 4 0}$ and pyran 241. Analogously, $[2+2]$ cycloaddition of $\gamma$-(3-methyl-1,2butadienyl)-substituted 3-alkylidenepenta-2,4-diones $\mathbf{2 4 2}$ afforded

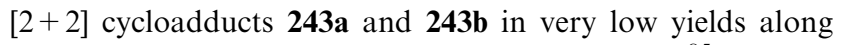
with compounds $\mathbf{2 4 4}$ and $\mathbf{2 4 5}$ (Scheme 76 and 77). ${ }^{85}$

Analogous tendence to the photochemistry of $\gamma$-allenylsubstituted $\alpha, \beta$-unsaturated esters were observed. ${ }^{80,84}$ Thus, it is obvious that the HOMO in the allenyl moiety contributes greatly to the photochemical intramolecular $[2+2]$ cycloaddition

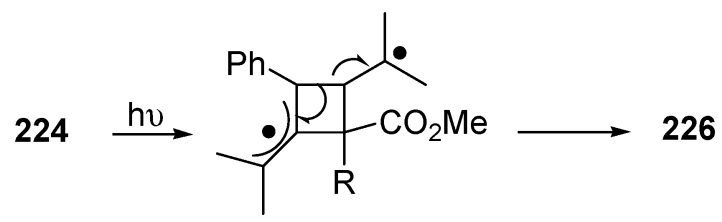

Scheme 75 Formation of cyclopropanes 226 from [2+2] cycloadducts 226.

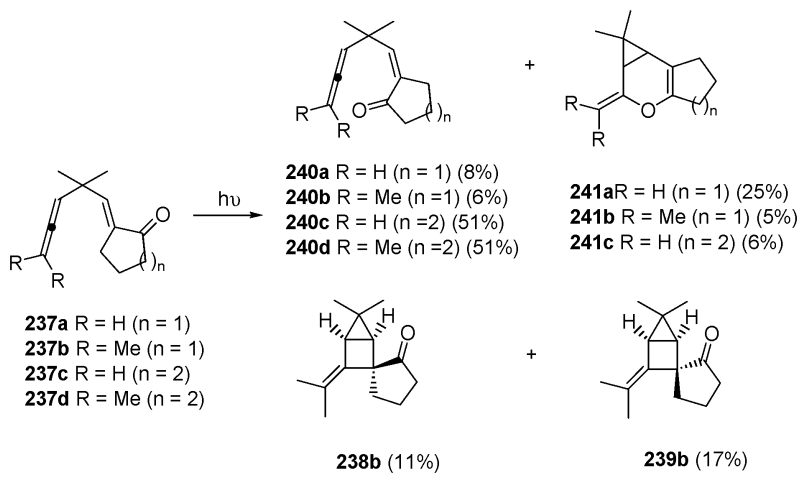

Scheme 76 Photochemical reaction of allenenic ketones 237. Reagents and conditions: (a) $h v$, hexane, RT, 9h.

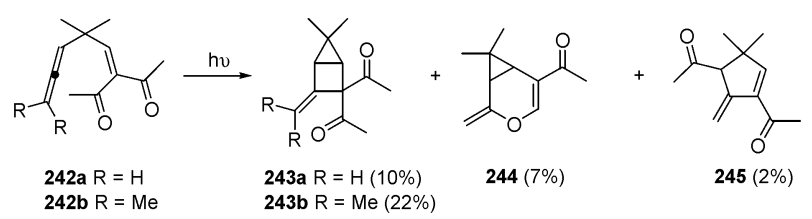

Scheme 77 Photochemical reaction of allenenic diketones 242 Reagents and conditions: (a) $h v$, hexane, RT, $9 \mathrm{~h}$.

of these enones. The triplet energies of the enones 240a and 240b are $c a$. $280 \mathrm{~kJ} \mathrm{~mol}^{-1}$, and futher, those of the diketones 242a and 242b are estimated to be in range of $280-260 \mathrm{~kJ} \mathrm{~mol}^{-1}$. These estimated triplet energies are close to the reported triplet energies of the $\alpha, \beta$-unsaturated enones. Therefore, the intramolecular $[2+2]$ cycloaddition by the triplet sensitization occurs from the triplet excited state of the enone moiety. The MO coefficients obtained by PM3 MO calculations seems that the interaction between the $\alpha$-carbon of the enone and the center carbon of the allene may be possible. However, the intramolecular [2+2] cycloaddition of $\mathbf{2 3 7}$ and $\mathbf{2 4 0}$ is hardly prohibited due to the steric hindrance by the alkyl-substituent at the $\alpha$-position of the enone moiety. For example, overlapping between the MO's of the center carbon of the allene and of the $\alpha$-carbon of these exo-cycloalkenones is blocked.

In the case of the photochemistry of the 1,2-propadienylsubstituted diketone 242a, the intramolecular [2+2] cycloadduct 243a is obtained, because it is considered that some MO interaction between the $\alpha$-carbon and the center carbon of the allene in 242a occurs (Scheme 77). On the other hand, in the case of the photochemistry of the diketone $\mathbf{2 4 2} \mathbf{b}$, the intramolecular [2+2] cycloaddition progresses preferentially.

Successful construction of the tricyclic core of solanoeclepin A (Fig. 8) through intramolecular [2+2] photocycloaddition

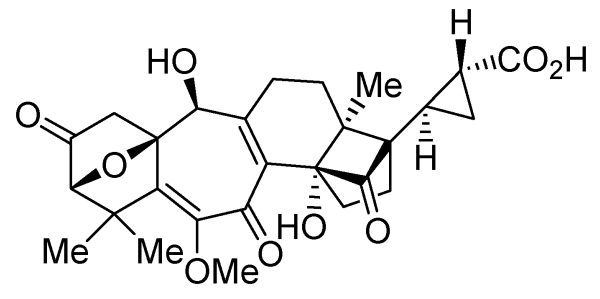

Fig. 8 Structure of solanoeclepin A. 


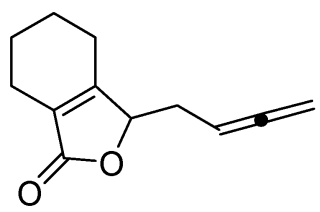

246a

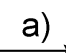

Scheme 78 Examination of the intramolecular [2+2] photocycloaddition of allenene 246a. Reagents and conditions: (a) $h v(300 \mathrm{~nm})$, $\mathrm{MeCN} /$ acetone $(9: 1), 5 \mathrm{~h}$.

of an allene butenolide has been achieved. ${ }^{86} \mathrm{~A}$ model system, compound 246a, was chosen in 2006 by Hiemstra and coworkers to examine the allenene [2+2] photocycloaddition. The key [2+2] cycloaddition of allene 246a which was carried out in a $9: 1$ (acetonitrile/acetone) solution proceeded remarkably well leading to the single product $\mathbf{2 4 7}$ a in good yield (Scheme 78). The exclusive formation of compound 247a emphasizes the great preference for five-membered ring formation in the intramolecular [2+2] cycloaddition. The authors have explained the selectivity by the formation of an allylic radical intermediate $\mathbf{2 4 8}$ instead of the corresponding less stable vinyl radical 249 (Scheme 79). Application of the key photocycloaddition to the appropriate substituted allene butenolides 246b and 246c resulted in the desired crossed cycloadducts $247 \mathrm{~b}$ and $247 \mathrm{c}$ in good yields, which after further elaboration should give the right-hand substructure of the natural product (Scheme 80).

More recently, a similar approach has been described for the stereoselective synthesis of tricyclo[3.3.0]oxohepatene core of bielschowskysin by a [2+2] cycloaddition of an allene butenolide derived of L-malic acid (Scheme 81). ${ }^{87}$ Thus, irradiation of an allene-linked $\gamma$-butenolide $\mathbf{2 5 0}$ cleanly underwent a $[2+2]$ cycloaddition to the single diastereomeric photoadduct 251 in $70 \%$ yield.
249

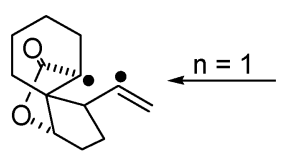

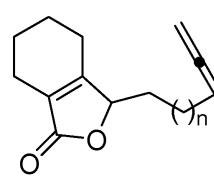

246
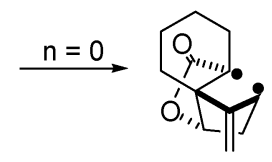

248
Scheme 79 Possible diradical intermediates involved in the intramolecular photocycloaddition of allenenes $\mathbf{2 4 6} .^{86}$

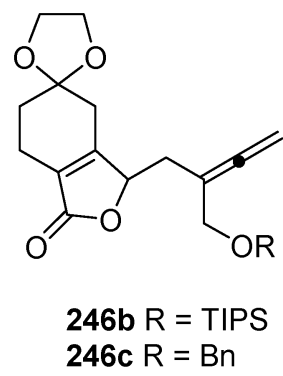

a)

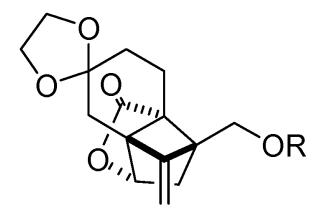

247b $(60 \%)$

247c $(60 \%)$
Scheme 80 Synthesis of the tricyclic core structure of solanoeclepin A. Reagents and conditions: (a) $h v(300 \mathrm{~nm}), \mathrm{MeCN} /$ acetone $(9: 1), 5 \mathrm{~h}$.

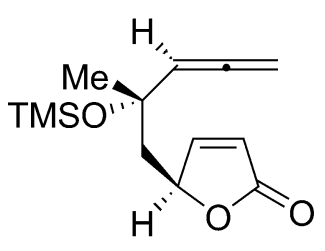

250

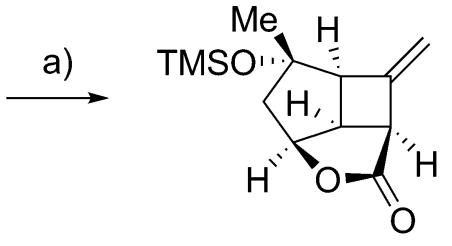

$251(70 \%)$
Scheme 81 Synthesis of the tricyclo[3.3.0]oxoheptane core of bielschowskysin. Reagents and conditions: (a) $h v$, hexane- $\mathrm{CH}_{2} \mathrm{Cl}_{2}$, RT, $12 \mathrm{~h}$.

\section{Metal-catalyzed $[2+2]$ cycloadditions}

Metal catalysis is a very powerful tool for synthetic transformations. In particular, it has provided novel opportunities for the highly selective $[2+2]$ cycloaddition reactions of allenes since complexation of the metal to the allene moiety significantly modifies its reactivity, opening a new route to access to cyclobutane compounds. ${ }^{88}$ In general, this approach has been explained in terms of the reductive elimination of metallacyclopentanes and metallacyclopentenes as the key step in the formation of the four membered rings. In addition to the rate improvements observed in the presence of a metal catalyst, the opportunity to achieve enantioselective transformations by adding chiral ligands is one of the most important characteristics of this strategy.

\subsection{Intermolecular metal-catalyzed $[2+2]$ cycloadditions}

It is noteworthy that metal-catalyzed [2+2] dimerization of allenes takes place with complete control of the regioselectivity and it is independent of the steric and electronic nature of the substituents attached to the allene moiety. Therefore the examples reported so far provide better results, in terms of regioselectivity and yields, than under thermal conditions.

In 2000, Saito and Yamamoto described the regioselective nickel-catalyzed [2+2] annulation of electron-deficient allenes 252 under mild conditions to give the head-to-head cyclodimerization products. The corresponding bismethylenecyclobutanes $\mathbf{2 5 3}$ were isolated as single isomers in good to fair yields (Scheme 82) ${ }^{89}$ However, it is noteworthy that the reaction of 1-hexylallene (1,2-nonadiene) resulted in the formation of a complex mixture which was composed of oligomeric compounds. Therefore, the presence of an electronwithdrawing group is crucial for the [2+2] cycloaddition to proceed efficiently in a highly regioselective manner. In addition, the catalytic reaction did not proceed in the presence of bidentate ligands, assuming that the reaction stopped in the middle of the catalytic cycle of the [2+2] reaction.

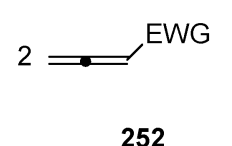

$\mathrm{EWG}=\mathrm{nC}_{6} \mathrm{~F}_{13}, \mathrm{C}_{6} \mathrm{~F}_{5}, \mathrm{CO}_{2} \mathrm{Et}, \mathrm{COEt}$, $\mathrm{COPh}, \mathrm{SO}_{2} \mathrm{Ph}, \mathrm{CON}(\mathrm{Me}) \mathrm{Ph}$
Scheme 82 Nickel(0)-catalyzed [2+2] dimerization of electrondeficient allenes 253. Reagents and conditions: (a) $10 \mathrm{~mol} \% \mathrm{Ni}\left(\mathrm{PPh}_{3}\right)_{4}$, toluene, -15 to $-10{ }^{\circ} \mathrm{C}$ or RT, $0.5-3 \mathrm{~h}$. 

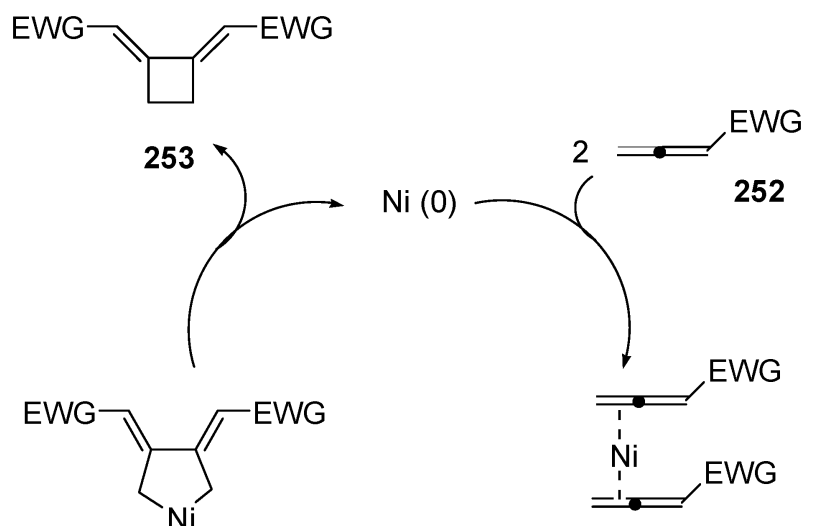

254

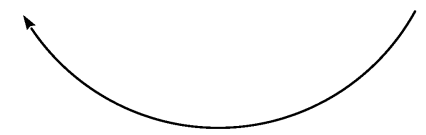

Scheme 83 Proposed mechanism for the nickel(0)-catalyzed [2+2] annulation of electron-deficient allenes $\mathbf{2 5 2}{ }^{89}$

It has been assumed that the reaction proceeds as shown in Scheme 83. The reaction of two molecules of allene 252 under nickel(0) catalysis would produce the nickelacyclopentane $\mathbf{2 5 4}$ by regioselective coordination, followed by $\mathrm{C}-\mathrm{C}$ bond formation. At this step, intermediate $\mathbf{2 5 4}$ would undergo quick reductive elimination to give cyclobutane $\mathbf{2 5 3}$ as the final product. The observed high regioselectivity of the reaction has been explained in terms of the regioselective formation of metallacycle 254, which would be controlled by the stereoelectronic effects of the substituent in allenes $\mathbf{2 5 2}$. The facile reductive elimination of the nickel(0) species from the five-membered metallacycle 254, which is the most characteristic feature of this [2+2] cyclodimerization, would be promoted by the electron-withdrawing groups attached to the allene functionality.

On the other hand, preparation of 1,3-dimethylenecyclobutanes is not straightforward. The current method involves a six-step synthesis. ${ }^{90}$ The simplest access would result from $[2+2]$ allene thermal cyclodimerization. However, it leads to a 1,2-isomer with small amounts of the 1,3 -isomer $(85: 15) .{ }^{91}$ Another interesting synthesis of 1,3-dimethylenecyclobutanes 255 has been described by Dixneuf's group, via a sequential Ru-catalyzed [2+2] cyclodimerization of allenyl boronate $\mathbf{2 5 6}$ and palladium-catalyzed Suzuki coupling (Scheme 84). ${ }^{92}$ The reaction of allenyl boronate $\mathbf{2 5 6}$ in the presence of $5 \mathrm{~mol} \%$ of

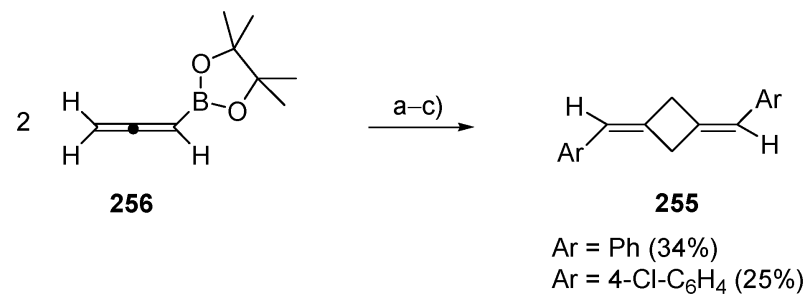

Scheme 84 Synthesis of 1,3-dimethylenecyclobutanes 255 via sequential ruthenium-catalyzed $[2+2]$ cyclodimerization of allenyl boronate and palladium-catalyzed Suzuki coupling. Reagents and conditions: (a) $5 \mathrm{~mol} \%$ [Cp*RuCl(COD)], dioxane, $100{ }^{\circ} \mathrm{C}, 2 \mathrm{~h}$. (b) ArI, $1 \mathrm{~mol} \%$ $\mathrm{Pd}\left(\mathrm{PPh}_{3}\right)_{4}, \mathrm{~K}_{2} \mathrm{CO}_{3}, \mathrm{H}_{2} \mathrm{O}-\mathrm{THF}, 80^{\circ} \mathrm{C}, 2$ h. (c) Recrystallization.
$[\mathrm{Cp} * \mathrm{RuCl}(\mathrm{COD})]$ in refluxing dioxane at $100{ }^{\circ} \mathrm{C}$ followed by addition of an aryl iodide in presence of base $\left(\mathrm{K}_{2} \mathrm{CO}_{3}\right)$ and catalytic amount of $\mathrm{Pd}\left(\mathrm{PPh}_{3}\right)_{4}$, cleanly afforded the [2+2] cyclodimerization products $\mathbf{2 5 5}$. Although a mixture of the two phenyl-substituted isomers $(E, E)$ and $(Z, Z)$ was obtained, fortunately after recrystallization, major isomers $(E, E)$ were isolated in moderate yields.

Total regioselectivity has been achieved during the dimerization of phenylallene 257 by using a sterically hindered ruthenium catalyst, $\left[\mathrm{Cp} * \mathrm{Ru}\left(\mathrm{PPh}_{3}\right)(\mathrm{MeCN})_{2}\right]\left[\mathrm{PF}_{6}\right]$, giving the tail to tail coupling compound 258, in $44 \%$ yield (Scheme 85). ${ }^{92}$

In 2003, Akiyama and co-workers studied the enantioselective [2+2] cycloaddition reaction of 1-methoxyallenylsilanes 259 with an $\alpha$-imino ester catalyzed by the system $\left[\mathrm{Cu}(\mathrm{MeCN})_{4}\right] \mathrm{BF}_{4} /(R)$-Tol-BINAP, affording 3-methyleneazetidine-2-carboxylates 260 in good yields $(60-90 \%)$ and with excellent enantiomeric excesses (58-97\%). ${ }^{93}$ Besides, the acid-catalyzed ring opening of the azetidines $\mathbf{2 6 0}$ has opened a new route to access homochiral acylsilanes $\mathbf{2 6 1}$ quantitatively (Scheme 86).

\subsection{Intramolecular metal-catalyzed $[2+2]$ cycloadditions}

Gold is an effective hetereogeneous and homogeneous catalyst in particular in redox reactions and nucleophilic additions to $\pi$ systems. In fact, although it was considered to be catalytically inactive, gold has shown a rich coordination and organometallic chemistry. ${ }^{94}$ Very recently, Toste and co-workers have developed the first transition-metal catalyzed cycloisomerization of 1,6-allenenes 262 and 263 to alkylidene-cyclobutanes 264 and 265, respectively (Scheme 87). Thus, the reaction of 1,6allenenes 262 and 263 with $5 \mathrm{~mol} \%$ of $\mathrm{Ph}_{3} \mathrm{PAuCl} / \mathrm{AgBF}_{4}$ in $0.1 \mathrm{M} \mathrm{CH} \mathrm{CH}_{2} \mathrm{Cl}_{2}$ afforded the corresponding $[2+2]$ proximal adducts 264 and 265 in excellent yields. A variety
2<smiles>C=Cc1ccccc1</smiles>

257
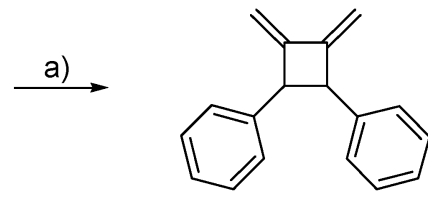

$258(44 \%)$
Scheme 85 Cyclodimerization of phenylallene 257 using a sterically hindered ruthenium catalyst. Reagents and conditions: (a) $10 \mathrm{~mol} \%$ $\left[\mathrm{Cp} * \mathrm{Ru}\left(\mathrm{PPh}_{3}\right)(\mathrm{MeCN})_{2}\right]\left[\mathrm{PF}_{6}\right], \mathrm{DMF}, 110{ }^{\circ} \mathrm{C}, 6 \mathrm{~h}$.

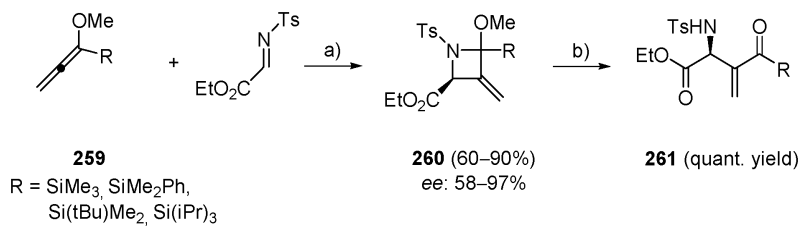

Scheme 86 Copper(I)-catalyzed enantioselective [2+2] cycloaddition of 1-methoxyallenylsilanes $\mathbf{2 5 9}$ with an $\alpha$-imino ester. Reagents and conditions: (a) $10 \mathrm{~mol} \%\left[\mathrm{Cu}(\mathrm{MeCN})_{4}\right] \mathrm{BF}_{4}, 10 \mathrm{~mol} \%$ ( $R$ )-Tol-BINAP, THF, MS $4 \AA,-78{ }^{\circ} \mathrm{C}, 9-24$ h. (b) $1 \mathrm{~N} \mathrm{HCl}, 0{ }^{\circ} \mathrm{C}$. 
<smiles>[X]C/C=C(\[R])Pc1ccccc1</smiles>

262

$\stackrel{\text { a) }}{\longrightarrow}$

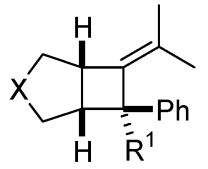

$$
\begin{aligned}
\mathrm{X}= & \mathrm{NTs}, \mathrm{CH}_{2}, \mathrm{C}\left(\mathrm{CO}_{2} \text { allyl }\right)_{2}, \mathrm{C}\left(\mathrm{CO}_{2} \mathrm{Bn}\right)_{2} \\
& \mathrm{C}\left(\mathrm{SO}_{2} \mathrm{Ph}\right)_{2}, \mathrm{C}\left(\mathrm{CO}_{2} \mathrm{Me}\right)_{2}
\end{aligned}
$$$$
\mathrm{R}^{1}=\mathrm{H}, \mathrm{Me}
$$
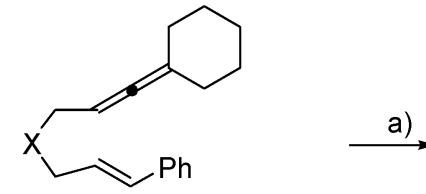

263

$$
\mathrm{X}=\mathrm{C}\left(\mathrm{CO}_{2} \mathrm{Me}\right)_{2}, \mathrm{C}\left(\mathrm{CH}_{2} \mathrm{OMe}\right)_{2}
$$

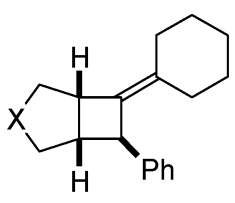

$265(80-83 \%)$

Scheme 87 Gold(I)-catalyzed intramolecular [2+2] cycloaddition of allenenes 262 and 263. Reagents and conditions: (a) $5 \mathrm{~mol} \%$ $\mathrm{Ph}_{3} \mathrm{PAuCl}, 5 \mathrm{~mol} \% \mathrm{AgBF}_{4}, \mathrm{CH}_{2} \mathrm{Cl}_{2}$, RT, 2-24 h.

of aryl-substituted alkenes underwent the gold(I)-catalyzed intramolecular $[2+2]$ cycloaddition with allenes. ${ }^{95}$

This methodology has been successfully applied to the enantioselective version by using a chiral dinuclear gold(I)biarylphosphine complex 266. The reaction of allenenes $\mathbf{2 6 2}$ with the catalyst generated in situ from $3 \mathrm{~mol} \%$ of $(R)$-DTBMSEGPHOS $(\mathrm{AuCl})_{2}$ and $6 \mathrm{~mol} \%$ of $\mathrm{AgBF}_{4}$ afforded the corresponding cyclobutanes $\mathbf{2 6 4}$ in excellent yields and enantioselectivities (Scheme 88).

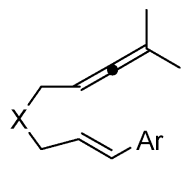

262

$\mathrm{X}=\mathrm{NTs}, \mathrm{C}\left(\mathrm{CO}_{2} \text { allyl }\right)_{2}, \mathrm{C}\left(\mathrm{CO}_{2} \mathrm{Me}\right)_{2}$ $\mathrm{Ar}=\mathrm{Ph}, 4-\mathrm{Cl}_{-} \mathrm{C}_{6} \mathrm{H}_{4}$ 2-Naphthyl, 2-Tolyl

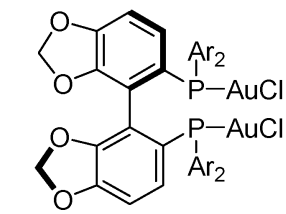

$\mathrm{Ar}=3,5-\mathrm{BBu}_{2}-4-\mathrm{MeO}-\mathrm{C}_{6} \mathrm{H}_{2}$

266

Scheme 88 Enantioselective gold(I)-catalyzed intramolecular allenene [2+2] cycloaddition. Reagents and conditions: (a) $3 \mathrm{~mol} \% \mathbf{2 6 6}, 6 \mathrm{~mol} \%$ $\mathrm{AgBF}_{4}, \mathrm{DCM}(0.1 \mathrm{M}), 4{ }^{\circ} \mathrm{C}$ or RT.

$e e=54-97 \%$
$264(57-92 \%)$

It has been suggested that the gold(I)-catalyzed cycloaddition proceeds through a stepwise mechanism involving a series of carbocationic intermediates. In accordance with a stepwise mechanism involving a benzylic cation, cyclobutanes 264 would be formed from the gold-catalyzed cycloaddition of trans- and cis-alkenes 262. Scheme 89 shows the likely mechanism for the gold(I)-catalyzed reaction. Addition of the nucleophilic alkene to gold(I)-activated allene $\mathbf{2 6 7}$ results in the formation of a carbocationic intermediate 268 . In the presence of methanol, kinetically formed carbocation $\mathbf{2 6 8}$ should be trapped to give trans-cyclopentane 269. The cyclopentane stereochemistry of the methanol adduct would be the opposite to the cyclobutane product, implying that the initial cyclization is reversible. Therefore, in the absence of an

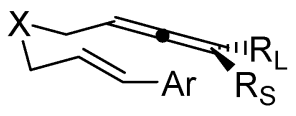

262

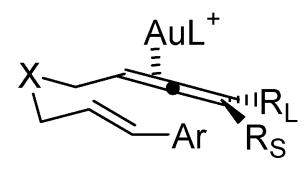

267
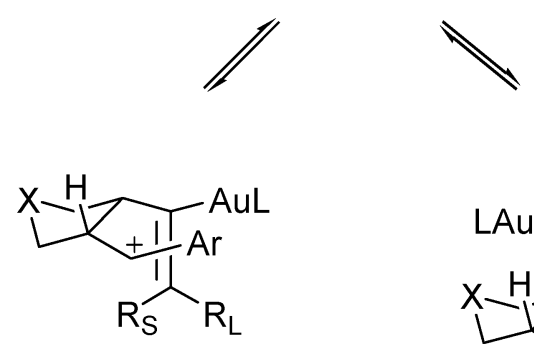

270

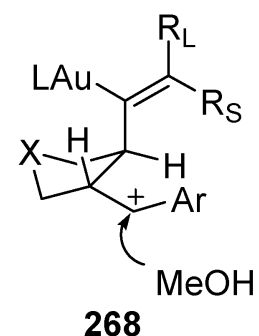<smiles>CC(C)(C)[Mg]</smiles>

264

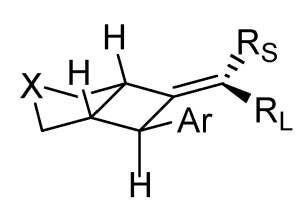

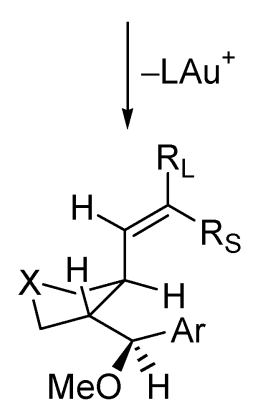

269
Scheme 89 Proposed mechanism of the gold(I)-catalyzed intramolecular $[2+2]$ cycloaddition of allenenes $\mathbf{2 6 2} .^{95}$ 

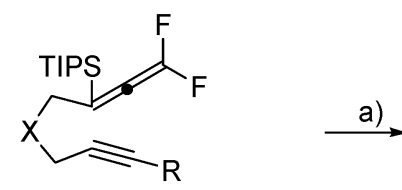

271<smiles>[X]C1=C2CC(S[AsH2])=C2C(F)(F)C1[R]</smiles>

$272(72-92 \%)$ $\mathrm{X}=\mathrm{C}\left(\mathrm{CO}_{2} \mathrm{Me}\right)_{2}, \mathrm{NTs} ; \mathrm{R}=\mathrm{H}, \mathrm{Me}, \mathrm{nPr}, \mathrm{Ph}$

Scheme 90 Mo-catalyzed intramolecular $[2+2]$ cycloaddition of gem-difluoroallenynes 271. Reagents and conditions: (a) $10 \mathrm{~mol} \%$ $\mathrm{Mo}(\mathrm{CO})_{6}, \mathrm{DMSO} /$ toluene, $100{ }^{\circ} \mathrm{C}$.

exogenous nucleophile, the reaction proceeds through cis-disubstituted intermediates 270. Cyclobutanes 264 are then formed from the reaction of vinyl-gold with benzylic carbocations.

In addition to the excellent yields and $e e$ 's, this methodology has turned out to be very elegant because proximal adducts are obtained as the only isomers. However, under thermal conditions, distal adducts are regioselectively obtained for related allenyl systems (see compounds 151, Scheme 47 and 48).

During studies of the Pauson-Khand reaction of functionalized gem-difluoroallenes 271, Hammond's group has discovered that under molybdenum-catalyzed conditions the intramolecular $[2+2+1]$ cycloaddition did not occur, but novel fused gem-difluorocyclobutenes $\mathbf{2 7 2}$ were isolated instead (Scheme 90). ${ }^{96}$ Mechanistically, this reaction has been explained as a formal [2+2] cycloaddition, with the molybdacycle undergoing a reductive elimination, rather than a $\mathrm{CO}$ insertion.

In the same context, Cook and co-workers have observed the formation of the hybrid [2+2]/[2+2+1] cycloadducts $\mathbf{2 7 3}$ and 274 during the molybdenum-mediated reaction of the bisallene-bisalkyne 201. ${ }^{97}$ Thus, when compound 201 was heated in toluene at $100{ }^{\circ} \mathrm{C}$ in the presence of a slight excess of $\mathrm{Mo}(\mathrm{CO})_{6}$ (2.2 equiv.) and 10 equiv. of DMSO, a mixture of diastereomers composed of the [5.5.5.4] tetracycles 273 and 274 in a ratio of 5:4 was obtained (Scheme 91). Interestingly, the Mo-catalyzed reaction of compound 201 promotes the $[2+2]$ cycloaddition of both allenynes which was not the case for the thermal conditions described in Scheme 62 .

More recently, Mukai has described the same competitive reaction in bisallenes 275 (Scheme 92). ${ }^{98}$ Thus, in the rhodium(I)catalyzed reaction of bisallenes $\mathbf{2 7 5}$, bicyclo[5.3.0] compounds $\mathbf{2 7 6}$ have been obtained together with [2+2] cycloadducts 277.

$\mathrm{Oh}$ and co-workers have studied the palladium-catalyzed $[2+2]$ cycloaddition of allenynes $\mathbf{1 7 8}$ and $\mathbf{1 7 9}$, tested previously
201

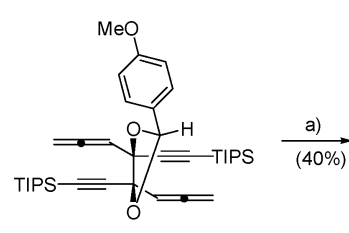

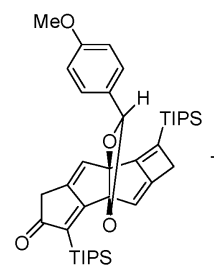

273

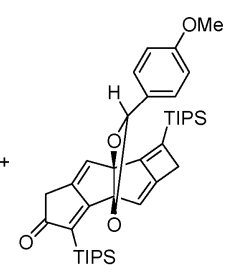

274
Scheme 91 Molybdenum-mediated $[2+2] /[2+2+1]$ cycloaddition of the bisallene-bisalkyne 201. Reagents and conditions: (a) 2.2 equiv. $\mathrm{Mo}(\mathrm{CO})_{6}, 10$ equiv. DMSO, toluene, $100{ }^{\circ} \mathrm{C}$.
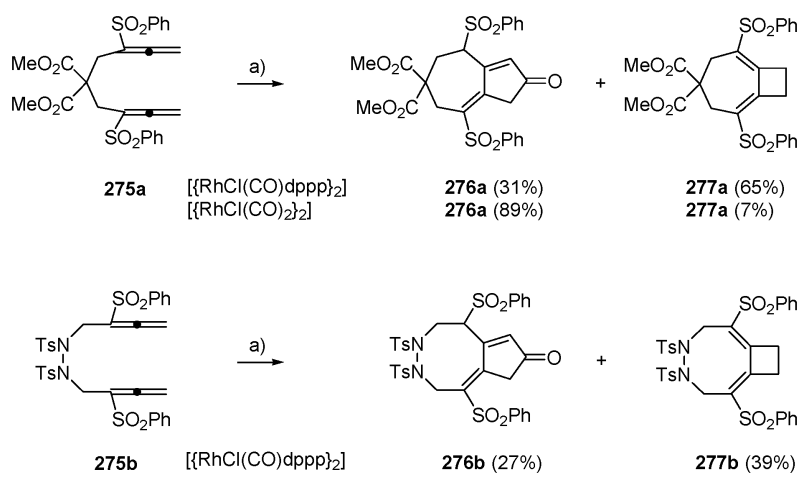

Scheme $92 \mathrm{Rh}(\mathrm{I})$-catalyzed intramolecular [2+2] cycloaddition of bisallenes 275. Reagents and conditions: (a) $5 \mathrm{~mol} \% \mathrm{Rh}$ (I), toluene, $80{ }^{\circ} \mathrm{C}, \mathrm{CO}$ (1 atm.), $1-18 \mathrm{~h}$.

under microwave conditions, resulting in the formation of the corresponding distal cycloadducts $\mathbf{1 8 0}$ and $\mathbf{1 8 1}$ in good yields (Scheme 93). ${ }^{54}$ The optimal conditions for this reaction were found to be the use of $\mathrm{Pd}\left(\mathrm{PPh}_{3}\right)_{2} \mathrm{Cl}_{2}(5 \mathrm{~mol} \%)$ in refluxing toluene, obtaining the corresponding bicyclic compounds without forming any other side product. However, allenynes 179d and 179e were inert under the metal-catalyzed conditions, showing the microwave irradiation to be a more efficient technique for this family of allenynes (see Scheme 55).

From a mechanistic point of view, it is believed that the palladium(II) first forms a $\pi$-complex with both the triple bond and a double bond of substrates 178 and 179. Such $\pi$-complexes are known to undergo migratory $\mathrm{C}-\mathrm{C}$ coupling to give palladacyclopentenes 278. Reductive elimination of intermediates 278 would produce compounds 180 and 181 and would regenerate palladium(II) for the next cycle (Scheme 94).

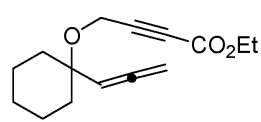

178

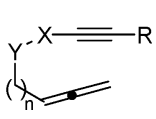

179b $\mathrm{n}=0, \mathrm{X}=\mathrm{CH}_{2} \mathrm{OH}, \mathrm{Y}=\mathrm{C}(\mathrm{Me})_{2}, \mathrm{R}=\mathrm{CO}_{2} \mathrm{Et}$

179c $\mathrm{n}=0, \mathrm{X}=\mathrm{CH}_{2} \mathrm{OH}, \mathrm{Y}=\mathrm{C}(\mathrm{Me})_{2}, \mathrm{R}=\mathrm{Ph}$

179d $n=2, X=\mathrm{CH}_{2}, Y=\mathrm{C}\left(\mathrm{CO}_{2} \mathrm{Et}\right)_{2}, \mathrm{R}=\mathrm{CO}_{2} \mathrm{Et}$

179e $\mathrm{n}=2, \mathrm{X}=\mathrm{CH}_{2}, \mathrm{Y}=\mathrm{pTs}, \mathrm{R}=\mathrm{CO}_{2} \mathrm{Et}$

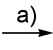

$\stackrel{\text { a) }}{\longrightarrow}$
Scheme 93 Pd-catalyzed intramolecular [2+2] cycloaddition of allenynes $\mathbf{1 7 8}$ and 181. Reagents and conditions: (a) $5 \mathrm{~mol} \%$ $\mathrm{PdCl}_{2}\left(\mathrm{PPh}_{3}\right)_{2}$, toluene, $110{ }^{\circ} \mathrm{C}, 12 \mathrm{~h}$.

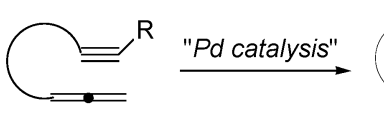

178,179

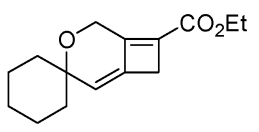

$180(81 \%)$<smiles>[Y][Y]1cc2c(c1)C([R])=C2</smiles>

$181 \mathrm{~b}(87 \%)$

181c $(61 \%)$

$181 \mathrm{~d}(0 \%)$

181e $(0 \%)$
Scheme 94 Proposed mechanism for the Pd-catalyzed [2+2] cycloaddition of allenynes $\mathbf{1 7 8}$ and $\mathbf{1 7 9} .^{54}$ 


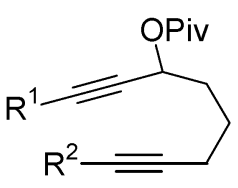

279 $\stackrel{\text { a) }}{\longrightarrow}$<smiles>[R]C(=O)C1=C([R])C2CCCC12</smiles>

$281(72-80 \%)$
$\mathrm{R}^{1}=\mathrm{nBu}$, cyclohexyl, $\mathrm{Ph}$

$\mathrm{R}^{2}=1-\mathrm{Np}, \mathrm{Ph}$

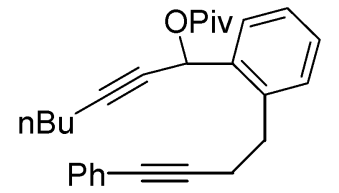

280

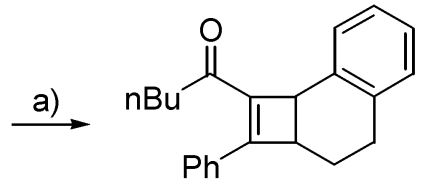

$282(75 \%)$
Scheme 95 Gold-catalyzed [2+2] cycloaddition of masked allenynes, alkadiynylic pivaloates $\mathbf{2 7 9}$ and $\mathbf{2 8 0}$. Reagents and conditions: (a) $10 \mathrm{~mol} \% \mathrm{AuCl}\left(\mathrm{PPh}_{3}\right), 10 \mathrm{~mol}^{\%} \mathrm{AgSbF}_{6}, \mathrm{DCE}, 25-80{ }^{\circ} \mathrm{C}, 1-15 \mathrm{~h}$.

More recently, the same authors have reported the synthesis of fused cyclobutenes via gold-catalyzed [2+2] cyclization of 1,8-alkadiynylic pivaloates, masked allenynes. ${ }^{99}$ Substrates $\mathbf{2 7 9}$ and $\mathbf{2 8 0}$ were smoothly transformed to the [3.2.0] and [4.2.0] bicycles $\mathbf{2 8 1}$ and $\mathbf{2 8 2}$ by treatment with a catalytic amount of $\mathrm{AuCl}\left(\mathrm{PPh}_{3}\right)$ (Scheme 95). The corresponding cycloadducts were obtained in good yields in all cases.

A plausible mechanism for the gold-catalyzed cyclization of bisalkynyl pivaloates $\mathbf{2 7 9}$ and $\mathbf{2 8 0}$ has been proposed (Scheme 96). Firstly, coordination of gold(I) to the propargylic ester would induce migration of the pivaloate to give the allene intermediate 283. The allene $\mathbf{2 8 3}$ underwent $[2+2]$ cycloaddition to give the intermediate $\mathbf{2 8 4}$, which led to the isolated products $\mathbf{2 8 1}$ and $\mathbf{2 8 2}$ upon hydrolysis.

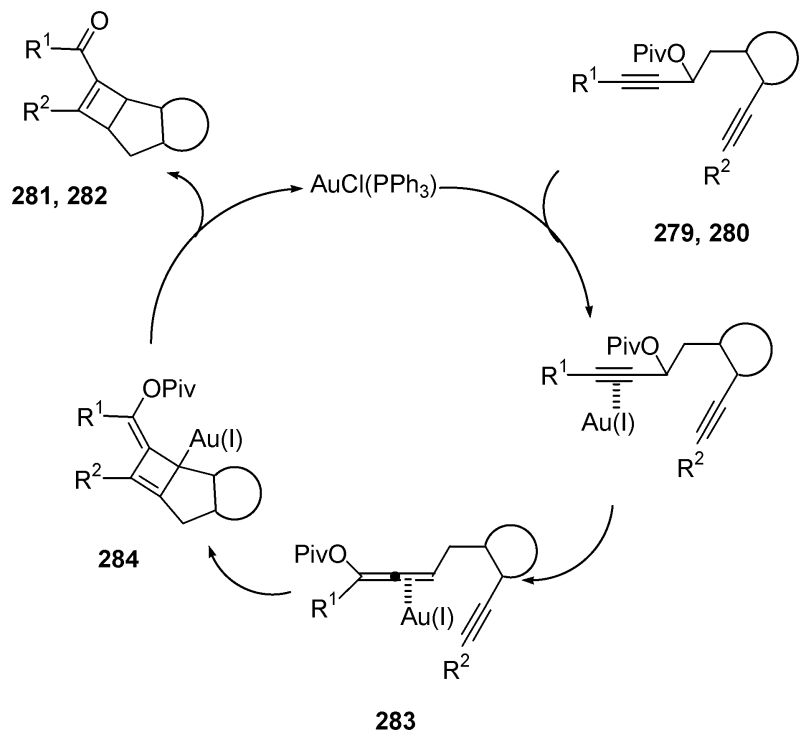

Scheme 96 Proposed mechanism for the gold-catalyzed [2+2] cycloaddition of masked allenynes, alkadiynylic pivaloates 279 and 280. ${ }^{99}$

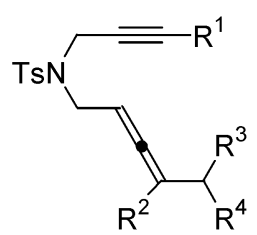

285

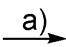<smiles>[R]C([R])=C([R])C1=C([R])C2CN([As])CC12</smiles>

$286(35-85 \%)$

$$
\begin{aligned}
\mathrm{R}^{1}= & 3-\mathrm{MeO}-\mathrm{C}_{6} \mathrm{H}_{4}, 4-\mathrm{MeO}-\mathrm{C}_{6} \mathrm{H}_{4}, \\
& \text { 2-Np, } \mathrm{Ph}, \mathrm{Me} \\
\mathrm{R}^{2}= & \mathrm{Me}, \mathrm{iPr} ; \mathrm{R}^{3}=\mathrm{H}, \mathrm{Me}
\end{aligned}
$$

Scheme 97 Cycloisomerization of allenynes 285 catalyzed by $\mathrm{PtCl}_{2}$. Reagents and conditions: (a) $10 \mathrm{~mol} \% \mathrm{PtCl}_{2}$, toluene, $80{ }^{\circ} \mathrm{C}, 12-24 \mathrm{~h}$.

Platinum complexes have also been used in cycloisomerization reactions of enynes, which afford a wide spectrum of products depending on structural variations such as the nature of the substituents on the enyne and the tethers connecting these groups. ${ }^{100}$ Murakami and co-workers have reported that the treatment of compounds $\mathbf{2 8 5}$ with a catalytic amount of platinum(II) chloride in toluene at $80{ }^{\circ} \mathrm{C}$ cleanly afforded the corresponding bicyclic cyclobutenes $\mathbf{2 8 6}$ in good yields (Scheme 97). ${ }^{101}$

Scheme 98 illustrates the mechanism for the formation of cyclobutenes 286. Coordination of the alkyne moiety of $\mathbf{2 8 5}$ to $\mathrm{PtCl}_{2}$ would form a platinum(II)-alkyne complex 287, which will undergo endo-cyclization with the proximal allenic carbon-carbon double bond to form a seven membered ring. The resulting carbocation $\mathbf{2 8 8}$ is in resonance with $\mathbf{2 8 9}, \mathbf{2 9 0}$ and 291. The relatively more stable carbocation 289 would abstract a proton from the methyl group, and finally, cyclobutenylplatinum hydride $\mathbf{2 9 2}$ would undergo reductive elimination to provide 286 .

The chemistry of bis-allenes is an emerging area and recently several reports have been published under metal-catalyzed conditions. ${ }^{102}$ Thus, in 2006, Ma's group has reported the $[2+2]$ cycloaddition in 1,5-bisallenes 293-295 using palladium( $(0)$, as metal promoter, in the presence of $n \mathrm{Bu}_{4} \mathrm{NI}$ and $\mathrm{K}_{2} \mathrm{CO}_{3}$,

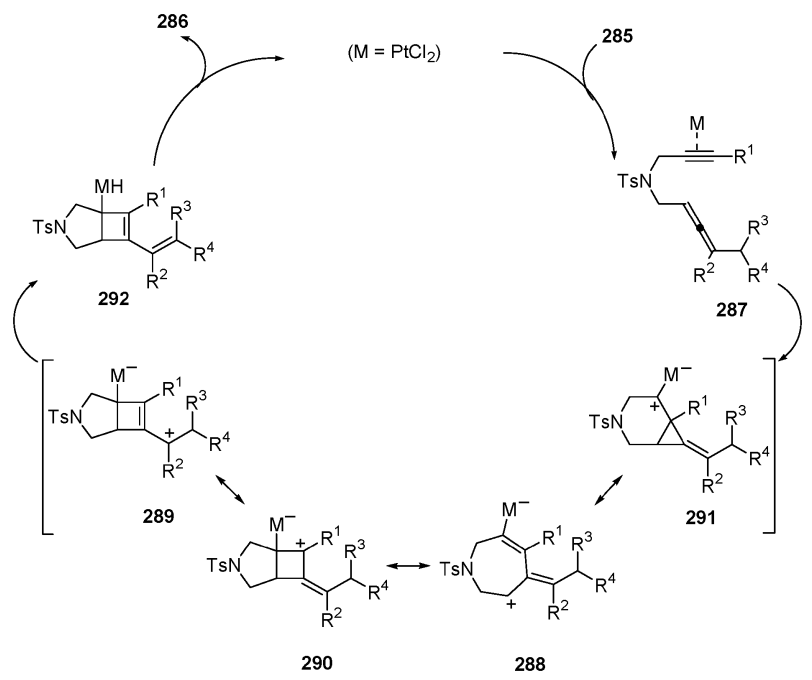

Scheme 98 Mechanism for the cycloisomerization of allenynes $\mathbf{2 8 5}$ catalyzed by $\mathrm{PtCl}_{2}{ }^{101}$ 
293

$\mathrm{X}=\mathrm{NTs}, \mathrm{C}\left(\mathrm{SO}_{2} \mathrm{Ph}\right)_{2}, \mathrm{C}\left(\mathrm{CO}_{2} \mathrm{Et}\right)_{2}$

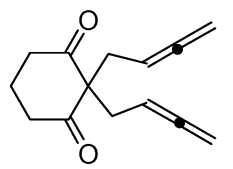

294

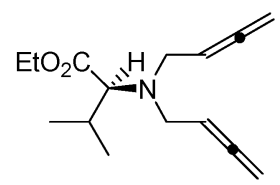

(S)-295
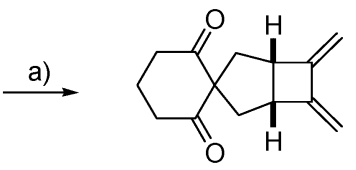

297 (54\%)

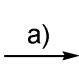

298 (66\%, ee: $>99 \%)$

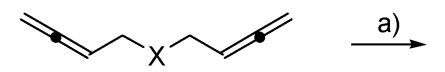

Scheme $99 \operatorname{Pd}(0)$-catalyzed [2+2] cycloaddition of 1,5-bisallenes 293-295. Reagents and conditions: (a) $10 \mathrm{~mol} \% \quad\left[\mathrm{Pd}\left(\mathrm{PPh}_{3}\right)_{4}\right]$, $\mathrm{K}_{2} \mathrm{CO}_{3}, n \mathrm{Bu}_{4} \mathrm{NI}, 80-85^{\circ} \mathrm{C}, 4-19 \mathrm{~h}$.

affording bicycle [3.2.0] products 296-298 in moderate to good yields $(54-66 \%)$ without formation of other regioisomers (Scheme 99). ${ }^{103}$ Interestingly, reaction of compound $(S)$-295 containing the chiral L-valine ester moiety, afforded bicycle 298 in greater than $99 \% e e$, which indicates that the reaction proceeded without racemization of the $\alpha$-amino acid ester. However, compounds with substituents on the proximal side did not provide the expected [3.2.0] products, probably due to steric reasons. In the course of optimizing the reaction, it was noted that the reaction failed on using $n \mathrm{Bu}_{4} \mathrm{NI}$ alone. The $\mathrm{I}^{-}$ species in $n \mathrm{Bu}_{4} \mathrm{NI}$ may act as a ligand facilitating the reductive elimination process.

A plausible model for the highly diastereoselective $\operatorname{Pd}(0)$-catalyzed [2+2] cycloaddition of 1,5-bisallenyl compound (S)-295 is shown in Scheme 100. Coordination between the Pd atom and the lone pair of electrons of the $\mathrm{N}$ atom in the intermediate 299, along with the requirement of the $\mathrm{Pd}$ atom to be distant from the bulkier $\mathrm{CO}_{2}$ Et group, would lead to the exclusive formation of 298. The inversion of the nitrogen center may be very difficult in this case because of the presence of the bicyclic skeleton.

Although Ma's contribution is an isolated example, this methodology opens a new route to study the intra- and intermolecular [2+2] dimerization of bisallenes in a regioand stereocontrolled manner under mild conditions.

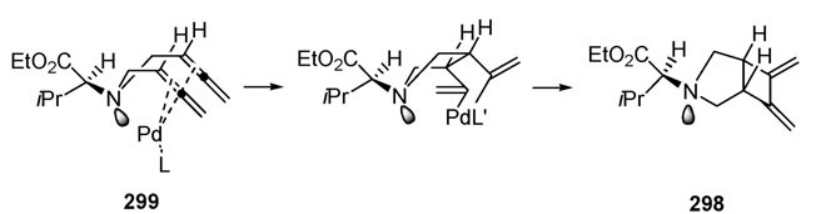

Scheme 100 Model accounting for the diastereoselective palladiumcatalyzed $[2+2]$ cycloaddition of 1,5 -bisallene $(S)-\mathbf{2 9 7} .^{103}$

\section{Concluding remarks}

In this review we have presented the most recent advances in $[2+2]$ cycloaddition of allenes, showing this methodology to be an established process to access a large number of polycyclic structures containing the cyclobutane skeleton. The reactions discussed herein demonstrate the high synthetic potential of allenes undergoing [2+2] cycloaddition under several reaction conditions with almost complete regioselectivity, which is controlled by the stereo-electronic nature of the substituents attached to the allene moiety. In addition it has also been demonstrated that metal catalyzed [2+2] cycloaddition proceed in most cases with better yields and controlled regioselectivity, which is not accessible under thermal or photochemical conditions. It is believed that the continued and renewed investigation on [2+2] cycloadditions of allenes will enrich the synthetic applications to access a high diversity of complex structures, including natural products.

\section{Abbreviations}

Ac

$\mathrm{AO}$

BINAP

$\mathrm{Bn}$

$\mathrm{Bz}$

$\mathrm{Cp}$

COD

DCE

DEF

DEM

DMA

DME

DMF

dr

DTBM-SEGPHOS

dppp

ee

EWG

HP

KIE

LiHMDS

MO

MOM

MS

Mts

MWI

NPM

$\mathrm{Np}$

Piv

PMO

PMP

$p$-TSOH

py

TIPS

TMS acetyl

atomic orbital

2,2'-bis(diphenylphosphino)-1,1'binaphthyl

benzyl

benzoyl

cyclopentenyl

cyclooctadienyl

1,1-dichloroethane

diethyl fumarate

diethyl maleate

dimethylallene

1,2-dimethoxyethane

dimethylformamide

diastereomeric ratio

(4,4' -bi-1,3-benzodioxole)-5,5'-diyl)-

bis[bis(3,5-di-tert-butyl-4-methoxy-

phenyl)phosphine]

1,3-bis(diphenylphosphino)propane

enantiomeric excess

electron withdrawing group

high pressure

kinetic isotope effect

lithium hexamethyldisilazide

molecular orbital theory

methoxymethyl

molecular sieves

2,4,6-trimethylphenylsulfonyl

microwave irradiation

$N$-phenylmaleimide

naphthyl

pivaloyl

perturbation molecular orbital theory

p-methoxyphenyl

$p$-toluenesulfonic acid

pyridine

triisopropylsilyl

trimethylsilyl 
Tol

Ts

tolyl

$p$-toluenesulfonyl

\section{Acknowledgements}

Support for this work by the DGI-MEC (Project CTQ200610292), UCM-BSCH and Universidad Complutense de Madrid (Grant GR58/08) are gratefully acknowledged. C. A. thanks the MEC for a Ramón y Cajal Contract co-financed by the European Social Fund.

\section{References}

1 The Chemistry of Allenes, ed. S. R. Landor, Academic Press, London, 1982, vol. 1, Modern Allene Chemistry, ed. N. Krause and A. S. K. Hashmi, Wiley-VCH, Weinheim, 2004, vol. 1 and 2, and references therein; Cumulenes and Allenes, Science of Synthesis, Houben-Weyl Method of Molecular Transformation, ed. N. Krause, George Tieme, Sttutgart, 2007,vol. 44.

2 D. J. Pasto, Tetrahedron, 1984, 40, 2805-2827; R. Zimmer, C. U. Dinesh, E. Nandanan and F. A. Khan, Chem. Rev., 2000, 100, 3067-3125; H.-U. Reissig, W. Schade, G. M. O. Amombo, R. Pulz and A. Hausherr, Pure Appl. Chem., 2002, 74, 175-180; A. Hoffmann-Röder and N. Krause, Angew. Chem., Int. Ed., 2004, 43, 1196-1216; S. Ma, Chem. Rev., 2005, 105, 2829-2872; $\mathrm{S}$. Ma, in Topics in Organometallic Chemistry, ed. J. Tsuji, SpringerVerlag, Heidelberg, 2005, pp. 183-210; M. Brasholz, H.-U. Reissig and R. Zimmer, Acc. Chem. Res., 2009, 42, 45-56.

3 J. E. Baldwin, in Comprehensive Organic Synthesis, ed. B. M. Trost and I. Fleming, Pergamon Press, Oxford, 1991, vol. 5, pp. 63-84; M. T. Crimmins, in Comprehensive Organic Synthesis, ed. B. M. Trost and I. Fleming, Pergamon Press, Oxford, 1991, vol 5, pp. 123-150; The Chemistry of Cyclobutanes, ed. Z. Rappoport and J. F. Liebman, Wiley, 2005; Stereoselective Synthesis, Methods of Organic Chemistry (Houben-Weyl), ed. G. Helmchen, R. W. Hoffmann, J. Mulzer and E. Schaumann, Thieme: Stuttgart, Germany, 1996, vol. 5, pp. 3061-3125; E. Lee-Ruff and G. Mladenova, Chem. Rev., 2003, 103, 1449-1483.

4 For a recent review, see: J. C. Namyslo and D. Kaufmann, Chem. Rev., 2003, 103, 1485-1538.

5 For selected, recent examples of fragmentation and ring-expansion of substituted cyclobutanes, see: M. Murakami, S. Ashida and T. Matsuda, J. Am. Chem. Soc., 2005, 127, 6932-6933; P. A. Wender, N. M. Deschamps and R. Sun, Angew. Chem., Int. Ed., 2006, 45, 3957-3960; N. Maulide and I. E. Markó, Org. Lett., 2007, 9, 3757-3760; B. M. Trost and J. Xie, J. Am. Chem. Soc., 2008, 130, 6231-6242; J. M. Um, H. Xu, K. N. Houk and W. Tang, J. Am. Chem. Soc., 2009, 131, 6664-6665.

6 R. Hoffmann and R. B. Woodward, J. Am. Chem. Soc., 1965, 87, 2046-2048; R. B. Woodward and R. Hoffmann, Angew. Chem., Int. Ed. Engl., 1969, 8, 781-853; in The Conservation of Orbital Symmetry, ed. R. B. Woodward and R. Hoffmann, Academic Press, New York, 1970.

7 S. Inagaki, H. Fujimoto and K. Fukui, J. Am. Chem. Soc., 1976, 98, 4693-4701.

8 R. S. Varma, Pure Appl. Chem., 2001, 73, 193-198; P. Lidstrom, J. Tierney, B. Wathey and J. Westman, Tetrahedron, 2001, 57, 9225-9283; C. O. Kappe, Angew. Chem., Int. Ed., 2004, 43, 6250-6284; M. Nüchter, B. Ondruschka, W. Bonrath and A. Gum, Green Chem., 2004, 6, 128-141; B. A. Roberts and C. R. Strauss, Acc. Chem. Res., 2005, 38, 653-661; N. Hoffmann, Chem. Rev., 2008, 108, 1052-1103.

9 A. T. Blomquist and J. A. Verdol, J. Am. Chem. Soc., 1956, 78, 109-112; H. A. Staab and H.-A. Kurmeier, Chem. Ber., 1968, 101, 2697-2708; A. Roedig and N. Detzer, Justus Liebigs Ann. Chem., 1971, 749, 153-158; A. Roedig, N. Detzer and G. Bonse, Justus Liebigs Ann. Chem., 1971, 752, 60-63; N. Detzer and A. Roedig, Tetrahedron, 1971, 27, 5697-5703; S. R. Byrn, E. Maverick, O. J. Muscio, Jr., K. N. Trueblood and T. L. Jacobs, J. Am. Chem. Soc., 1971, 93, 6680-6682; P. Binger and U. Schuchardt, Chem. Ber., 1980, 113, 1063-1071; H. Jendralla and K. Laumen, Chem. Ber., 1983, 116, 2136-2164; R. W. Saalfrank, W. Paul and
P. Schierling, Chem. Ber., 1985, 118, 2150-2155; A. J. Brattesani, E. Maverick, O. J. Muscio, Jr. and T. L. Jacobs, J. Org. Chem., 1992, 57, 7346-7349.

10 J. J. Gajewski and C. N. Shih, J. Org. Chem., 1972, 37, 64-68.

11 A. Roedig and N. Defzer, Justus Liebigs Ann. Chem., 1967, 710, 1-6; A. Roedig, Angew. Chem., Int. Ed. Engl., 1969, 8, 150-151; W. R. Moore, R. D. Bach and T. M. Ozretich, J. Am. Chem. Soc., 1969, 91，5918-5919; T. L. Jacobs, J. R. McClenon and O. J. Muscio, Jr., J. Am. Chem. Soc., 1969, 91, 6038-6041; E. V. Dehmlow, Tetrahedron Lett., 1969, 10, 4283-4286; J. J. Gajewski and W. A. Black, Tetrahedron Lett., 1970, 11, 899-902.

12 R. Montaigne and L. Ghosez, Angew. Chem., Int. Ed. Engl., 1968, 7, 221; R. Huisgen and P. Otto, J. Am. Chem. Soc., 1968, 90, 5342-5343; R. Huigen, L. A. Feiler and P. Otto, Tetrahedron Lett., 1968, 9, 4485-4490; W. T. Brady and R. Roe Jr, J. Am. Chem. Soc., 1970, 92, 4618-4621; H. M. Frey and N. S. Issacs, J. Chem. Soc. B, 1970, 830-832; J. E. Baldwin and J. A. Kapecki, J. Am. Chem. Soc., 1970, 92, 4868-4873.

13 W. H. Knoth and D. D. Coffman, J. Am. Chem. Soc., 1960, 82, 3873-3875.

14 C. W. N. Crumper, Z. T. Formum, P. M. Greaves and S. R. Landor, J. Chem. Soc., Perkin Trans. 2, 1973, 885-888.

15 W. R. Dolbier and S. H. Dai, J. Am. Chem. Soc., 1968, 90, 5028-5030; W. R. Dolbier, Jr. and S. H. Dai, J. Am. Chem. Soc., 1970, 92, 1774-1776; S. H. Dai and W. R. Dolbier, Jr., J. Am. Chem. Soc., 1972, 94, 3946-3952.

16 W. R. Moore, R. D. Bach and T. M. Ozretich, J. Am. Chem. Soc., 1969, 91, 5918-5919; J. E. Baldwin and U. V. Roy, J. Chem. Soc. D, 1969, 1225-1126.

17 O. J. Muscio, Jr. and T. L. Jacobs, Tetrahedron Lett., 1969, 10, 2867-2870; T. L. Jacobs, J. R. McClenon and O. J. Muscio, Jr., J. Am. Chem. Soc., 1969, 91, 6038-6041; J. J. Gajewski and C. N. Shih, J. Am. Chem. Soc., 1969, 91, 5900-5901; T. L. Jacobs and O. J. Muscio, Jr., Tetrahedron Lett., 1970, 11, 4829-4832.

18 B. G. Odell, R. Hoffmann and A. Imamura, J. Chem. Soc. B, 1970, 1675-1682.

19 T. J. Levek and E. F. Kiefer, J. Am. Chem. Soc., 1976, 98, $1875-1879$

20 J. J. Gajewski and C. N. Shih, J. Am. Chem. Soc., 1972, 94, $1675-1678$.

21 D. J. Pasto, J. Am. Chem. Soc., 1979, 101, 37-46.

22 E. F. Kiefer and M. Y. Okamura, J. Am. Chem. Soc., 1968, 90, 4187-4189.

23 J. E. Baldwin and U. V. Roy, J. Chem. Soc. D, 1969, 1225-1226.

24 D. J. Pasto and S. E. Warren, J. Am. Chem. Soc., 1982, 104, $3670-3676$

25 D. J. Pasto, S. E. Warren and M. A. Morrison, J. Org. Chem., 1981, 46, 2837-2841; D. J. Pasto and S. E. Warren, J. Org. Chem., 1981, 46, 2842-2846; D. J. Pasto and G. L'Hermine, J. Org. Chem., 1990, 55, 685-694.

26 D. J. Pasto, P. F. Heid and S. E. Warren, J. Am. Chem. Soc., 1982, 104, 3676-3685.

27 D. J. Pasto and S.-H. Yang, J. Am. Chem. Soc., 1984, 106, $152-157$.

28 D. J. Pasto and K. D. Sugi, J. Org. Chem., 1991, 56, 3795-3801.

29 D. J. Pasto and K. D. Sugi, J. Org. Chem., 1991, 56, 6216-6224.

30 D. J. Pasto and K. D. Sugi, J. Org. Chem., 1992, 57, 12-17.

31 D. J. Pasto and W. Kong, J. Org. Chem., 1988, 53, 4807-4810.

32 D. J. Pasto and K. D. Sugi, J. Org. Chem., 1992, 57, 1146-1150.

33 Y. Gu, T. Hama and G. B. Hammond, Chem. Commun., 2000, 395-396.

34 L. E. Harrington, J. F. Britten and M. J. McGlinchey, Org. Lett., 2004, 6, 787-790.

35 E. V. Banide, Y. Ortin, C. M. Seward, L. E. Harrington, H. Müller-Bunz and M. J. McGlinchey, Chem.-Eur. J., 2006, 12, 3275-3286.

36 E. V. Banide, B. C. Molloy, Y. Ortin, H. Müller-Bunz and M. J. McGlinchey, Eur. J. Org. Chem., 2007, 2611-2622.

37 N. N. B. Kumar, M. Chakravarty, N. S. Kumar, K. V. Sajna and K. C. K. Swamy, J. Chem. Sci., 2009, 121, 23-36.

38 M. E. Jung and N. Nishimura, J. Am. Chem. Soc., 1999, 121, 3529-3530; M. E. Jung, N. Nishimura and A. R. Novack, 
J. Am. Chem. Soc., 2005, 127, 11206-11207; M. E. Jung and A. R. Novack, Tetrahedron Lett., 2005, 46, 8237-8240.

39 M. E. Jung and N. Nishimura, Org. Lett., 2001, 3, 2113-2115.

40 M. E. Jung and M. Murakami, Org. Lett., 2006, 8, 5857-5859.

41 M. E. Jung and M. Murakami, Org. Lett., 2007, 9, 461-463.

42 M. Kimura, Y. Horino, Y. Wakamiya, T. Okajima and Y. Tamaru, J. Am. Chem. Soc., 1997, 119, 10869-10870; Y. Horino, M. Kimura, S. Tanaka, T. Okajima and Y. Tamaru, Chem.-Eur. J., 2003, 9, 2419-2438.

43 V. Nair, D. Sethumadhavan, S. M. Nair, P. Shanmugam, P. M. Treesa and G. K. Eigendorf, Synthesis, 2002, 1655-1657.

44 R. W. M. Aben, S. Braverman and H. W. Scheeren, Eur. J. Org. Chem., 2003, 894-897.

45 M. Ogasawara, A. Okada, K. Nakajima and T. Takahashi, Org. Lett., 2009, 11, 177-180.

46 A. Padwa, M. A. Filipkowski, M. Meske, S. H. Watterson and Z. Ni, J. Am. Chem. Soc., 1993, 115, 3776-3777; A. Padwa, M. Meske, S. S. Murphree, S. H. Watterson and Z. Ni, J. Am. Chem. Soc., 1995, 117, 7071-7080.

47 A. Padwa, H. Lipka, S. H. Watterson and S. S. Murphree, J. Org. Chem., 2003, 68, 6238-6250.

48 T. V. Hansen, L. Skattebøl and Y. Strenstrøm, Tetrahedron, 2003, 59, 3461-3466.

49 B. Alcaide, P. Almendros, C. Aragoncillo, M. C. Redondo and M. R. Torres, Chem.-Eur. J., 2006, 12, 1539-1546.

50 H. Ohno, T. Mizutani, Y. Kadoh, A. Aso, K. Miyamura, N. Fujii and T. Tanaka, J. Org. Chem., 2007, 72, 4378-4389.

51 C. Mukai, Y. Hara, Y. Miyashita and F. Inagaki, J. Org. Chem., 2007, 72, 4454-4461.

52 X. Jiang and S. Ma, Tetrahedron, 2007, 63, 7589-7595.

53 K. M. Brummond and D. Chen, Org. Lett., 2005, 7, 3473-3475.

54 C. H. Oh, D. I. Park, S. H. Jung, V. R. Reddy, A. K. Gupta and Y. M. Kim, Synlett, 2005, 2092-2094; C. H. Oh, A. K. Gupta, D. I. Park and N. Kim, Chem. Commun., 2005, 5670-5672.

55 T. V. Ovaska and R. E. Kyne, Tetrahedron Lett., 2008, 49, 376-378.

56 H. Li, H.-R. Zhang, J. L. Petersen and K. K. Wang, J. Org. Chem., 2001, 66, 6662-6668; Y. Yang, J. L. Petersen and K. K. Wang, J. Org. Chem., 2003, 68, 5832-5837; Y. Yang, J. L. Petersen and K. Wang, J. Org. Chem., 2003, 68, 8545-8549.

57 S. Kitagaki, Y. Okumara and C. Mukai, Tetrahedron, 2006, 62, 10311-10320.

58 H. Cao, J. Flippen-Anderson and J. M. Cook, J. Am. Chem. Soc., 2003, 125, 3230-3231.

59 J. C. Martin, P. G. Gott, V. W. Goodlett and R. H. Hasek, J. Org. Chem., 1965, 30, 4175-4180; H. A. Bampfield and P. R. Brook, J. Chem. Soc., Chem. Commun., 1974, 171-172; H. A. Bampfield and P. R. Brook, J. Chem. Soc., Chem. Commun., 1974, 171-172; H. A. Bampfield, P. R. Brook and W. S. McDonald, J. Chem. Soc., 1975, 132-133; W. T. Brady, J. D. Stockon and A. D. Patel, J. Org. Chem., 1974, 39, 236-238; D. C. England and C. G. Krespan, J. Org. Chem., 1970, 35, 3322-3327; T. Minami, T. Okauchi, H. Matsuki, M. Nakamura, J. Ichikawa and M. Ishida, J. Org. Chem., 1996, 61, 8132-8140.

60 K. L. McCaleb and R. Halcomb, Org. Lett., 2000, 2, 2631-2634.

61 For general reviews on $[2+2]$-photocycloaddition reactions, see: S. A. Fleming, in Molecular and Supramolecular Photochemistry: Synthetic Organic Photochemistry, ed. A. G. Griesbeck and J. Mattay, Dekker, New York, 2005, vol. 12, pp. 141-160; P. Margaretha, in Molecular and Supramolecular Photochemistry: Synthetic Organic Photochemistry, ed. A. G. Griesbeck and J. Mattay, Dekker, New York, 2005, vol. 12, pp. 211-237.

62 S. W. Baldwin, in Organic Photochemistry, ed. A. Padwa, Dekker, New York, 1981, vol. 5, pp. 123-225; W. Oppolzer, Acc. Chem. Res., 1982, 15, 135; A. C. Weedon, in Synthetic Organic Photochemistry, ed. W. M. Horspool, Plenum, New York, 1984, pp. 61-144; D. I. Schuster, in Chemistry of enones, ed. S. Patai and Z. Rappoport, Wiley, New York, 1989, vol. 5, pp. 623-756; D. De Keukeleire and S.-L. He, Chem. Rev., 1993, 93, 359; CRC, Handbook of Organic Photochemistry and Photobiology, ed. W. M. Horspool and P.-S. Song, CRC Press, Boca Raton, FL, 1995, ch. 52 and 53; M. T. Crimmins, Chem. Rev., 1988, 88, 1453.

63 K. Wiesner, Tetrahedron, 1975, 31, 1655; S. L. Schreiber, Science, 1985, 227, 857; M. T. Crimmins, Chem. Rev., 1988, 88, 1453-1473; M. T. Crimmins and T. L. Reinhold, Organic Reactions, Wiley,
New York, 1993, vol. 44, p. 297; J. D. Winkler, C. M. Bowen and F. Liotta, Chem. Rev., 1995, 95, 2003-2020.

64 K. Wiesner, V. Musil and K. J. Wiesner, Tetrahedron Lett., 1968, 9, 5643-5646.

65 M. C. Pirrong and S. A. Thomson, J. Org. Chem., 1988, 53, 227-230.

66 P. E. Eaton, Tetrahedron Lett., 1964, 5, 3695-3698.

67 M. Tada and Y. Nieda, Bull. Chem. Soc. Jpn., 1988, 61, 1416-1418; G. L. Lange, M. G. Organ and M. Lee, Tetrahedron Lett., 1990, 31, 4689-4692; G. V. T. Swapna, A. B. Lakshmi, J. M. Rao and A. C. Kunwar, Tetrahedron, 1989, 45, 1777-1782; D. Andrew, D. J. Hastings, D. L. Oldroyd, A. Rudolph, A. C. Weedon, D. F. Wong and B. Zhang, Pure Appl. Chem., 1992, 64, 1327-1334.

68 J. F. Blount, Tetrahedron Lett., 1980, 21, 4413-4416; K. Wiesner, Tetrahedron, 1975, 31, 1655-1658; D. Becker, M. Nagler, Z. Harel and A. Gillon, J. Org. Chem., 1983, 48, 2584-2590.

69 Y.-H. Kang and J. L. Kice, Tetrahedron Lett., 1982, 23, 5373-5374; J. L. Kice and Y.-H. Kang, Tetrahedron, 1985, 41, 4739-4746.

70 D. J. Hastings and A. C. Weedon, J. Am. Chem. Soc., 1991, 113, 8525-8527; D. J. Hastings and A. C. Weedon, Tetrahedron Lett., 1991, 32, 4107-4110; D. J. Maradyn and A. C. Weedon, Tetrahedron Lett., 1994, 35, 8107-8110; D. Andrew, D. J. Hastings and A. C. Weedon, J. Am. Chem. Soc., 1994, 116, 10870-10882; D. J. Maradyn and A. C. Weedon, J. Am. Chem. Soc., 1995, 117, 5359-5360; D. Andrew and A. C. Weedon, J. Am. Chem. Soc., 1995, 117, 5647-5663.

71 D. J. Maradyn, L. K. Sydnes and A. C. Weedon, Tetrahedron Lett., 1993, 34, 2413-2416.

72 R. D. J. Froese, G. L. Lange and J. D. Goddard, J. Org. Chem., 1996, 61, 952-961.

73 E. M. Carreria, C. A. Hastings, M. S. Shepard, L. A. Yerkey and D. B. Millward, J. Am. Chem. Soc., 1994, 116, 6622-6630.

74 D. Becker, M. Nagler, Y. Sahali and N. Haddad, J. Org. Chem., 1991, 56, 4537-4543.

75 M. S. Shepard and E. M. Carreira, J. Am. Chem. Soc., 1997, 119, 2597-2605.

76 J. D. Winkler and J. R. Ragains, Org. Lett., 2006, 8, 4031-4033.

77 For reviews on the photochemistry of divinylmethanes, see: H. E. Zimmerman and D. Armesto, Chem. Rev., 1996, 96, 3065-3112; H. E. Zimmerman, Org. Photochem., 1991, 11, 1-36; H. E. Zimmerman, Acc. Chem. Res., 1982, 15, 312-317; S. S. Hixson and H. E. Zimmerman, Chem. Rev., 1973, 73, 531-551.

78 H. E. Zimmerman and R. E. Factor, Tetrahedron, 1981, 37, 125-141; P. Baeckström, Tetrahedron, 1978, 34, 3331-3335; P. Baeckström, J. Chem. Soc., Chem. Commun., 1976, 476-477; M. J. Bullivant and G. Pattenden, J. Chem. Soc., Perkin Trans. 1, 1976, 256-258.

79 H. E. Zimmerman, D. Armesto, M. G. Amezua, T. P. Gannett and R. P. Johnson, J. Am. Chem. Soc., 1979, 101, 6367-6383; H. E. Zimmerman and J. M. Cassel, J. Org. Chem., 1989, 54, 3800-3816.

80 K. Sugiyama and T. Tsuno, Chem. Express, 1992, 7, 929-932.

81 T. Tsuno and K. Sugiyama, Bull. Chem. Soc. Jpn., 1999, 72, 519-531.

82 T. Tsuno, H. Hoshino and K. Sugiyama, Tetrahedron Lett., 1997, 38, $1581-1584$.

83 T. Tsuno, H. Hoshino, R. Okuda and K. Sugiyama, Tetrahedron, 2001, 57, 4831-4840.

84 T. Tsuno and K. Sugiyama, Chem. Lett., 1991, 503-507; T. Tsuno and K. Sugiyama, Bull. Chem. Soc. Jpn., 1995, 68, 3175-3188; T. Tsuno, H. Hoshino and K. Sugiyama, Tetrahedron Lett., 1997, 38, 1581-1726.

85 T. Tsuno, M. Yoshida, T. Iwata and K. Sugiyama, Tetrahedron, 2002, 58, 7681-7689.

86 B. T. B. Hue, J. Dijkink, S. Kuiper, S. van Schaik, J. H. van Maarseveen and H. Hiemstra, Eur. J. Org. Chem., 2006, 127-137.

87 R. Miaou, S. G. Gramani and M. J. Lear, Tetrahedron Lett., 2009, 50, 1731-1733.

88 M. Lautens, W. Klute and W. Tam, Chem. Rev., 1996, 96, 49-92.

89 S. Saito, K. Hirayama, C. Kabuto and Y. Yamamoto, J. Am. Chem. Soc., 2000, 122, 10776-10780. 
90 F. F. Caserio, S. H. Parker, R. Piccolini and J. D. Roberts, J. Am. Chem. Soc., 1958, 80, 5507-5513.

91 A. T. Blomquist and J. A. Verdol, J. Am. Chem. Soc., 1956, 78, 109-112; W. T. Borden, I. L. Reich, L. A. Sharpe and R. B. Weinberg, J. Org. Chem., 1975, 40, 2438-2442.

92 E. Bustelo, C. Guérot, A. Hercouet, B. Carboni, L. Toupet and P. H. Dixneuf, J. Am. Chem. Soc., 2005, 127, 11582-11583.

93 T. Akiyama, K. Daidouji and K. Fuchibe, Org. Lett., 2003, 5, 3691-3693.

94 A. S. K. Hashmi and G. J. Hutchings, Angew. Chem., Int. Ed., 2006, 45, 7896-7936.

95 M. R. Luzung, P. Mauleón and F. D. Toste, J. Am. Chem. Soc., 2007, 129, 12402-12403.

96 Q. Shen and G. B. Hammond, J. Am. Chem. Soc., 2002, 124, $6534-6535$.
97 H. Cao, S. G. Van Ornum, J. Deschamps, J. Flippen-Anderson, F. Laib and J. M. Cook, J. Am. Chem. Soc., 2005, 127, 933-943. 98 F. Inagaki, S. Narita, T. Hasegawa, S. Kitagaki and C. Mukai, Angew. Chem., Int. Ed., 2009, 48, 2007-2011.

99 C. H. Oh and A. Kim, Synlett, 2008, 777-781.

100 C. Bruneau, Angew. Chem., Int. Ed., 2005, 44, 2328-2334; A. M. Echavarren and C. Nevado, Chem. Soc. Rev., 2004, 33, 431-436.

101 T. Matsuda, S. Kadowaki, T. Goya and M. Murakami, Synlett, 2006, 575-578

102 M. D. Clay and A. G. Fullis, Angew. Chem., Int. Ed., 2005, 44, 4039-4042; S. M. Kim, J. H. Park, Y. K. Kang and Y. K. Chung, Angew. Chem., Int. Ed., 2009, 48, 4532-4535.

103 X. Jiang, X. Cheng and S. Ma, Angew. Chem., Int. Ed., 2006, 45, 8009-8013. 